TITLE:

\title{
Urban density mapping of global megacities from polarimetric SAR images
}

$\operatorname{AUTHOR}(\mathrm{S})$ :

Susaki, Junichi; Kajimoto, Muneyoshi; Kishimoto, Masaaki

\section{CITATION:}

Susaki, Junichi ...[et al]. Urban density mapping of global megacities from polarimetric SAR images. Remote Sensing of Environment 2014, 155: $334-348$

ISSUE DATE:

2014-12

URL:

http://hdl.handle.net/2433/192762

RIGHT:

(C) 2014 Elsevier Inc:; This is not the published version. Please cite only the published version.; この論文は出版社版でありません。引用の際に は出版社版をご確認ご利用ください。 
5 a Associate Professor, Department of Civil and Earth Resources Engineering, Graduate School of Engineering, Kyoto

6 University, Kyoto, Japan

7 Postal address: C1-1-206 Kyotodaigakukatsura, Nishikyo-ku, Kyoto 615-8540, Japan

8 E-mail address: susaki.junichi.3r@kyoto-u.ac.jp; Tel. \& Fax: + 81-75-383-3300.

$9 \quad$ b NTT DOCOMO, INC., Tokyo, Japan

${ }^{\mathrm{c}}$ Department of Global Engineering, Faculty of Engineering, Kyoto University, Kyoto, Japan

Abstract-We propose an algorithm for estimating urban density from polarimetric synthetic aperture radar (SAR) images, and compare the urban density patterns of global megacities. SAR images are uniquely able to detect structural information of objects, but they are very sensitive to orientation angle. This issue has been an obstacle to applying SAR images to urban areas. Kajimoto and Susaki (2013b) proposed an algorithm to handle this issue. The effects of polarization orientation angle (POA) are removed by rotating the coherency matrix and then calculating the mean and standard deviation of scattering power by POA domain. The algorithm can estimate urban density from a single fully polarimetric SAR image but has the drawback that the generated urban density maps of multiple images are not comparable with each other because the algorithm generates a relative urban density valid only within the analyzed image. We therefore extend the method by calculating POA-domain statistics from all images of interest so that the generated maps can be compared. Estimated urban densities are assessed on two types of urban density generated from GIS data, building-to-land ratio and floor-area ratio. We demonstrate that the extended method can estimate urban density with reasonable accuracy. Finally, we generate two scattergrams of indices derived from urban density maps of global megacities. 
An analysis using the scattergrams indicates insightful information about the patterns of urban development.

We conclude that the proposed algorithm and the analysis using the obtained results are beneficial to understanding the conditions in megacities.

Index Terms - Urban density, megacities, polarimetric synthetic aperture radar, polarization orientation angle.

Mapping of human settlements is one of the most important applications of remote sensing. As the world population has increased, many megacities with populations exceeding one million have emerged, especially in Asia. Megacities such as Beijing, Bangkok, and Jakarta are still rapidly growing. Rapid growth of megacities in developing countries can cause severe urban problems, including problems related to traffic congestion, water supply, sewage disposal, air pollution, and housing. Before national or local governments can plan countermeasures against such urban problems, the areas of human settlement must be delineated. Population density should also be mapped at the district level to effectively determine budgets and improve the quality of urban life.

One traditional approach to mapping urban areas and density is to use census data to generate maps with the help of a geographic information system (GIS). However, the initial cost of collecting census data and converting them into digital data, and the ongoing cost of updating such data, are significant. This is true not only in developing countries, but also in developed countries. For example, in Japan, Zenrin Co. Ltd. is well known for selling detailed census data and manually updating this data. These data are sold commercially as Zmap Town II by local government organizations. For example, the Tokyo metropolitan area includes Chiba, Saitama, and Kanagawa prefectures and parts of Ibaraki prefecture. The area had a population of 37.6 million in an area of 14,000 km² in 2010 (Statistics Bureau, 2011). It costs approximately 300,000 USD to purchase the Zmap Town II data that includes the number of stories of buildings in the Tokyo metropolitan area (Zenrin, 2014). Because these data are so costly, most social science, civil engineering, and architecture researchers interested in urban areas have to find other sources of urban area data.

The estimation of population density in urban areas can be difficult because it requires an accurate population census. denotes building density. In this research, our motivation is to map urban density and urban areas for megacities 
throughout the world, thus promoting analysis and research on urban environments.

Remote sensing has the potential to map urban areas and density via several approaches. As daytime optical images, Landsat-series images have been widely used to monitor urban areas (Schneider, 2012; Zhu et al., 2012). Landsat has carried the Multispectral Scanner System (MSS), Thematic Mapper (TM), and Enhanced Thematic Mapper Plus (ETM+) devices. Because their basic designs are highly similar, long-term monitoring is possible. Bagan and Yamagata (2012) conducted an analysis of urban growth in the metropolitan Tokyo area by fusing long-term Landsat imagery and statistical data. High-temporal-resolution sensors, such as the Advanced Very High Resolution Radiometer (AVHRR) and Moderate Resolution Imaging Spectroradiometer (MODIS) have been also used for global mapping of urban areas (Friedl et al., 2002; Schneider et al., 2010). Nighttime optical sensors were used to extract urban areas by detecting nighttime illumination from urban areas. Defense Meteorological Satellite Programme-Operational Line Scanner (DMSP-OLS) provided such nighttime imagery, and urban maps generated using that imagery have been reported (Elvidge et al., 1997; Elvidge et al., 1998; Sutton, 2003). However, optical sensors have a critical drawback: they are sensitive to atmospheric conditions. For example, few clear optical images of Asian countries can be acquired during the monsoon season.

Synthetic aperture radar (SAR) and other microwave-based radar sensors are generally insensitive to atmospheric conditions, and interferometric SAR (InSAR) may be a useful approach to estimating heights for urban density mapping. Scattering mechanisms are very complex in urban areas due to multiple scattering by man-made structures (Margarit et al., 2010). Urban digital elevation models (DEM) estimated by InSAR are thus generally not accurate, but several approaches to improving accuracy have been presented (Thiele et al., 2007; Shabou et al., 2012). Permanent scatter InSAR (PSInSAR) (Ferretti et al., 2001) and SqueeSAR (Ferretti et al., 2011) generate DEM with very high accuracy (millimeter scale), even for urban areas (Ferretti et al., 2000; Stramondo et al., 2008; Perissin \& Wang, 2012; Chaussard et al., 2014). However, the major obstacle to implementing such techniques is that they require dozens of SAR images, making it hard to map many megacities.

Another feature of SAR is detection of structural information of surface targets. Fully polarimetric SAR (PolSAR) can provide data for four different combinations of horizontal (H)- and vertical (V)-polarization reception and transmission: HH, HV, VH, and VV. Three-component (Freeman \& Durden, 1998) and four-component decomposition algorithms (Yamaguchi et al., 2005; Yamaguchi et al., 2006) decompose multi-polarization data into three or four scattering components: surface, double-bounce, and volume scatterings are common to both algorithms, and helix 
scattering was added by the latter algorithm. Such analysis is quite different from when optical images are used.

This feature can be used to map urban density. Niu and Ban used PolSAR data to extract high- and low-density urban areas (Niu and Ban, 2012) where no density information was given for industrial, commercial, and construction areas. One obstacle to mapping using SAR data is the effect of polarization orientation angle (POA) (Kimura, 2008). The scattering received by SAR sensor is very sensitive to the POA of the target. This effect is more evident in urban areas than with vegetated land cover such as forests and agricultural areas. Kajimoto and Susaki (2013b) overcame this POA effect and succeeded in mapping urban density from only one PolSAR image of an area of interest. However, the method generates a relative density index that is applicable to only the analyzed image. The method is therefore not guaranteed to be applicable to all urban areas for comparing the status of urbanization of different megacities.

We extended the method proposed in Kajimoto and Susaki (2013b), and propose a method that estimates urban density from only one PolSAR image and enables comparison of urban densities of different cities. As described in Section 3, building density can be defined in several ways, such as building-to-land ratio and floor-area ratio. In this paper, the urban density estimated using PolSAR images is not defined in advance but rather assessed according to the kind of building density the estimated urban density is attributed to. Urban areas are defined as areas where artificial objects are dominant. The remainder of this paper is organized as follows: Section 2 describes the new method. Experimental results are reported in Section 3 and discussed in Section 4. Finally, we present our conclusions in Section 5.

\section{Methods}

\subsection{Outline of the Method}

Fig. 1 shows a flowchart of the proposed method, which uses fully polarimetric phase and amplitude data. First, POA is calculated, and four components with POA effect correction are generated. Next, urban areas are extracted using the method proposed by Kajimoto and Susaki (2013a). Finally, urban densities of multiple scenes are calculated. In this process, statistics (mean and standard deviation of scattering) are obtained by POA, as is homogeneous (or heterogeneous) status over the entire study area. 


\subsection{Polarimetric SAR Data}

The format of PolSAR data consists of a complex scattering matrix

$$
s=\left(\begin{array}{ll}
S_{H H} & S_{H V} \\
S_{V H} & S_{V V}
\end{array}\right)=\left(\begin{array}{ll}
a & c \\
c & b
\end{array}\right)
$$

Here, for simplicity, $S_{H V}$ and $S_{V H}$ are assumed to be equivalent, so the coherency matrix is given by

$$
\begin{gathered}
T=\left(\begin{array}{ccc}
T_{11} & T_{12} & T_{13} \\
T_{21} & T_{22} & T_{23} \\
T_{31} & T_{32} & T_{33}
\end{array}\right) \\
=\frac{1}{2}\left(\begin{array}{ccc}
|a+b|^{2} & (a+b)(a-b)^{*} & 2(a+b) c^{*} \\
(a-b)(a+b)^{*} & |a-b|^{2} & 2(a-b) c^{*} \\
2 c(a+b)^{*} & 2 c(a-b)^{*} & 4|c|^{2}
\end{array}\right),
\end{gathered}
$$

\subsection{Polarization Orientation Angle (POA)}

The polarization orientation angle (POA) estimates the azimuth angle of the target (Kimura, 2008). In this paper, the POA is denoted by $\phi$, which is not the typical notation for POA. We do this because we discuss the effect of the off-nadir angle difference in Section 4.4, and the off-nadir angle of radar is denoted by $\theta$ in this paper. $\phi$ is estimated as

The angle $\phi$ is determined by minimizing $T_{33}(\phi)$.

\subsection{Four-component Decomposition}

Four-component decomposition decomposes observed backscattering into four components calculated from the coherency matrix (Yamaguchi et al., 2005; Yamaguchi et al., 2006). Applying the four-component decomposition 
134

135

method to the full PolSAR data gives the surface scattering power $(P s)$, the double-bounce scattering power $(P d)$, the volume scattering power $(P v)$, and the helix scattering power $(P c)$.

Four components are sensitive to POA. Yamaguchi et al. (2011) proposed an algorithm that rotates the coherency matrix by the POA to reduce the dependence of the components on the relative azimuth angle. A rotation is applied to the coherency matrix:

$$
T(\phi)=\left(\begin{array}{lll}
T_{11}(\phi) & T_{12}(\phi) & T_{13}(\phi) \\
T_{21}(\phi) & T_{22}(\phi) & T_{23}(\phi) \\
T_{31}(\phi) & T_{32}(\phi) & T_{33}(\phi)
\end{array}\right)=\left[R_{p}(\phi)\right] T\left[R_{p}(\phi)\right]^{\dagger} .
$$

Here, $\dagger$ denotes complex conjugation and transposition, and $R_{p}(\phi)$ is the rotation matrix given by

$$
\left[R_{p}(\phi)\right]=\left(\begin{array}{ccc}
1 & 0 & 0 \\
0 & \cos 2 \phi & \sin 2 \phi \\
0 & -\sin 2 \phi & \cos 2 \phi
\end{array}\right)
$$

However, components remain dependent on the relative azimuth angle even after this correction (Iwasa \& Susaki, 2011), and removal of the remaining angular effects is a nontrivial problem.

\subsection{Urban Area Classification}

Urban areas are discriminated from other types of land cover (mountain, farmland, bare ground, and sea surface) by using the method proposed by Kajimoto and Susaki (2013a). Analysis using L-band PolSAR images indicated that POA-corrected Pv generated by four-component decomposition with Eq. (4) is less sensitive to POA than other POA-corrected components, but there is still a dependency on POA. Another difficulty is that the scattering intensity in non-orthogonal urban areas and that in orthogonal farmland is similar in some cases. Here, an "orthogonal” area denotes an area that has an almost $0^{\circ}$ POA. Therefore, in the first stage, POA-corrected Pv and total power (TP) data are used for 
classification. TP is derived as $T P=|a|^{2}+|b|^{2}+2|c|^{2}$. The combination of the two variables improves classification of land cover. In addition, pixels are categorized on POA as $\left(-7.5^{\circ}\right.$ to $\left.7.5^{\circ}\right),\left(-22.5^{\circ}\right.$ to $7.5^{\circ}, 7.5^{\circ}$ to $\left.22.5^{\circ}\right),\left(-37.5^{\circ}\right.$ to $22.5^{\circ}, 22.5^{\circ}$ to $\left.37.5^{\circ}\right)$, and $\left(-45.0^{\circ}\right.$ to $-37.5^{\circ}, 37.5^{\circ}$ to $\left.45.0^{\circ}\right)$. A different classification threshold is set for each category. In this classification, training data for urban areas and farmland in the study area are manually selected. Principal component analysis is applied to the training data, and the threshold on the first principal component for discriminating between urban areas and farmland is determined. The threshold is determined from the means and standard deviations of the first principal components of the two land cover types. When classifying multiple images, optimal thresholds for the study area of interest are automatically updated. The difference between the means of the two land covers is divided by the standard deviations, and the breakpoint is used as the threshold. The threshold is then applied to another study area, and an attempt is made to separate the urban areas from farmland. The difference is calculated between the urban gravity points in the new area and in the initial study area, and this difference is used to adjust the threshold. The updated threshold is again applied to the new study area, and the gravity point difference is calculated. Iteration of this process is terminated when the change in the threshold is within a predefined limit.

After the first stage of classification, both urban areas and mountainous areas are discriminated from the other three land covers (farmland, bare ground, and sea surface). Because most of the pixels of urban areas and mountainous areas overlap in Pv-TP space, the two land covers are not discriminated by using values of only scattering components. Therefore, in the second step, urban areas are discriminated from mountainous areas using POA randomness, rather than variance of POA. The procedure to count POA randomness is as follows. First, each pixel is labeled using one of five POA-based groups, $\left(-45^{\circ}\right.$ to $\left.25^{\circ}\right),\left(-25^{\circ}\right.$ to $\left.-5^{\circ}\right),\left(-5^{\circ}\right.$ to $\left.5^{\circ}\right),\left(5^{\circ}\right.$ to $\left.25^{\circ}\right)$, and $\left(25^{\circ}\right.$ to $\left.45^{\circ}\right)$. Next, a window is set around the pixel to be analyzed. Taking each window pixel in turn, the POA labels of the four neighboring pixels are compared with the label of the central pixel, defined as the reference pixel. If all four pixels have labels that are equal to the reference pixel's label or that differ by exactly one, the pixel is not counted. In all other cases, the pixel is counted. The number of pixels counted is then assigned to the reference pixel. Using this procedure, the pixel count is expected to be small in urban areas and large in mountainous areas. 
186

187

188

189

190

191

192

\subsection{Urban Density Estimation}

The method used to estimate urban density is based on the method proposed by Kajimoto and Susaki (2013b). It consists of two steps, the extraction of homogeneous-POA city districts and the normalization of scattering-power components in each POA space. They classified urban areas into homogeneous and heterogeneous areas because even if two pixels have nearly the same POA, their scattering intensities can be very different, especially in orthogonal building areas. After that, an index for urban density is calculated for each category of urban area, homogeneous or heterogeneous.

First, POA variance is calculated as follows:

$$
\operatorname{Var}(i, j)=\frac{1}{N_{m n}} \sum_{m} \sum_{n}\left(\phi(m, n)-\mu_{\phi}(i, j)\right)^{2}
$$

Here, $\operatorname{Var}(i, j)$ is the POA variance of the pixel $(i, j), N_{m n}$ is the pixel count in the local Lee sigma filtering window of the pixel $(i, j), \phi$ is the POA, $(m, n)$ indicates the location of pixels lying within the local window, and $\mu_{\phi}(i, j)$ is the average POA within the local window. This calculation is done for all pixels of an image. The POA type $H(i, j)$ of pixel $(i, j)$ is given by

$$
H(i, j)= \begin{cases}\text { HomoPOA } & (\operatorname{Var}(i, j)<\text { Threshold }) \\ \text { HeteroPOA } & (\operatorname{Var}(i, j)>\text { Threshold })\end{cases}
$$

The threshold in Eq. (7) is set by using training data. As a result, urban areas are classified as either homogeneous or heterogeneous.

The influence of POA can be removed by normalizing scattering-power components in each POA space. First, the whole POA space is divided into specific intervals. Then, in each POA interval the average and the standard deviation of each power component's scattering intensity in urban areas are calculated separately for homogeneous and heterogeneous POA areas. Finally, the power component's scattering intensity is normalized for all pixels in each POA interval. According to the results reported by Kajimoto and Susaki (2013b), we selected $P_{v^{+c}}$ as an optimal scattering to represent urban density. The normalized scattering intensity is expressed as follows: 


$$
T_{v+c}(i, j, k)=\frac{P_{v+c}(i, j, k, \phi, H)-\mu_{v+c}(\phi, H)}{\sigma_{v+c}(\phi, H)}
$$

$$
\sigma_{v+c}^{2}(\phi, H)=\frac{1}{N(\phi, H)} \sum_{i} \sum_{j} \sum_{k}\left(P_{v+c}(i, j, k, \phi, H)-\mu_{v+c}(\phi, H)\right)^{2} .
$$

Here, $T$ is the normalized scattering intensity, $P$ is the original scattering intensity, $\mu$ and $\sigma$ are, respectively, the average

and standard deviation of the scattering intensity, which are calculated separately for homogeneous POA and heterogeneous POA areas in each POA interval, $(i, j)$ indicates the location of the reference pixel, $k$ indicates the SAR image number, and $\phi$ is the POA interval to which the $(i, j)$ pixel belongs. Note that the average and standard deviation are calculated across all urban areas examined. When the average and standard deviation are calculated scene by scene, samples for specific POAs may be insufficient. This may lead to overcorrection or undercorrection of scattering power.

In addition, the average and standard deviation may reflect the statistics of the area of interest, but may not be common to other areas. Therefore, in this research, the average and standard deviation are calculated across all areas.

Finally, $T_{v+c}$ is normalized to the range $[0,1]$ using Eq. (11):

\section{Dataset}

$$
T_{v+c}^{\prime}(i, j, k)=\left\{\begin{array}{cl}
0 & \left(T_{v+c}(i, j, k)<-A\right) \\
\frac{T_{v+c}(i, j, k)+A}{2 A} & \left(-A \leq T_{v+c}(i, j, k) \leq A\right) . \\
1 & \left(T_{v+c}(i, j, k)>A\right)
\end{array}\right.
$$

Here, $A$ is a constant.

This study uses fully polarimetric Advanced Land Observing Satellite (ALOS)/Phased Array type L-band SAR (PALSAR) level 1.1 (L1.1) data. The images have slant-range coordinate data. Furthermore, ALOS/Advanced Visible 
and Near Infrared Radiometer type 2 (AVNIR-2) optical sensor data were used as a reference. It is known that L-band SAR observation has significant effects from Faraday rotation, a phenomenon by which the plane of polarization is rotated, especially in tropical regions. This experiment assumed negligible effects of Faraday rotation, because correction was successful. We have two categories of PALSAR data: data with a $21.5^{\circ}$ off-nadir angle against the center of the scene (Data A) and data with a $23.1^{\circ}$ off-nadir angle (Data B). Table 1 shows a listing of Data A and B, respectively.

Accurate information on urban density for Japanese cities was obtained from Zmap-TOWN II (ZENRIN) data, which are residential maps of Japan. Accurate urban density data were generated from Zmap-TOWN II (GIS) data, with reference to previous research (Tanaka, 2011). Two measures of urban density were defined: building-to-land ratio and floor-area ratio. First, building polygon data are intersected by a mesh. Buildings lying across the mesh border are divided into pieces by the border line. The mesh size was tentatively set to $20 \mathrm{~m} \times 20 \mathrm{~m}$, which approximately corresponds to the ground resolution of PALSAR after a multilooking process. Building density is calculated as follows:

$$
\begin{aligned}
& D_{\text {Building-to-Land }}(i, j, k)=\frac{\sum_{l=1}^{n} S_{I}(i, j, k)}{S_{\text {Land }}(i, j, k)} \\
& D_{\text {Floor }}(i, j, k)=\frac{\sum_{l=1}^{n} S_{l}(i, j, k) \times F_{l}(i, j, k)}{S_{\text {Land }}(i, j, k)} \quad(\boldsymbol{I} \in(i, j, k) \text { pixel }) .
\end{aligned}
$$

Here, $D$ is the estimated building density, $S$ is an area, and $F$ is a building floor. The pair $(i, j)$ is the location of the reference pixel, $k$ indicates the SAR image number, and $l$ denotes the $l$ th building included in the $(i, j)$ pixel. Finally, GIS images were co-registered to PALSAR images by manually selecting ground control points between the images. Coefficients recorded in the leader files of PALSAR data calculate latitude and longitude for each pixel. With these latitudes and longitudes, urban density maps were automatically converted to the WGS 1984 coordinate system with UTM (Universal Transverse Mercator) projection. In this research, the UTM image grid size was set to $25 \mathrm{~m}$.

For cities outside Japan, we used Open Street Map (2014). Shape files of building distributions were available for Munich and New York. We therefore generated building-to-land images for those two cities, and used them for assessment of the estimated urban densities. 
268

\section{Experiments}

In this study, Lee’s sigma filter is applied to PALSAR images as a speckle filter (Lee et al., 2009). The local window size for the filtering was set to $5 \times 5$. In the process of urban area extraction, a $3 \times 3$ boxcar filter was applied to the coherency matrix. The boxcar filter is effective in removing speckle noise but blurs an image quite substantially. However, in urban density estimation, preserving a target signature is a top priority, so Lee’s sigma filter with the smallest window size, $5 \times 5$, was selected.

In urban area extraction, we followed the thresholds used in Kajimoto and Susaki (2013a). The minimum change in the urban gravity point was set to $0.01 \mathrm{~dB}$ to terminate the optimization loop. For POA randomness calculations, the window size was set at $31 \times 31$ pixels, and the ratio between the pixel count and the total number of pixels in the window for discriminating between urban and mountainous areas was set to 0.35 . The threshold in Eq. (7) is $185.5^{\circ}$ square, following Kajimoto and Susaki (2013b). The procedure for obtaining this value was determined by considering orthogonal building areas in Tokyo and Sapporo images. After manually determining regions of interest (ROIs) of homogeneous orthogonal building areas in the Tokyo and Sapporo areas, the thresholds, which include 95\% of all pixels belonging to the ROIs, were $188^{\circ}$ square (Tokyo) and $183^{\circ}$ square (Sapporo). The average of the two study area thresholds, $185.5^{\circ}$, was used. Because application of this threshold value to the study areas was successful in the experiments, we did not change the value.

In urban density estimation, A in Eq. (11) was set to 3 to normalize $T$ in Eq. (8).

\subsection{Examination of Two Dataset Differences}

We first examined the differences between the two datasets: Data A and B. Figs. 2(a) and (b) show the relation between POA and TP of homogeneous and heterogeneous districts, respectively, in eight Japanese cities. Data A includes the Tokyo metropolitan area (hereafter, “Tokyo”), Kyoto, Nagoya, Sendai, and Kobe, and Data B includes Osaka, Sapporo, and Fukuoka. Fig. 2 indicates that there is a significant gap between the curves of Data A and those of Data B. To examine differences between the two datasets in specific areas, we used Data A and B of Tokyo and Sapporo (Table 1). Figs. 3(a) and (b) show the relation between POA and TP of homogeneous and heterogeneous districts in the two cities. Fig. 3 indicates that the relation is dependent not on the orbital difference (ascending or descending), but on off-nadir angle. Data B (23.1 ${ }^{\circ}$ off-nadir angle) are not reliable because the relations between peaks of the curves of Sapporo A and Tokyo D are inconsistent between homogeneous and heterogeneous districts (Figs. 3(a) and 3(b)); the 
296

297

298

299

300

301

302

303

304

305

306

307

308 peak of the curve of Sapporo A is higher than that of Tokyo D in Fig. 3(a), but this is not the case in Fig. 3(b). This may be due to the quality of the calibration. As a result, we decided to use only Data A for further analysis.

\subsection{Effect of Spatial Scale}

In this research, we used fully polarimetric PALSAR images whose ground range resolution is approximately $25 \mathrm{~m}$, and generated urban density maps by aggregating the results of each pixel. As expected, the accuracy obtained at smaller spatial scales (e.g. $1 \mathrm{~km}, 10 \mathrm{~km}$ ) is better than that obtained at larger scales (e.g. $10 \mathrm{~m}, 100 \mathrm{~m}$ ), but the results lose more information. We investigated the optimal spatial scale for maps in terms of accuracy and detail. For accuracy, we examined the correlation coefficients using GIS data at different spatial scales such as $100 \mathrm{~m}, 200 \mathrm{~m}$, and $300 \mathrm{~m}$, as shown in Fig. 4(a). For the map detail, we examined mutual information (Kullback-Leibler information or distance), expressed as

$$
D(P \| Q)=\sum_{i} P(i) \log \frac{P(i)}{Q(i)}
$$

Here, $D(\mathrm{P} \| \mathrm{Q})$ denotes Kullback-Leibler information, and $P$ and $Q$ are discrete probability distributions. In this experiment, we compared the distribution of urban density [0:1] at each spatial scale to that of a $50 \mathrm{~m}$ scale (Fig. 4(b)). The interval of urban density for calculating Eq. (13) was set to 0.01. This index represents how much detail is lost with a spatial scale change.

The greatest difficulty in assessing optimal spatial scale is how to combine the correlation coefficient and the mutual information, because the mutual information represents only the relative distance between two probabilistic distributions. Because it seems quite difficult to find a reasonable solution, we did not combine them, but we qualitatively assessed the optimal spatial resolution by referring to the two results. The spatial scale of a thematic map depends on the map purpose. In this research, we decided that the correlation coefficient should not be less than a certain threshold, which we set as 0.7 for all cities. Then, according to Fig. 4(a), the optimal spatial scale was selected as $300 \mathrm{~m}$. 


\subsection{Effect of Incident Angle of Radar}

Fig. 5 and Table 2 show the effect of incident angle difference on the accuracy of urban density estimation. Note that the results were obtained using slant-range coordinate (original coordinate) images, for ease in calculating the incident angle of each pixel. Because the off-nadir angle was $21.5^{\circ}$ against the scene center, the incident angle at the scene center was approximately $24.0^{\circ}$. The incident angle, $\theta$, was classified into three ranges: $\theta \leq 23.0^{\circ}, 23.5^{\circ} \leq \theta \leq 24.5^{\circ}$, and $\theta \geq$ $25.0^{\circ}$. Table 2 shows the results of correlation coefficient calculations for 300-m-resolution GIS images. It shows that there is a significant difference among the correlation coefficients of the three ranges.

One approach to correcting the incident angle effect is to divide the backscatter coefficient by $\cos \theta$ (Shimada et al., 2007). We corrected the original fully polarimetric data by multiplying them by the factor $(\cos 24.0 \% \cos \theta)$ and assessed the estimated urban densities with GIS images. As a result, the correlation coefficients became a little worse (by about 0.01) than those without incident angle correction. Although a significant effect of the incident angle difference was found, it may not be simple to remove it. This improvement is left as a future task for mapping urban densities from SAR images.

\subsection{Accuracy Assessment of Urban Density Estimation}

Figs. 6, 7, 8, and 9 show the respective results for Tokyo, Kyoto, Munich, and New York, two Japanese cities and two non-Japanese cities. We selected these Japanese cities because Tokyo is a highly dense city; Kyoto is relatively homogeneous in terms of building height due to building regulations. In Figs. 6 and 7, panel (a) shows the AVNIR-2 image, (b) and (e) show the estimated urban density from PALSAR images, (c) and (f) show the building-to-land ratio, and (d) and (g) show the floor-area ratio. In Figs. 8 and 9, panel (a) shows the AVNIR-2 image, (b) and (d) show the estimated urban density from PALSAR images, and (c) and (e) show the building-to-land ratio. Floor-area ratio data were not available for Munich or New York.

The effect of the mean and standard deviation (Eqs. (9) and (10)) on the final results was examined. In this study, we defined a calibration that calculates the mean and standard deviation over all images used for the analysis, following Eqs. (9) and (10). Figs. 10(a) and 11(a) show scattergrams of GIS data and the results before calibration, meaning that the mean and standard deviation used for Eq. (8) were calculated within the individual scene. In contrast, Figs. 10(b) and 11(b) show the scattergram of GIS data and the results after calibration. These results were obtained with a $300 \mathrm{~m}$ 
resolution. Table 3 shows the results of correlation coefficient calculations for $300 \mathrm{~m}$ resolution with using GIS images. On the whole, correlations with the building-to-land ratio are higher than those with the floor-area ratio. This means that building-to-land ratio is better than floor-area ratio for estimating urban density from PolSAR images..

This paper extends the method previously proposed in Kajimoto and Susaki (2013b) to application for multi-scenes. The technique calculates statistics for multiple images, and then applies them to all multi-scene images. While this improvement might seem small, it has two important aspects from a statistical viewpoint. The first is normalization of the data. The previously proposed method is based on correction of POA effects in backscattering and related components. For instance, when the POA interval is set to $1^{\circ}$, we take samples and calculate statistics (mean and standard deviation) for $-45^{\circ},-44^{\circ}, \ldots, 44^{\circ}, 45^{\circ}$ POA. The sum of volumetric and helix scatterings is normalized by Eq. (8). Assume that we separately generate two urban density maps for two images. Because the statistics of the two images are different, the values have different meanings. Thus, urban density maps generated in this way are not comparable. Calculating statistics based on multiple images, however, enables generation of comparable urban density maps.

Another improvement is the robustness required for generating urban densities from multi scene images. We need samples for all POAs, and, as above, when the interval of POA is set to $1^{\circ}$, we need samples at $-45^{\circ},-44^{\circ}, \ldots, 44^{\circ}, 45^{\circ}$ POA. Of course, some POAs have a small number or no samples for a given scene. POA statistics derived from a small number of samples are unstable. This instability has less effect when the method is applied to one scene for estimating urban density because the pixels affected are very limited in the scene. When statistics obtained from one scene are used to correct POA effects in another, however, the instability becomes significant. We thus need samples from all POAs to generate sufficiently stable statistics for POA effect correction. Even given a certain number of samples for a specific POA from one scene, the proposed method takes samples from multi-scenes to calculate statistics. The statistics from multi-scenes may not be optimal for any individual scene, but they are of use in reducing error caused by applying the statistics from one scene to another.

We examined the effect of the number of cities used to calculate statistics. The results became stable when the number of cities was around 10, and adding additional cities resulted in little improvement. This indicates that robust estimation of urban density in multiple images requires a certain number of images, but that robustness can be achieved when sufficient samples for each POA are obtained.

Here again, we discuss the implications of Table 3. The calibration of mean and standard deviation contributed to a slight improvement of correlation with the building-to-land ratio (by 0.026 for 7 cities), and with the floor-area ratio (by 
0.025 for 5 cities). Figs. 10 and 11 show that changes in the estimated urban density caused by calibration are significant for some data in Tokyo and Sendai. The correlation coefficients of some areas after calibration were worse than those before calibration. The average and standard deviation (Eqs. (9) and (10)) were calculated for each area before calibration, and thus they may be optimal for the area. However, an important point is that generated urban density maps are not comparable because of the normalization by using the statistics specific to the area. After calibration, the statistics common to all 17 areas were used to normalize. Because this normalization functioned to shift plots of each city to a common line (Figs. 10(a) to 10(b) and Figs. 11(a) to 11(b)), it contributed to overall improvement of the correlation. Because our objective is to compare the urban densities of global megacities, such normalization is necessary. In this context, in Table 3, the results of overall scenes are much more important than those of each scene. While the improvement indicated by correlation coefficients was small, we continued to apply the proposed method to global megacities.

\subsection{Estimation of Urban Density of Global Megacities}

The urban densities of megacities in ascending-mode Data A (Table 1) are shown in Fig. 12. The images correspond to the areas of $20 \mathrm{~km} \times 20 \mathrm{~km}$. In addition to the images, we extended the proposed method to extracting meaningful statistics in areas and districts. Two sizes of the urban area were set to $10 \mathrm{~km} \times 10 \mathrm{~km}$ and $20 \mathrm{~km} \times 20 \mathrm{~km}$. As for the districts, two sizes were set to $2.5 \mathrm{~km} \times 2.5 \mathrm{~km}$ and $5 \mathrm{~km} \times 5 \mathrm{~km}$, but the center of the window was common to all window sizes. These sizes were selected as follows. We first set a district of $2.5 \mathrm{~km} \times 2.5 \mathrm{~km}$, and this district was automatically determined by examining the highest mean urban densities within the window. This size was determined by examining the size of the highest urban density district in multiple images. The $20 \mathrm{~km}$ size for areas was determined by considering the area covered by PALSAR images. In polarimetric measurement mode, a PALSAR swath is 20 to 65 km (JAXA, 2006), and the images used in the experiment have approximately $30 \mathrm{~km}$ swaths. It is ideal that the whole of a city should be extracted and compared with those of other cities. However, it was found that parts of some cities were not observed in the PALSAR images. Therefore, we decided to limit the area to compare global megacities. Two different sizes for districts and areas were set because the scattergram depended on the size and the comparison between the results with two different sizes may indicate information about urban distribution patterns. The other area size, 10 $\mathrm{km} \times 10 \mathrm{~km}$, was determined by halving each dimension of $20 \mathrm{~km} \times 20 \mathrm{~km}$. In the same manner, if we halve the $2.5 \mathrm{~km}$ 
$\times 2.5 \mathrm{~km}$ size of the district we get $1.25 \mathrm{~km} \times 1.25 \mathrm{~km}$, which is too small to represent urban density. We use therefore use $5 \mathrm{~km} \times 5 \mathrm{~km}$, obtained by doubling each dimension of the $2.5 \mathrm{~km} \times 2.5 \mathrm{~km}$ area size.

In calculating the mean, aggregated urban densities were divided by the number of samples where those urban densities were more than 0 . Then, it was visually checked whether the highest urban density district is included in the automatically selected district. All results except the one for Kobe were acceptable. In the case of the Kobe image, parts of the Osaka area were included, and the automatically selected district belonged to them. The highest urban density district for Kobe was automatically detected by limiting the search area. Finally, the area that includes the pre-determined district and shows the highest mean urban densities was detected for each scene. Fig. 13 shows the relation between mean urban density in a district (for each, either $2.5 \mathrm{~km} \times 2.5 \mathrm{~km}$ or $5 \mathrm{~km} \times 5 \mathrm{~km}$ ) and the skew of urban density in a wider area $(10 \mathrm{~km} \times 10 \mathrm{~km}$ or $20 \mathrm{~km} \times 20 \mathrm{~km})$. Skew is a statistical measure of asymmetry of a distribution, defined as follows:

$$
\text { Skew }=E\left[\left(\frac{X-\mu}{\sigma}\right)^{3}\right]
$$

Here, $X$ is a random variable, and $\mu$ and $\sigma$ are the mean and standard deviation of $X$, respectively. When the distribution has strong symmetry, the absolute value of the skew is close to 0 .

Fig. 13 indicates several interesting findings. The vertical axis of Fig. 13 denotes homogeneity of urbanization, with larger values indicating more heterogeneously urbanized and developed cities in specific districts. The first finding is that cities such as Melbourne and Sydney show local heterogeneity because their skews are relatively high at both spatial scales $(10 \mathrm{~km} \times 10 \mathrm{~km}$ and $20 \mathrm{~km} \times 20 \mathrm{~km})$. This finding is supported by Figs. 12(d) and 13(j). The second finding is that the skew change indicates the degree of homogeneity. The skew of Ho Chi Minh City significantly increased between the $10 \mathrm{~km}$ and $20 \mathrm{~km}$ scales. This feature is unique to Ho Chi Minh City. It indicates that homogeneous areas with higher urban densities are distributed on a $10 \mathrm{~km}$ scale and that urban densities are significantly different between inside and outside the highly urbanized $10 \mathrm{~km} \times 10 \mathrm{~km}$ area. Such a homogeneous area can be found in the left area of Fig. 12(b). The white triangle shows the international airport in Ho Chi Minh City. On the other hand, Tokyo, Taipei, Tehran, and Kyoto have small skew change between the $10 \mathrm{~km}$ and $20 \mathrm{~km}$ scales. This means that the homogeneous urban areas are found in a $20 \mathrm{~km} \times 20 \mathrm{~km}$ area in these cities. 
The third finding is that a few cities can be classified into same categories having similar urban structure by considering both plots in two scattergrams: (1) Tokyo and Taipei, (2) Munich and Beijing, (3) Kyoto and Tehran, (4) Melbourne and Sydney, and (5) Sendai and New Delhi. These similarities are also seen in Figs. 6, 7, 8 and 12. The final finding is that Vientiane, the capital and the largest city in the Laos, is much less urbanized than other cities in terms of urban density on a district level and an area level. On the basis of the previous discussion, we can compare the status of different global megacities by using PolSAR images and the proposed method.

\section{Conclusions}

We extended an existing effective density estimation algorithm to allow application to various areas, while the existing one was limited to application to single areas. A normalized combination of the volume scattering power and the helix scattering power $\left(T_{v+c}\right)$ was used to calculate urban density. The mean and standard deviation used for the normalization were obtained by a calibration referring to all images to be analyzed. As a result of validation with GIS images, a small improvement was confirmed and the urban density estimated from a single PolSAR image has a significant correlation with the building-to-land ratio. We then applied this improved method to global megacities, and generated a two-dimensional scattergram of mean and skew of urban densities. This scattergram enabled international comparison of megacities in terms of urban structure, and indicated several findings. As a result, we found that the proposed method and such discussion based on the scattergram were very useful in obtaining knowledge about the status of megacities, especially when fundamental statistics are lacking for megacities of interest.

In this study, we used L-band PALSAR images because fully polarimetric PALSAR images were available for many megacities over the world, and because stable results of urban mapping using L-band PolSAR images have been reported (Kajimoto \& Susaki, 2013b). Satellite-borne X-band PolSAR images, such as those taken by TerraSAR-X, are now available, and the proposed method may be applied to such images. However, it may be expected that the obtained results will be different from those obtained as L-band images because the radar sensitivity of scatterers is dependent on wavelength. Because multiple scattering frequently occurs in urban areas, longer wavelength radar may be more appropriate for urban densities that have high correlation with building-to-land ratio. In future work, we will compare urban density maps generated from L-band PolSAR images with those generated from X-band PolSAR images. 
455

456

457

458

459

460

461

462

463

464

465

Acknowledgments

This research was supported by a Grant-in-Aid for Scientific Research (KAKENHI) for Young Scientists (B) (No. 22760393), and by a program of the Fourth Advanced Land Observing Satellite-2 Research Announcement, Japanese Aerospace Exploration Agency. Zmap-Town II (ZENRIN) was provided by the Center for Spatial Information Science, The University of Tokyo.

\section{References}

Bagan, H., \& Yamagata, Y. (2012) Landsat analysis of urban growth: How Tokyo became the world’s largest megacity during the last 40 years. Remote Sensing of Environment, 127, 210-222.

Chaussard, E., Wdowinski, S., Cabral-Cano, E., \& Amelung, F. (2014) Land subsidence in central Mexico detected by ALOS InSAR time-series, Remote Sensing of Environment, 140, 94-106.

Elvidge, C. D., Baugh, K. E., Kihn, E. A., Kroehl, H. W., \& Davis, E. R. (1997) Mapping City Lights with nighttime data from the DMSP operational linescan system. Photogrammetric Engineering \& Remote Sensing, 63, 727-734.

Elvidge, C. D., Baugh, K. E., Dietz, J. B., Bland, T., Sutton, P. C., \& Kroehl, H. W. (1998) Radiance calibration of DMSP-OLS low-light imaging data of human settlements. Remote Sensing of Environment, 68, 77-88.

Ferretti, A., Prati, C., \& Rocca, F. (2000). Non-linear subsidence rate estimation using permanent scatterers in differential SAR Interferometry. IEEE Transactions on Geoscience and Remote Sensing, 38, 2202-2212.

Ferretti, A., Prati, C. \& Rocca, F. (2001) Permanent scatterers in SAR interferometry. IEEE Transactions on Geoscience and Remote Sensing, 39, 8-20.

Ferretti, A., Fumagalli, A., Novali, F., Prati, C., Rocca, F. \&Rucci, A. (2011) A new algorithm for processing interferometric data-stacks: SqueeSAR. IEEE Transactions on Geoscience and Remote Sensing, 49, 3460-3470. 
Freeman, A. \& Durden, S. L. (1998) A three-component scattering model for polarimetric SAR data. IEEE Transactions on Geoscience and Remote Sensing, 36, 936-973.

485

Friedl, M. A., McIver, D. K., Hodges, J. C. F., Zhang, Z. Y., Muchoney, D., Strahler, A. H., Woodcock, C. E., Gopal, S.,

Schneider, A., Cooper, A., Baccini, A., Gao, F., Schaaf, C. (2002) Global land cover mapping from MODIS: algorithms and early results. Remote Sensing of Environment, 83, 287-302.

489

Iwasa, S. \& Susaki, J. (2011) Classification of building area using azimuth angle and density indices derived from polarimetric SAR. Proceedings of Joint Urban Remote Sensing Event, 269-272.

492

JAXA (2006), About ALOS - PALSAR. Available at http://www.eorc.jaxa.jp/ALOS/en/about/palsar.htm last accessed: Jul 14, 2014.

495

Kajimoto, M., \& Susaki, J. (2013b) Urban density estimation from polarimetric SAR images based on a POA correction method. IEEE Journal of Selected Topics in Applied Earth Observations and Remote Sensing, 6, 1418-1429.

Kimura, H. (2008) Radar polarization orientation shifts in built-up areas. IEEE Geoscience Remote Sensing Letters. 5, 217-221.

Lee, J. S., Wen, J. H., Ainsworth, T. L., Chen, K. S., \& Chen, A. J. (2009) Improved sigma filter for speckle filtering of 507

Margarit, G., Mallorquí, J. J., \& Pipia, L. (2010) Polarimetric characterization and temporal stability analysis of urban 
511 Niu, X., \& Ban, Y. (2012) An adaptive contextual SEM algorithm for urban land cover mapping using multitemporal 512 high-resolution polarimetric SAR data. IEEE Journal of Selected Topics in Applied Earth Observations and Remote 513 Sensing. 5, 1129-1139.

514

OpenStreetMap Data Extracts, Open Street Map (2014) Available at http://download.geofabrik.de/ last accessed: Jul 13, 2014.

517

Perissin, D. \& Wang, T. (2012) Time-series InSAR applications over urban areas in China. IEEE Journal of Selected Topics in Applied Earth Observations and Remote Sensing. 4, 92-100.

520

Schneider, A., Friedl, M. A., \& Potere, D. (2010) Mapping global urban areas using MODIS 500-m data: New methods and datasets based on 'urban ecoregions.’ Remote Sensing of Environment, 114, 1733-1746.

523

524

Shabou, A., Baselice, F., \& Ferraioli, G. (2012) Urban digital elevation model reconstruction using very high resolution multichannel InSAR data. IEEE Transactions on Geoscience and Remote Sensing, 50, 4748-4758.

Shimada, M., Isoguchi, O., Tadono, T., Higuchi, R. \& Isono, K. (2007) PALSAR CALVAL summary and update 2007. Proceedings of IEEE International Geoscience and Remote Sensing Symposium 2007, 3593-3596.

532

Statistics Bureau, Japan (2011), Population census. Available at http://www.stat.go.jp/english/data/kokusei/ index.htm 
536 Stramondo, S., Bozzano, F., Marra, F., Wegmuller, U., Cinti, F.R., Moro, M. \& Saroli, M. (2008) Subsidence induced 537 by urbanisation in the city of Rome detected by advanced InSAR technique and geotechnical investigations, Remote 538 Sensing of Environment, 112, 3160-3172.

Sutton, P. C. (2003) A scale-adjusted measure of “Urban sprawl” using nighttime satellite imagery. Remote Sensing of 541 Environment, 86, 353-369.

542

Tanaka, K. (2011) The Land Institute of Japan, 2011. Formulation of urban density indices by using geospatial information: A case of Tokyo Metropolitan Area. Tokyo: The Land Institute of Japan, pp. 1-49, Japanese.

Thiele, A., Cadario, E., Schulz, K., Thönnessen, U., \& Soergel, U. (2007) Building recognition from multi-aspect high-resolution InSAR data in urban areas. IEEE Transactions on Geoscience and Remote Sensing, 45, 3583-3593.

Yamaguchi, Y., Moriyama, T., Ishido, M., \& Yamada, H. (2005) Four-component scattering model for polarimetric SAR image decomposition. IEEE Transactions on Geoscience and Remote Sensing, 43 1699-1706.

Yamaguchi, Y., Yajima, Y., \& Yamada, H. (2006) A four-component decomposition of POLSAR images based on the coherency matrix. IEEE Geoscience Remote Sensing Letters, 3, 292-296.

Yamaguchi, Y., Sato, A., Boerner, W., Sato, R., \& Yamada, H. (2011) Four-component scattering power decomposition with rotation of coherency matrix. IEEE Transactions on Geoscience and Remote Sensing, 49, 2251-2258.

557

Zenrin, Co. Ltd. (2014) Zmap Town II. Available at http:// www.zenrin.co.jp/product/gis/zmap/zmaptown.html (in Japanese) lass accessed: Jan 5, 2014.

Zhu, Z., Woodcock, C. E., Rogan, J., \& Kellndorfer, J. (2012) Assessment of spectral, polarimetric, temporal, and spatial dimensions for urban and peri-urban land cover classification using Landsat and SAR data. Remote Sensing of 
565

566

567

568

569

570

571

572

573

574

576

577

578

579

580

581

582

583

584

585

586

587

588

589

590
List of Figure Captions

Fig. 1. Flow of the proposed method

Fig. 2. Average TP of PALSAR images plotted against POA. (a) Average TP for homogeneous POA areas, and (b) average TP for heterogeneous POA areas. Data A with $21.5^{\circ}$ off-nadir angle against scene center includes Tokyo, Kyoto, Sendai, Nagoya, and Kobe. Data B with $23.1^{\circ}$ off-nadir angle includes Osaka, Fukuoka, and Sapporo.

Fig. 3. Average TP of PALSAR images (Sapporo and Tokyo) plotted against POA. Each city has two images with different off-nadir angle. (a) Average TP for homogeneous POA areas, and (b) average TP for heterogeneous POA areas. “ $A$ ” denotes ascending mode of observation, and “ $D$ ” denotes descending mode of observation.

Fig. 4. Effect of spatial scale on the results. (a) Correlation coefficient of estimated urban density between SAR data and GIS data, (b) mutual information (Kullback-Leibler information) compared to the data at a $50 \mathrm{~m}$ spatial scale.

Fig. 5. Effect of incident angle $\theta$ difference to the accuracy of urban density estimation. (a) Results in case when $\theta \leq$ $23.0^{\circ}$, (b) $23.5^{\circ} \leq \theta \leq 24.5^{\circ}$, and (c) $\theta \geq 25.0^{\circ}$.

Fig. 6. Results of urban density estimation for Tokyo. (a) AVNIR-2 image observed on January 11, 2007 (R:G:B = band 3:4:2), (b)(e) estimated urban density, (c)(f) building-to-land ratio, and (d)(g) floor area ratio. (b), (c), and (d) were original data, and aggregated into images with $300 \mathrm{~m}$ mesh size (e), (f), and (g), respectively.

Fig. 7. Results of urban density estimation for Kyoto. See Fig. 4 for a description of each panel. The AVNIR-2 image was observed on May 15, 2008. Note that some urban areas in (a) are not included in (c) and (d), because (c) and (d) have only data from inside Kyoto. 
591

592

593

594

595

596

597

598

599

600

601

602

603

604

605

606

607

608

609

610

611

Fig. 8. Results of urban density estimation for Munich. (a) AVNIR-2 image observed on September 22, 2010 (R:G:B = band 3:4:2), (b)(d) estimated urban density, and (c)(e) building-to-land ratio. (b) and (c) were original data, and aggregated into images with 300 m mesh size (d) and (e), respectively.

Fig. 9. Results of urban density estimation for New York. See Fig. 7 for a description of each panel. The AVNIR-2 image was observed on November 3, 2010.

Fig. 10. Effect of calibration to the assessment of the estimated urban density with building-to-land ratio. (a) Scattergram of GIS data and the results before calibration, in which the mean and standard deviation used for Eq. (8) were calculated within the individual scene. (b) Scattergram of GIS data and the results after calibration, in which the mean and standard deviation were calculated using Eqs. (9) and (10), respectively.

Fig. 11. Effect of calibration to the assessment of the estimated urban density with floor area ratio. See Fig. 9 for a description of each panel.

Fig. 12. Results of urban density estimation. (a) Beijing, (b) Ho Chi Minh, (c) Kobe, (d) Melbourne, (e) Nagoya, (f) New Delhi, (g) Sendai, (h) Shanghai, (i) Singapore, (j) Sydney, (k) Taipei, (l) Tehran, and (m) Vientiane.

Fig. 13. Scattergram of indices derived from estimated urban densities. (a) Relation between the highest mean in a 2.5 $\times 2.5 \mathrm{~km}$ district and the skew of urban density in a $10 \times 10 \mathrm{~km}$ area, and (b) relation between the highest mean in a 5 $\times 5 \mathrm{~km}$ district and the skew of urban density in a $20 \times 20 \mathrm{~km}$ area. 


\section{Elsevier Editorial System(tm) for Remote Sensing of Environment Manuscript Draft}

Manuscript Number: RSE-D-14-00133R3

Title: Urban Density Mapping of Global Megacities from Polarimetric SAR Images

Article Type: Original Research Paper

Keywords: Urban density; megacities; polarimetric synthetic aperture radar; polarization orientation angle

Corresponding Author: Dr. Junichi Susaki, Ph.D.

Corresponding Author's Institution: Kyoto University

First Author: Junichi Susaki, Ph.D.

Order of Authors: Junichi Susaki, Ph.D.; Muneyoshi Kajimoto, Master of Engineering; Masaaki Kishimoto, Bachelor of Engineering 


\section{Responses to comments by the Reviewers}

We are grateful to the Editor and the anonymous reviewers for their valuable comments. Below, we respond to the comment raised by the reviewers.

From: $\quad$ "Remote Sensing of Environment"<rse@ umn.edu>

To: $\quad$ susaki.junichi.3r@ kyoto-u.ac.jp

Date: $\quad 21$ Aug 2014 18:50:12 +0100

Subject: RSE-D-14-00133R2

Ref.: RSE-D-14-00133R2

Urban Density Mapping of Global Megacities from Polarimetric SAR Images

Dear Dr. Susaki,

Review of your revised paper follows. It is positive but suggests obtaining help with the English.

When you submit your revised paper, please provide a summary of the changes you have made and your responses to the review comments and recommendations. I will look forward to receiving your revised manuscript.

To submit a revision, go to http://ees.elsevier.com/rse/ and log in as an Author. You will see a menu item called "Submission Needing Revision." You will find your submission record there. Please remove any items that have changed or are no longer needed before uploading your revised manuscript.

Please upload your original files, not PDF files, as the publisher is not able to work with pdf files. Also, UPLOAD YOUR HIGH-RESOLUTION FIGURE FILES. Label each figure file in the DESCRIPTION box on the upload screen as "Figure 1, Figure 2, etc." ?If you have any problems or questions when uploading your revised manuscript, please contact Betty Schiefelbein at: rse@umn.edu.

PLEASE NOTE: The journal would like to enrich online articles by visualising and providing geographical details described in Remote Sensing of Environment articles. For this purpose, corresponding KML (GoogleMaps) files can be uploaded in our online submission system. Submitted KML files will be published with your online article on ScienceDirect. Elsevier will 
generate maps from the KML files and include them in the online article.

Please note that this journal offers a new, free service called AudioSlides: brief, webcast-style presentations that are shown next to published articles on ScienceDirect (see also http://www.elsevier.com/audioslides). If your paper is accepted for publication, you will automatically receive an invitation to create an AudioSlides presentation.

Sincerely,

Marvin Bauer

Editor-in-Chief

Remote Sensing of Environment

Reviewers' comments:

Reviewer \#3:

The paper has been revised according to my requests. I am glad the authors followed my suggestions to try and improve their paper.

At this point I only suggest that all the text, and especially the new one, should be revised for the English. As an example, the new paragraph on page 7 has multiple issues: " fist component threshold" should be "a threshold on the first principal component", "threshold optimal" should be "optimal threshold", "in proportion to the standard deviation" should by "by the standard deviation", and so on).

Like the previous manuscript, we asked an English proof company to edit the manuscript again. We hope you will be satisfied with the revision. 


\section{Highlights}

- We estimated urban areas and density from a single polarimetric SAR image.

- We calculated statistics from images to reduce orientation angle effects.

- The estimated urban density has a high correlation with building-to-land ratio.

- We compared the urban density patterns of global megacities.

- Analysis using urban density maps indicates the patterns of urban development. 


\title{
Urban Density Mapping of Global Megacities from
}

\section{Polarimetric SAR Images}

\author{
Junichi Susaki $^{\mathrm{a},{ }^{*}}$, Muneyoshi Kajimoto ${ }^{\mathrm{b}}$ and Masaaki Kishimoto ${ }^{\mathrm{c}}$ \\ ${ }^{a}$ Associate Professor, Department of Civil and Earth Resources Engineering, Graduate School of Engineering, Kyoto \\ University, Kyoto, Japan \\ Postal address: C1-1-206 Kyotodaigakukatsura, Nishikyo-ku, Kyoto 615-8540, Japan \\ E-mail address: susaki.junichi.3r@kyoto-u.ac.jp; Tel. \& Fax: + 81-75-383-3300. \\ ${ }^{\mathrm{b}}$ NTT DOCOMO, Co. Ltd., Tokyo, Japan \\ ${ }^{c}$ Department of Global Engineering, Faculty of Engineering, Kyoto University, Kyoto, Japan
}

Abstract -We propose an algorithm for estimating urban density from polarimetric synthetic aperture radar (SAR) images, and compare the urban density patterns of global megacities. SAR images are uniquely able to detect structural information of objects, but they are very sensitive to orientation angle. This issue has been an obstacle to applying SAR images to urban areas. Kajimoto and Susaki (2013b) proposed an algorithm to handle this issue. The effects of polarization orientation angle (POA) are removed by rotating the coherency matrix and then calculating the mean and standard deviation of scattering power by POA domain. The algorithm can estimate urban density from a single fully polarimetric SAR image but has the drawback that the generated urban density maps of multiple images are not comparable with each other because the algorithm generates a relative urban density valid only within the analyzed image. We therefore extend the method by calculating POA-domain statistics from all images of interest so that the generated maps can be compared. Estimated urban densities are assessed on two types of urban density generated from GIS data, building-to-land ratio and floor-area ratio. We demonstrate that the extended method can estimate urban density with reasonable accuracy. Finally, we generate two scattergrams of indices derived from urban density maps of global megacities. 
An analysis using the scattergrams indicates insightful information about the patterns of urban development. We conclude that the proposed algorithm and the analysis using the obtained results are beneficial to understanding the conditions in megacities.

\section{Index Terms - Urban density, megacities, polarimetric synthetic aperture radar, polarization orientation angle.}

\section{Introduction}

Mapping of human settlements is one of the most important applications of remote sensing. As the world population has increased, many megacities with populations exceeding one million have emerged, especially in Asia. Megacities such as Beijing, Bangkok, and Jakarta are still rapidly growing. Rapid growth of megacities in developing countries can cause severe urban problems, including problems related to traffic congestion, water supply, sewage disposal, air pollution, and housing. Before national or local governments can plan countermeasures against such urban problems, the areas of human settlement must be delineated. Population density should also be mapped at the district level to effectively determine budgets and improve the quality of urban life.

One traditional approach to mapping urban areas and density is to use census data to generate maps with the help of a geographic information system (GIS). However, the initial cost of collecting census data and converting them into digital data, and the ongoing cost of updating such data, are significant. This is true not only in developing countries, but also in developed countries. For example, in Japan, Zenrin Co. Ltd. is well known for selling detailed census data and manually updating this data. These data are sold commercially as Zmap Town II by local government organizations. For example, the Tokyo metropolitan area includes Chiba, Saitama, and Kanagawa prefectures and parts of Ibaraki prefecture. The area had a population of 37.6 million in an area of $14,000 \mathrm{~km}^{2}$ in 2010 (Statistics Bureau, 2011). It costs approximately 300,000 USD to purchase the Zmap Town II data that includes the number of stories of buildings in the Tokyo metropolitan area (Zenrin, 2014). Because these data are so costly, most social science, civil engineering, and architecture researchers interested in urban areas have to find other sources of urban area data.

The estimation of population density in urban areas can be difficult because it requires an accurate population census. Building density can be used as an alternative index to reflect the activities in urban areas. Hereinafter, urban density denotes building density. In this research, our motivation is to map urban density and urban areas for megacities 
throughout the world, thus promoting analysis and research on urban environments.

Remote sensing has the potential to map urban areas and density via several approaches. As daytime optical images, Landsat-series images have been widely used to monitor urban areas (Schneider, 2012; Zhu et al., 2012). Landsat has carried the Multispectral Scanner System (MSS), Thematic Mapper (TM), and Enhanced Thematic Mapper Plus (ETM+) devices. Because their basic designs are highly similar, long-term monitoring is possible. Bagan and Yamagata (2012) conducted an analysis of urban growth in the metropolitan Tokyo area by fusing long-term Landsat imagery and statistical data. High-temporal-resolution sensors, such as the Advanced Very High Resolution Radiometer (AVHRR) and Moderate Resolution Imaging Spectroradiometer (MODIS) have been also used for global mapping of urban areas (Friedl et al., 2002; Schneider et al., 2010). Nighttime optical sensors were used to extract urban areas by detecting nighttime illumination from urban areas. Defense Meteorological Satellite Programme-Operational Line Scanner (DMSP-OLS) provided such nighttime imagery, and urban maps generated using that imagery have been reported (Elvidge et al., 1997; Elvidge et al., 1998; Sutton, 2003). However, optical sensors have a critical drawback: they are sensitive to atmospheric conditions. For example, few clear optical images of Asian countries can be acquired during the monsoon season.

Synthetic aperture radar (SAR) and other microwave-based radar sensors are generally insensitive to atmospheric conditions, and interferometric SAR (InSAR) may be a useful approach to estimating heights for urban density mapping. Scattering mechanisms are very complex in urban areas due to multiple scattering by man-made structures (Margarit et al., 2010). Urban digital elevation models (DEM) estimated by InSAR are thus generally not accurate, but several approaches to improving accuracy have been presented (Thiele et al., 2007; Shabou et al., 2012). Permanent scatter InSAR (PSInSAR) (Ferretti et al., 2001) and SqueeSAR (Ferretti et al., 2011) generate DEM with very high accuracy (millimeter scale), even for urban areas (Ferretti et al., 2000; Stramondo et al., 2008; Perissin \& Wang, 2012; Chaussard et al., 2014). However, the major obstacle to implementing such techniques is that they require dozens of SAR images, making it hard to map many megacities.

Another feature of SAR is detection of structural information of surface targets. Fully polarimetric SAR (PolSAR) can provide data for four different combinations of horizontal $(\mathrm{H})$ - and vertical (V)-polarization reception and transmission: HH, HV, VH, and VV. Three-component (Freeman \& Durden, 1998) and four-component decomposition algorithms (Yamaguchi et al., 2005; Yamaguchi et al., 2006) decompose multi-polarization data into three or four scattering components: surface, double-bounce, and volume scatterings are common to both algorithms, and helix 
scattering was added by the latter algorithm. Such analysis is quite different from when optical images are used.

This feature can be used to map urban density. Niu and Ban used PolSAR data to extract high- and low-density urban areas (Niu and Ban, 2012) where no density information was given for industrial, commercial, and construction areas. One obstacle to mapping using SAR data is the effect of polarization orientation angle (POA) (Kimura, 2008). The scattering received by SAR sensor is very sensitive to the POA of the target. This effect is more evident in urban areas than with vegetated land cover such as forests and agricultural areas. Kajimoto and Susaki (2013b) overcame this POA effect and succeeded in mapping urban density from only one PolSAR image of an area of interest. However, the method generates a relative density index that is applicable to only the analyzed image. The method is therefore not guaranteed to be applicable to all urban areas for comparing the status of urbanization of different megacities.

We extended the method proposed in Kajimoto and Susaki (2013b), and propose a method that estimates urban density from only one PolSAR image and enables comparison of urban densities of different cities. As described in Section 3, building density can be defined in several ways, such as building-to-land ratio and floor-area ratio. In this paper, the urban density estimated using PolSAR images is not defined in advance but rather assessed according to the kind of building density the estimated urban density is attributed to. Urban areas are defined as areas where artificial objects are dominant. The remainder of this paper is organized as follows: Section 2 describes the new method. Experimental results are reported in Section 3 and discussed in Section 4. Finally, we present our conclusions in Section 5.

\section{Methods}

\subsection{Outline of the Method}

Fig. 1 shows a flowchart of the proposed method, which uses fully polarimetric phase and amplitude data. First, POA is calculated, and four components with POA effect correction are generated. Next, urban areas are extracted using the method proposed by Kajimoto and Susaki (2013a). Finally, urban densities of multiple scenes are calculated. In this process, statistics (mean and standard deviation of scattering) are obtained by POA, as is homogeneous (or heterogeneous) status over the entire study area. 
107

108

109

110

111

112

113

114

115

116

117

118

119

120

\subsection{Polarimetric SAR Data}

The format of PolSAR data consists of a complex scattering matrix

$$
s=\left(\begin{array}{ll}
S_{H H} & S_{H V} \\
S_{V H} & S_{V V}
\end{array}\right)=\left(\begin{array}{ll}
a & c \\
c & b
\end{array}\right) .
$$

Here, for simplicity, $S_{H V}$ and $S_{V H}$ are assumed to be equivalent, so the coherency matrix is given by

$$
\begin{gathered}
T=\left(\begin{array}{ccc}
T_{11} & T_{12} & T_{13} \\
T_{21} & T_{22} & T_{23} \\
T_{31} & T_{32} & T_{33}
\end{array}\right) \\
=\frac{1}{2}\left(\begin{array}{ccc}
|a+b|^{2} & (a+b)(a-b)^{*} & 2(a+b) c^{*} \\
(a-b)(a+b)^{*} & |a-b|^{2} & 2(a-b) c^{*} \\
2 c(a+b)^{*} & 2 c(a-b)^{*} & 4|c|^{2}
\end{array}\right) .
\end{gathered}
$$

\subsection{Polarization Orientation Angle (POA)}

The polarization orientation angle (POA) estimates the azimuth angle of the target (Kimura, 2008). In this paper, the POA is denoted by $\phi$, which is not the typical notation for POA. We do this because we discuss the effect of the off-nadir angle difference in Section 4.4, and the off-nadir angle of radar is denoted by $\theta$ in this paper. $\phi$ is estimated as

The angle $\phi$ is determined by minimizing $T_{33}(\phi)$.

$$
\phi=\frac{1}{4} \tan ^{-1} \frac{2 \operatorname{Re}\left(T_{23}\right)}{T_{22}-T_{33}},\left(-\frac{\pi}{4} \leq \phi \leq \frac{\pi}{4}\right) .
$$

\subsection{Four-component Decomposition}

Four-component decomposition decomposes observed backscattering into four components calculated from the coherency matrix (Yamaguchi et al., 2005; Yamaguchi et al., 2006). Applying the four-component decomposition 
method to the full PolSAR data gives the surface scattering power $(P s)$, the double-bounce scattering power $(P d)$, the volume scattering power $(P v)$, and the helix scattering power $(P c)$.

Four components are sensitive to POA. Yamaguchi et al. (2011) proposed an algorithm that rotates the coherency matrix by the POA to reduce the dependence of the components on the relative azimuth angle. A rotation is applied to the coherency matrix:

$$
T(\phi)=\left(\begin{array}{lll}
T_{11}(\phi) & T_{12}(\phi) & T_{13}(\phi) \\
T_{21}(\phi) & T_{22}(\phi) & T_{23}(\phi) \\
T_{31}(\phi) & T_{32}(\phi) & T_{33}(\phi)
\end{array}\right)=\left[R_{p}(\phi)\right][T]\left[R_{p}(\phi)\right]^{\dagger} .
$$

Here, $\uparrow$ denotes complex conjugation and transposition, and $R_{p}(\phi)$ is the rotation matrix given by

$$
\left[R_{p}(\phi)\right]=\left(\begin{array}{ccc}
1 & 0 & 0 \\
0 & \cos 2 \phi & \sin 2 \phi \\
0 & -\sin 2 \phi & \cos 2 \phi
\end{array}\right)
$$

However, components remain dependent on the relative azimuth angle even after this correction (Iwasa \& Susaki, 2011), and removal of the remaining angular effects is a nontrivial problem.

\subsection{Urban Area Classification}

Urban areas are discriminated from other types of land cover (mountain, farmland, bare ground, and sea surface) by using the method proposed by Kajimoto and Susaki (2013a). Analysis using L-band PolSAR images indicated that POA-corrected Pv generated by four-component decomposition with Eq. (4) is less sensitive to POA than other POA-corrected components, but there is still a dependency on POA. Another difficulty is that the scattering intensity in non-orthogonal urban areas and that in orthogonal farmland is similar in some cases. Here, an "orthogonal" area denotes an area that has an almost $0^{\circ} \mathrm{POA}$. Therefore, in the first stage, POA-corrected $\mathrm{Pv}$ and total power (TP) data are used for 
classification. TP is derived as $T P=|a|^{2}+|b|^{2}+2|c|^{2}$. The combination of the two variables improves classification of land cover. In addition, pixels are categorized on POA as $\left(-7.5^{\circ}\right.$ to $\left.7.5^{\circ}\right),\left(-22.5^{\circ}\right.$ to $7.5^{\circ}, 7.5^{\circ}$ to $\left.22.5^{\circ}\right),\left(-37.5^{\circ}\right.$ to $-22.5^{\circ}, 22.5^{\circ}$ to $\left.37.5^{\circ}\right)$, and $\left(-45.0^{\circ}\right.$ to $-37.5^{\circ}, 37.5^{\circ}$ to $\left.45.0^{\circ}\right)$. A different classification threshold is set for each category.

In this classification, training data for urban areas and farmland in the study area are manually selected. Principal component analysis is applied to the training data, and the threshold on the first principal component for discriminating between urban areas and farmland is determined. The threshold is determined from the means and standard deviations of the first principal components of the two land cover types. When classifying multiple images, optimal thresholds for the study area of interest are automatically updated. The difference between the means of the two land covers is divided by the standard deviations, and the breakpoint is used as the threshold. The threshold is then applied to another study area, and an attempt is made to separate the urban areas from farmland. The difference is calculated between the urban gravity points in the new area and in the initial study area, and this difference is used to adjust the threshold. The updated threshold is again applied to the new study area, and the gravity point difference is calculated. Iteration of this process is terminated when the change in the threshold is within a predefined limit.

After the first stage of classification, both urban areas and mountainous areas are discriminated from the other three land covers (farmland, bare ground, and sea surface). Because most of the pixels of urban areas and mountainous areas overlap in Pv-TP space, the two land covers are not discriminated by using values of only scattering components. Therefore, in the second step, urban areas are discriminated from mountainous areas using POA randomness, rather than variance of POA. The procedure to count POA randomness is as follows. First, each pixel is labeled using one of five POA-based groups, $\left(-45^{\circ}\right.$ to $\left.25^{\circ}\right),\left(-25^{\circ}\right.$ to $\left.-5^{\circ}\right),\left(-5^{\circ}\right.$ to $\left.5^{\circ}\right),\left(5^{\circ}\right.$ to $\left.25^{\circ}\right)$, and $\left(25^{\circ}\right.$ to $\left.45^{\circ}\right)$. Next, a window is set around the pixel to be analyzed. Taking each window pixel in turn, the POA labels of the four neighboring pixels are compared with the label of the central pixel, defined as the reference pixel. If all four pixels have labels that are equal to the reference pixel's label or that differ by exactly one, the pixel is not counted. In all other cases, the pixel is counted. The number of pixels counted is then assigned to the reference pixel. Using this procedure, the pixel count is expected to be small in urban areas and large in mountainous areas. 
187

\subsection{Urban Density Estimation}

The method used to estimate urban density is based on the method proposed by Kajimoto and Susaki (2013b). It consists of two steps, the extraction of homogeneous-POA city districts and the normalization of scattering-power components in each POA space. They classified urban areas into homogeneous and heterogeneous areas because even if two pixels have nearly the same POA, their scattering intensities can be very different, especially in orthogonal building areas. After that, an index for urban density is calculated for each category of urban area, homogeneous or heterogeneous.

First, POA variance is calculated as follows:

$$
\operatorname{Var}(i, j)=\frac{1}{N_{m n}} \sum_{m} \sum_{n}\left(\phi(m, n)-\mu_{\phi}(i, j)\right)^{2} .
$$

Here, $\operatorname{Var}(i, j)$ is the POA variance of the pixel $(i, j), N_{m n}$ is the pixel count in the local Lee sigma filtering window of the pixel $(i, j), \phi$ is the POA, $(m, n)$ indicates the location of pixels lying within the local window, and $\mu_{\phi}(i, j)$ is the average POA within the local window. This calculation is done for all pixels of an image. The POA type $H(i, j)$ of pixel $(i, j)$ is given by

$$
H(i, j)=\left\{\begin{aligned}
\text { HomoPOA } & (\operatorname{Var}(i, j)<\text { Threshold }) \\
\text { HeteroPOA } & (\operatorname{Var}(i, j)>\text { Threshold })
\end{aligned}\right.
$$

The threshold in Eq. (7) is set by using training data. As a result, urban areas are classified as either homogeneous or heterogeneous.

The influence of POA can be removed by normalizing scattering-power components in each POA space. First, the whole POA space is divided into specific intervals. Then, in each POA interval the average and the standard deviation of each power component's scattering intensity in urban areas are calculated separately for homogeneous and heterogeneous POA areas. Finally, the power component's scattering intensity is normalized for all pixels in each POA interval. According to the results reported by Kajimoto and Susaki (2013b), we selected $P_{v+c}$ as an optimal scattering to represent urban density. The normalized scattering intensity is expressed as follows: 


$$
T_{v+c}(i, j, k)=\frac{P_{v+c}(i, j, k, \phi, H)-\mu_{v+c}(\phi, H)}{\sigma_{v+c}(\phi, H)}
$$

$$
\sigma_{v+c}^{2}(\phi, H)=\frac{1}{N(\phi, H)} \sum_{i} \sum_{j} \sum_{k}\left(P_{v+c}(i, j, k, \phi, H)-\mu_{v+c}(\phi, H)\right)^{2} .
$$

Here, $T$ is the normalized scattering intensity, $P$ is the original scattering intensity, $\mu$ and $\sigma$ are, respectively, the average

In addition, the average and standard deviation may reflect the statistics of the area of interest, but may not be common

$$
T_{v+c}^{\prime}(i, j, k)=\left\{\begin{array}{cl}
0 & \left(T_{v+c}(i, j, k)<-A\right) \\
\frac{T_{v+c}(i, j, k)+A}{2 A} & \left(-A \leq T_{v+c}(i, j, k) \leq A\right) . \\
1 & \left(T_{v+c}(i, j, k)>A\right)
\end{array}\right.
$$

Here, $A$ is a constant. 
and Near Infrared Radiometer type 2 (AVNIR-2) optical sensor data were used as a reference. It is known that L-band SAR observation has significant effects from Faraday rotation, a phenomenon by which the plane of polarization is rotated, especially in tropical regions. This experiment assumed negligible effects of Faraday rotation, because correction was successful. We have two categories of PALSAR data: data with a $21.5^{\circ}$ off-nadir angle against the center of the scene (Data A) and data with a $23.1^{\circ}$ off-nadir angle (Data B). Table 1 shows a listing of Data A and B, respectively.

Accurate information on urban density for Japanese cities was obtained from Zmap-TOWN II (ZENRIN) data, which are residential maps of Japan. Accurate urban density data were generated from Zmap-TOWN II (GIS) data, with reference to previous research (Tanaka, 2011). Two measures of urban density were defined: building-to-land ratio and floor-area ratio. First, building polygon data are intersected by a mesh. Buildings lying across the mesh border are divided into pieces by the border line. The mesh size was tentatively set to $20 \mathrm{~m} \times 20 \mathrm{~m}$, which approximately corresponds to the ground resolution of PALSAR after a multilooking process. Building density is calculated as follows:

$$
\begin{gathered}
D_{\text {Building-to-Land }}(i, j, k)=\frac{\sum_{l=1}^{n} S_{l}(i, j, k)}{S_{\text {Land }}(i, j, k)} \\
D_{\text {Floor }}(i, j, k)=\frac{\sum_{l=1}^{n} S_{l}(i, j, k) \times F_{l}(i, j, k)}{S_{\text {Land }}(i, j, k)} \quad(\boldsymbol{l} \in(i, j, k) \text { pixel }) .
\end{gathered}
$$

Here, $D$ is the estimated building density, $S$ is an area, and $F$ is a building floor. The pair $(i, j)$ is the location of the reference pixel, $k$ indicates the SAR image number, and $l$ denotes the $l$ th building included in the $(i, j)$ pixel. Finally, GIS images were co-registered to PALSAR images by manually selecting ground control points between the images. Coefficients recorded in the leader files of PALSAR data calculate latitude and longitude for each pixel. With these latitudes and longitudes, urban density maps were automatically converted to the WGS 1984 coordinate system with UTM (Universal Transverse Mercator) projection. In this research, the UTM image grid size was set to $25 \mathrm{~m}$.

For cities outside Japan, we used Open Street Map (2014). Shape files of building distributions were available for Munich and New York. We therefore generated building-to-land images for those two cities, and used them for assessment of the estimated urban densities. 


\section{Experiments}

In this study, Lee's sigma filter is applied to PALSAR images as a speckle filter (Lee et al., 2009). The local window size for the filtering was set to $5 \times 5$. In the process of urban area extraction, a $3 \times 3$ boxcar filter was applied to the coherency matrix. The boxcar filter is effective in removing speckle noise but blurs an image quite substantially. However, in urban density estimation, preserving a target signature is a top priority, so Lee's sigma filter with the smallest window size, $5 \times 5$, was selected.

In urban area extraction, we followed the thresholds used in Kajimoto and Susaki (2013a). The minimum change in the urban gravity point was set to $0.01 \mathrm{~dB}$ to terminate the optimization loop. For POA randomness calculations, the window size was set at $31 \times 31$ pixels, and the ratio between the pixel count and the total number of pixels in the window for discriminating between urban and mountainous areas was set to 0.35 . The threshold in Eq. (7) is $185.5^{\circ}$ square, following Kajimoto and Susaki (2013b). The procedure for obtaining this value was determined by considering orthogonal building areas in Tokyo and Sapporo images. After manually determining regions of interest (ROIs) of homogeneous orthogonal building areas in the Tokyo and Sapporo areas, the thresholds, which include 95\% of all pixels belonging to the ROIs, were $188^{\circ}$ square (Tokyo) and $183^{\circ}$ square (Sapporo). The average of the two study area thresholds, $185.5^{\circ}$, was used. Because application of this threshold value to the study areas was successful in the experiments, we did not change the value.

In urban density estimation, A in Eq. (11) was set to 3 to normalize $T$ in Eq. (8).

\subsection{Examination of Two Dataset Differences}

We first examined the differences between the two datasets: Data A and B. Figures 2(a) and (b) show the relation between POA and TP of homogeneous and heterogeneous districts, respectively, in eight Japanese cities. Data A includes the Tokyo metropolitan area (hereafter, "Tokyo"), Kyoto, Nagoya, Sendai, and Kobe, and Data B includes Osaka, Sapporo, and Fukuoka. Figure 2 indicates that there is a significant gap between the curves of Data A and those of Data B. To examine differences between the two datasets in specific areas, we used Data A and B of Tokyo and Sapporo (Table 1). Figures 3(a) and (b) show the relation between POA and TP of homogeneous and heterogeneous districts in the two cities. Figure 3 indicates that the relation is dependent not on the orbital difference (ascending or descending), but on off-nadir angle. Data B (23.1 ${ }^{\circ}$ off-nadir angle) are not reliable because the relations between peaks of the curves of Sapporo A and Tokyo D are inconsistent between homogeneous and heterogeneous districts (Figs. 3(a) 
297

and 3(b)); the peak of the curve of Sapporo A is higher than that of Tokyo D in Fig. 3(a), but this is not the case in Fig. 3(b). This may be due to the quality of the calibration. As a result, we decided to use only Data A for further analysis.

\subsection{Effect of Spatial Scale}

In this research, we used PALSAR images whose ground range resolution is approximately $25 \mathrm{~m}$, and generated urban density maps by aggregating the results of each pixel. As expected, the accuracy obtained at smaller spatial scales (e.g. $1 \mathrm{~km}, 10 \mathrm{~km}$ ) is better than that obtained at larger scales (e.g. $10 \mathrm{~m}, 100 \mathrm{~m}$ ), but the results lose more information. We investigated the optimal spatial scale for maps in terms of accuracy and detail. For accuracy, we examined the correlation coefficients using GIS data at different spatial scales such as 100 m, 200 m, and 300 m, as shown in Figure 4(a). For the map detail, we examined mutual information (Kullback-Leibler information or distance), expressed as

$$
D(P \| Q)=\sum_{i} P(i) \log \frac{P(i)}{Q(i)}
$$

Here, $D(\mathrm{P} \| \mathrm{Q})$ denotes Kullback-Leibler information, and $P$ and $Q$ are discrete probability distributions. In this experiment, we compared the distribution of urban density [0:1] at each spatial scale to that of a $50 \mathrm{~m}$ scale (Figure 4(b)). The interval of urban density for calculating Eq. (1) was set to 0.01. This index represents how much detail is lost with a spatial scale change.

The greatest difficulty in assessing optimal spatial scale is how to combine the correlation coefficient and the mutual information, because the mutual information represents only the relative distance between two probabilistic distributions. Because it seems quite difficult to find a reasonable solution, we did not combine them, but we qualitatively assessed the optimal spatial resolution by referring to the two results. The spatial scale of a thematic map depends on the map purpose. In this research, we decided that the correlation coefficient should not be less than a certain threshold, which we set as 0.7 for all cities. Then, according to Figure 4(a), the optimal spatial scale was selected as 300 m. 


\subsection{Effect of Incident Angle of Radar}

Figure 5 and Table 2 show the effect of incident angle difference on the accuracy of urban density estimation. Note that the results were obtained using slant-range coordinate (original coordinate) images, for ease in calculating the incident angle of each pixel. Because the off-nadir angle was $21.5^{\circ}$ against the scene center, the incident angle at the scene center was approximately $24.0^{\circ}$. The incident angle, $\theta$, was classified into three ranges: $\theta \leq 23.0^{\circ}, 23.5^{\circ} \leq \theta \leq$ $24.5^{\circ}$, and $\theta \geq 25.0^{\circ}$. Table 2 shows the results of correlation coefficient calculations for 300 -m-resolution GIS images. It shows that there is a significant difference among the correlation coefficients of the three ranges.

One approach to correcting the incident angle effect is to divide the backscatter coefficient by $\cos \theta$ (Shimada et al., 2007). We corrected the original fully polarimetric data by multiplying them by the factor $(\cos 24.0 \% \cos \theta)$ and assessed the estimated urban densities with GIS images. As a result, the correlation coefficients became a little worse (by about 0.01) than those without incident angle correction. Although a significant effect of the incident angle difference was found, it may not be simple to remove it. This improvement is left as a future task for mapping urban densities from SAR images.

\subsection{Accuracy Assessment of Urban Density Estimation}

Figures 6, 7, 8, and 9 show the respective results for Tokyo, Kyoto, Munich, and New York, two Japanese cities and two non-Japanese cities. We selected these Japanese cities because Tokyo is a highly dense city; Kyoto is relatively homogeneous in terms of building height due to building regulations. In Figures 6 and 7, panel (a) shows the AVNIR-2 image, (b) and (e) show the estimated urban density from PALSAR images, (c) and (f) show the building-to-land ratio, and (d) and (g) show the floor-area ratio. In Figures 8 and 9, panel (a) shows the AVNIR-2 image, (b) and (d) show the estimated urban density from PALSAR images, and (c) and (e) show the building-to-land ratio. Floor-area ratio data were not available for Munich or New York.

The effect of the mean and standard deviation (Eqs. (9) and (10)) on the final results was examined. In this study, we defined a calibration that calculates the mean and standard deviation over all images used for the analysis, following Eqs. (9) and (10). Figures 10(a) and 11(a) show scattergrams of GIS data and the results before calibration, meaning that the mean and standard deviation used for Eq. (8) were calculated within the individual scene. In contrast, Figs. 10(b) and 11(b) show the scattergram of GIS data and the results after calibration. These results were obtained with a $300 \mathrm{~m}$ 
resolution. Table 3 shows the results of correlation coefficient calculations for $300 \mathrm{~m}$ resolution with using GIS images.

On the whole, correlations with the building-to-land ratio are higher than those with the floor-area ratio. This means that building-to-land ratio is better than floor-area ratio for estimating urban density from PolSAR images..

This paper extends the method previously proposed in Kajimoto and Susaki (2013b) to application for multi-scenes.

The technique calculates statistics for multiple images, and then applies them to all multi-scene images. While this improvement might seem small, it has two important aspects from a statistical viewpoint. The first is normalization of the data. The previously proposed method is based on correction of POA effects in backscattering and related components. For instance, when the POA interval is set to $1^{\circ}$, we take samples and calculate statistics (mean and standard deviation) for $-45^{\circ},-44^{\circ}, \ldots, 44^{\circ}, 45^{\circ}$ POA. The sum of volumetric and helix scatterings is normalized by Eq. (8). Assume that we separately generate two urban density maps for two images. Because the statistics of the two images are different, the values have different meanings. Thus, urban density maps generated in this way are not comparable. Calculating statistics based on multiple images, however, enables generation of comparable urban density maps.

POA. Of course, some POAs have a small number or no samples for a given scene. POA statistics derived from a small number of samples are unstable. This instability has less effect when the method is applied to one scene for estimating to correct POA effects in another, however, the instability becomes significant. We thus need samples from all POAs to generate sufficiently stable statistics for POA effect correction. Even given a certain number of samples for a specific POA from one scene, the proposed method takes samples from multi-scenes to calculate statistics. The statistics from multi-scenes may not be optimal for any individual scene, but they are of use in reducing error caused by applying the statistics from one scene to another.

We examined the effect of the number of cities used to calculate statistics. The results became stable when the number of cities was around 10, and adding additional cities resulted in little improvement. This indicates that robust estimation of urban density in multiple images requires a certain number of images, but that robustness can be achieved when sufficient samples for each POA are obtained.

Here again, we discuss the implications of Table 3. The calibration of mean and standard deviation contributed to a slight improvement of correlation with the building-to-land ratio (by 0.026 for 7 cities), and with the floor-area ratio (by 
0.025 for 5 cities). Figures 10 and 11 show that changes in the estimated urban density caused by calibration are significant for some data in Tokyo and Sendai. The correlation coefficients of some areas after calibration were worse than those before calibration. The average and standard deviation (Eqs. (9) and (10)) were calculated for each area before calibration, and thus they may be optimal for the area. However, an important point is that generated urban density maps are not comparable because of the normalization by using the statistics specific to the area. After calibration, the statistics common to all 17 areas were used to normalize. Because this normalization functioned to shift plots of each city to a common line (Figs. 10(a) to 10(b) and Figs. 11(a) to 11(b)), it contributed to overall improvement of the correlation. Because our objective is to compare the urban densities of global megacities, such normalization is necessary. In this context, in Table 3, the results of overall scenes are much more important than those of each scene. While the improvement indicated by correlation coefficients was small, we continued to apply the proposed method to global megacities.

\subsection{Estimation of Urban Density of Global Megacities}

The urban densities of megacities in ascending-mode Data A (Table 1) are shown in Fig. 12. The images correspond to the areas of $20 \mathrm{~km} \times 20 \mathrm{~km}$. In addition to the images, we extended the proposed method to extracting meaningful statistics in areas and districts. Two sizes of the urban area were set to $10 \mathrm{~km} \times 10 \mathrm{~km}$ and $20 \mathrm{~km} \times 20 \mathrm{~km}$. As for the districts, two sizes were set to $2.5 \mathrm{~km} \times 2.5 \mathrm{~km}$ and $5 \mathrm{~km} \times 5 \mathrm{~km}$, but the center of the window was common to all window sizes. These sizes were selected as follows. We first set a district of $2.5 \mathrm{~km} \times 2.5 \mathrm{~km}$, and this district was automatically determined by examining the highest mean urban densities within the window. This size was determined by examining the size of the highest urban density district in multiple images. The $20 \mathrm{~km}$ size for areas was determined by considering the area covered by PALSAR images. In polarimetric measurement mode, a PALSAR swath is 20 to 65 $\mathrm{km}$ (JAXA, 2006), and the images used in the experiment have approximately $30 \mathrm{~km}$ swaths. It is ideal that the whole of a city should be extracted and compared with those of other cities. However, it was found that parts of some cities were not observed in the PALSAR images. Therefore, we decided to limit the area to compare global megacities. Two different sizes for districts and areas were set because the scattergram depended on the size and the comparison between the results with two different sizes may indicate information about urban distribution patterns. The other area size, 10 $\mathrm{km} \times 10 \mathrm{~km}$, was determined by halving each dimension of $20 \mathrm{~km} \times 20 \mathrm{~km}$. In the same manner, if we halve the $2.5 \mathrm{~km}$ 
403

404

405

406

407

408

409

410

411

412

413

414

415

416

417

418

419

420

421

422

423

424

425

426

427

428

$\times 2.5 \mathrm{~km}$ size of the district we get $1.25 \mathrm{~km} \times 1.25 \mathrm{~km}$, which is too small to represent urban density. We use therefore use $5 \mathrm{~km} \times 5 \mathrm{~km}$, obtained by doubling each dimension of the $2.5 \mathrm{~km} \times 2.5 \mathrm{~km}$ area size.

In calculating the mean, aggregated urban densities were divided by the number of samples where those urban densities were more than 0 . Then, it was visually checked whether the highest urban density district is included in the automatically selected district. All results except the one for Kobe were acceptable. In the case of the Kobe image, parts of the Osaka area were included, and the automatically selected district belonged to them. The highest urban density district for Kobe was automatically detected by limiting the search area. Finally, the area that includes the pre-determined district and shows the highest mean urban densities was detected for each scene. Figure 13 shows the relation between mean urban density in a district (for each, either $2.5 \mathrm{~km} \times 2.5 \mathrm{~km}$ or $5 \mathrm{~km} \times 5 \mathrm{~km}$ ) and the skew of urban density in a wider area $(10 \mathrm{~km} \times 10 \mathrm{~km}$ or $20 \mathrm{~km} \times 20 \mathrm{~km})$. Skew is a statistical measure of asymmetry of a distribution, defined as follows:

$$
\text { Skew }=E\left[\left(\frac{X-\mu}{\sigma}\right)^{3}\right]
$$

Here, $X$ is a random variable, and $\mu$ and $\sigma$ are the mean and standard deviation of $X$, respectively. When the distribution has strong symmetry, the absolute value of the skew is close to 0 .

Figure 13 indicates several interesting findings. The vertical axis of Fig. 13 denotes homogeneity of urbanization, with larger values indicating more heterogeneously urbanized and developed cities in specific districts. The first finding is that cities such as Melbourne and Sydney show local heterogeneity because their skews are relatively high at both spatial scales $(10 \mathrm{~km} \times 10 \mathrm{~km}$ and $20 \mathrm{~km} \times 20 \mathrm{~km})$. This finding is supported by Figs. 13(d) and 13(j). The second finding is that the skew change indicates the degree of homogeneity. The skew of Ho Chi Minh City significantly increased between the $10 \mathrm{~km}$ and $20 \mathrm{~km}$ scales. This feature is unique to Ho Chi Minh City. It indicates that homogeneous areas with higher urban densities are distributed on a $10 \mathrm{~km}$ scale and that urban densities are significantly different between inside and outside the highly urbanized $10 \mathrm{~km} \times 10 \mathrm{~km}$ area. Such a homogeneous area can be found in the left area of Fig. 13(b). The white triangle shows the international airport in Ho Chi Minh City. On the other hand, Tokyo, Taipei, Tehran, and Kyoto have small skew change between the $10 \mathrm{~km}$ and $20 \mathrm{~km}$ scales. This means that the homogeneous urban areas are found in a $20 \mathrm{~km} \times 20 \mathrm{~km}$ area in these cities. 
The third finding is that a few cities can be classified into same categories having similar urban structure by considering both plots in two scattergrams: (1) Tokyo and Taipei, (2) Munich and Beijing, (3) Kyoto and Tehran, (4) Melbourne and Sydney, and (5) Sendai and New Delhi. These similarities are also seen in Figs. 6, 7, 8 and 12. The final finding is that Vientiane, the capital and the largest city in the Laos, is much less urbanized than other cities in terms of urban density on a district level and an area level. On the basis of the previous discussion, we can compare the status of different global megacities by using PolSAR images and the proposed method.

\section{Conclusions}

We extended an existing effective density estimation algorithm to allow application to various areas, while the existing one was limited to application to single areas. A normalized combination of the volume scattering power and the helix scattering power $(T v+c)$ was used to calculate urban density. The mean and standard deviation used for the normalization were obtained by a calibration referring to all images to be analyzed. As a result of validation with GIS images, a small improvement was confirmed and the urban density estimated from a single PolSAR image has a significant correlation with the building-to-land ratio. We then applied this improved method to global megacities, and generated a two-dimensional scattergram of mean and skew of urban densities. This scattergram enabled international comparison of megacities in terms of urban structure, and indicated several findings. As a result, we found that the proposed method and such discussion based on the scattergram were very useful in obtaining knowledge about the status of megacities, especially when fundamental statistics are lacking for megacities of interest.

In this study, we used L-band PALSAR images because fully polarimetric PALSAR images were available for many megacities over the world, and because stable results of urban mapping using L-band PolSAR images have been reported (Kajimoto \& Susaki, 2013b). Satellite-borne X-band PolSAR images, such as those taken by TerraSAR-X, are now available, and the proposed method may be applied to such images. However, it may be expected that the obtained results will be different from those obtained as L-band images because the radar sensitivity of scatterers is dependent on wavelength. Because multiple scattering frequently occurs in urban areas, longer wavelength radar may be more appropriate for urban densities that have high correlation with building-to-land ratio. In future work, we will compare urban density maps generated from L-band PolSAR images with those generated from X-band PolSAR images. 
456

457

458

459

460

461

462

463

464

465

466

467

468

469

Acknowledgments

This research was supported by a Grant-in-Aid for Scientific Research (KAKENHI) for Young Scientists (B) (No. 22760393), and by a program of the Fourth Advanced Land Observing Satellite-2 Research Announcement, Japanese Aerospace Exploration Agency. Zmap-Town II (ZENRIN) was provided by the Center for Spatial Information Science, The University of Tokyo.

\section{References}

Bagan, H., \& Yamagata, Y. (2012) Landsat analysis of urban growth: How Tokyo became the world's largest megacity during the last 40 years. Remote Sensing of Environment, 127, 210-222.

Chaussard, E., Wdowinski, S., Cabral-Cano, E., \& Amelung, F. (2014) Land subsidence in central Mexico detected by ALOS InSAR time-series, Remote Sensing of Environment, 140, 94-106.

Elvidge, C. D., Baugh, K. E., Kihn, E. A., Kroehl, H. W., \& Davis, E. R. (1997) Mapping City Lights with nighttime data from the DMSP operational linescan system. Photogrammetric Engineering \& Remote Sensing, 63, 727-734.

Elvidge, C. D., Baugh, K. E., Dietz, J. B., Bland, T., Sutton, P. C., \& Kroehl, H. W. (1998) Radiance calibration of DMSP-OLS low-light imaging data of human settlements. Remote Sensing of Environment, 68, 77-88.

Ferretti, A., Prati, C., \& Rocca, F. (2000). Non-linear subsidence rate estimation using permanent scatterers in differential SAR Interferometry. IEEE Transactions on Geoscience and Remote Sensing, 38, 2202-2212.

Ferretti, A., Prati, C. \& Rocca, F. (2001) Permanent scatterers in SAR interferometry. IEEE Transactions on Geoscience and Remote Sensing, 39, 8-20.

Ferretti, A., Fumagalli, A., Novali, F., Prati, C., Rocca, F. \&Rucci, A. (2011) A new algorithm for processing interferometric data-stacks: SqueeSAR. IEEE Transactions on Geoscience and Remote Sensing, 49, 3460-3470. 
Freeman, A. \& Durden, S. L. (1998) A three-component scattering model for polarimetric SAR data. IEEE Transactions on Geoscience and Remote Sensing, 36, 936-973.

Friedl, M. A., McIver, D. K., Hodges, J. C. F., Zhang, Z. Y., Muchoney, D., Strahler, A. H., Woodcock, C. E., Gopal, S., and early results. Remote Sensing of Environment, 83, 287-302.

490

Iwasa, S. \& Susaki, J. (2011) Classification of building area using azimuth angle and density indices derived from polarimetric SAR. Proceedings of Joint Urban Remote Sensing Event, 269-272.

JAXA (2006), About ALOS - PALSAR. Available at http://www.eorc.jaxa.jp/ALOS/en/about/palsar.htm last accessed: Jul 14, 2014.

496

Kajimoto, M. and Susaki, J. (2013a) Urban area extraction from polarimetric SAR images using polarization orientation angle. IEEE Geoscience Remote Sensing Letters, 10, 337-341.

Kajimoto, M., \& Susaki, J. (2013b) Urban density estimation from polarimetric SAR images based on a POA correction method. IEEE Journal of Selected Topics in Applied Earth Observations and Remote Sensing, 6, 1418-1429.

Kimura, H. (2008) Radar polarization orientation shifts in built-up areas. IEEE Geoscience Remote Sensing Letters. 5, 217-221.

505

Lee, J. S., Wen, J. H., Ainsworth, T. L., Chen, K. S., \& Chen, A. J. (2009) Improved sigma filter for speckle filtering of 508

Margarit, G., Mallorquí, J. J., \& Pipia, L. (2010) Polarimetric characterization and temporal stability analysis of urban target scattering, IEEE Transactions on Geoscience and Remote Sensing, 48, 2038-2048. 
512 Niu, X., \& Ban, Y. (2012) An adaptive contextual SEM algorithm for urban land cover mapping using multitemporal 513 high-resolution polarimetric SAR data. IEEE Journal of Selected Topics in Applied Earth Observations and Remote $514 \quad$ Sensing. 5, 1129-1139.

515

OpenStreetMap Data Extracts, Open Street Map (2014) Available at http://download.geofabrik.de/ last accessed: Jul 13,

Perissin, D. \& Wang, T. (2012) Time-series InSAR applications over urban areas in China. IEEE Journal of Selected Topics in Applied Earth Observations and Remote Sensing. 4, 92-100.

Schneider, A., Friedl, M. A., \& Potere, D. (2010) Mapping global urban areas using MODIS 500-m data: New methods and datasets based on 'urban ecoregions.' Remote Sensing of Environment, 114, 1733-1746.

524

Shabou, A., Baselice, F., \& Ferraioli, G. (2012) Urban digital elevation model reconstruction using very high resolution multichannel InSAR data. IEEE Transactions on Geoscience and Remote Sensing, 50, 4748-4758.

Shimada, M., Isoguchi, O., Tadono, T., Higuchi, R. \& Isono, K. (2007) PALSAR CALVAL summary and update 2007. 533

Proceedings of IEEE International Geoscience and Remote Sensing Symposium 2007, 3593-3596. 
Stramondo, S., Bozzano, F., Marra, F., Wegmuller, U., Cinti, F.R., Moro, M. \& Saroli, M. (2008) Subsidence induced

by urbanisation in the city of Rome detected by advanced InSAR technique and geotechnical investigations, Remote

Sensing of Environment, 112, 3160-3172.

540

Sutton, P. C. (2003) A scale-adjusted measure of "Urban sprawl” using nighttime satellite imagery. Remote Sensing of Environment, 86, 353-369.

543

Tanaka, K. (2011) The Land Institute of Japan, 2011. Formulation of urban density indices by using geospatial information: A case of Tokyo Metropolitan Area. Tokyo: The Land Institute of Japan, pp. 1-49, Japanese.

Thiele, A., Cadario, E., Schulz, K., Thönnessen, U., \& Soergel, U. (2007) Building recognition from multi-aspect high-resolution InSAR data in urban areas. IEEE Transactions on Geoscience and Remote Sensing, 45, 3583-3593.

Yamaguchi, Y., Moriyama, T., Ishido, M., \& Yamada, H. (2005) Four-component scattering model for polarimetric

SAR image decomposition. IEEE Transactions on Geoscience and Remote Sensing, 43 1699-1706.

Yamaguchi, Y., Yajima, Y., \& Yamada, H. (2006) A four-component decomposition of POLSAR images based on the coherency matrix. IEEE Geoscience Remote Sensing Letters, 3, 292-296.

Yamaguchi, Y., Sato, A., Boerner, W., Sato, R., \& Yamada, H. (2011) Four-component scattering power decomposition with rotation of coherency matrix. IEEE Transactions on Geoscience and Remote Sensing, 49, 2251-2258.

Zenrin, Co. Ltd. (2014) Zmap Town II. Available at http:// www.zenrin.co.jp/product/gis/zmap/zmaptown.html (in Japanese) lass accessed: Jan 5, 2014.

Zhu, Z., Woodcock, C. E., Rogan, J., \& Kellndorfer, J. (2012) Assessment of spectral, polarimetric, temporal, and 
566

567

568

569

570

571

572

573

574

575

576

577

578

579

580

581

582

583

584

585

586

587

588

589

590

591

\section{List of Figure Captions}

Fig. 1. Flow of the proposed method

Fig. 2. Average TP of PALSAR images plotted against POA. (a) Average TP for homogeneous POA areas, and (b) average TP for heterogeneous POA areas. Data A with $21.5^{\circ}$ off-nadir angle against scene center includes Tokyo, Kyoto, Sendai, Nagoya, and Kobe. Data B with $23.1^{\circ}$ off-nadir angle includes Osaka, Fukuoka, and Sapporo.

Fig. 3. Average TP of PALSAR images (Sapporo and Tokyo) plotted against POA. Each city has two images with different off-nadir angle. (a) Average TP for homogeneous POA areas, and (b) average TP for heterogeneous POA areas. "A" denotes ascending mode of observation, and "D" denotes descending mode of observation.

Fig. 4. Effect of spatial scale on the results. (a) Correlation coefficient of estimated urban density between SAR data and GIS data, (b) mutual information (Kullback-Leibler information) compared to the data at a $50 \mathrm{~m}$ spatial scale.

Fig. 5. Effect of incident angle $\theta$ difference to the accuracy of urban density estimation. (a) Results in case when $\theta \leq$ $23.0^{\circ}$, (b) $23.5^{\circ} \leq \theta \leq 24.5^{\circ}$, and (c) $\theta \geq 25.0^{\circ}$.

Fig. 6. Results of urban density estimation for Tokyo. (a) AVNIR-2 image observed on January 11, 2007 (R:G:B = band 3:4:2), (b)(e) estimated urban density, (c)(f) building-to-land ratio, and (d)(g) floor area ratio. (b), (c), and (d) were original data, and aggregated into images with $300 \mathrm{~m}$ mesh size (e), (f), and (g), respectively.

Fig. 7. Results of urban density estimation for Kyoto. See Fig. 4 for a description of each panel. The AVNIR-2 image was observed on May 15, 2008. Note that some urban areas in (a) are not included in (c) and (d), because (c) and (d) have only data from inside Kyoto. 
592

593

594

595

596

597

598

599

600

601

602

603

604

605

606

607

608

609

610

611

612

Fig. 8. Results of urban density estimation for Munich. (a) AVNIR-2 image observed on September 22, 2010 (R:G:B = band 3:4:2), (b)(d) estimated urban density, and (c)(e) building-to-land ratio. (b) and (c) were original data, and aggregated into images with $300 \mathrm{~m}$ mesh size (d) and (e), respectively.

Fig. 9. Results of urban density estimation for New York. See Fig. 7 for a description of each panel. The AVNIR-2 image was observed on November 3, 2010.

Fig. 10. Effect of calibration to the assessment of the estimated urban density with building-to-land ratio. (a) Scattergram of GIS data and the results before calibration, in which the mean and standard deviation used for Eq. (8) were calculated within the individual scene. (b) Scattergram of GIS data and the results after calibration, in which the mean and standard deviation were calculated using Eqs. (9) and (10), respectively.

Fig. 11. Effect of calibration to the assessment of the estimated urban density with floor area ratio. See Fig. 9 for a description of each panel.

Fig. 12. Results of urban density estimation. (a) Beijing, (b) Ho Chi Minh, (c) Kobe, (d) Melbourne, (e) Nagoya, (f) New Delhi, (g) Sendai, (h) Shanghai, (i) Singapore, (j) Sydney, (k) Taipei, (l) Tehran, and (m) Vientiane.

Fig. 13. Scattergram of indices derived from estimated urban densities. (a) Relation between the highest mean in a 2.5 $\times 2.5 \mathrm{~km}$ district and the skew of urban density in a $10 \times 10 \mathrm{~km}$ area, and (b) relation between the highest mean in a 5 $\times 5 \mathrm{~km}$ district and the skew of urban density in a $20 \times 20 \mathrm{~km}$ area. 
Table 1: Details of POLSAR images used for the experiment. All images except those with notation "descending" were observed in an ascending mode.

\begin{tabular}{|c|c|c|c|}
\hline \multicolumn{4}{|c|}{ Data A (21.5 off-nadir angle against scene center) } \\
\hline City & $\begin{array}{l}\text { Observation date } \\
\text { (yyyy/mm/dd) }\end{array}$ & City & $\begin{array}{c}\text { Observation date } \\
\text { (yyyy/mm/dd) }\end{array}$ \\
\hline Tokyo & 2006/07/17 & New Delhi & $2010 / 03 / 28$ \\
\hline Kyoto & $2007 / 06 / 02$ & New York & $2011 / 04 / 01$ \\
\hline Nagoya & $2010 / 11 / 07$ & Shanghai & $2011 / 03 / 29$ \\
\hline Kobe & $2007 / 05 / 04$ & Singapore & $2007 / 06 / 01$ \\
\hline Sendai & $2009 / 04 / 19$ & Sydney & $2007 / 05 / 04$ \\
\hline Beijing & $2011 / 04 / 08$ & Taipei & $2011 / 04 / 03$ \\
\hline Ho Chi Minh & $2007 / 04 / 01$ & Tehran & $2009 / 04 / 23$ \\
\hline Melbourne & $2011 / 04 / 07$ & Vientiane & $2007 / 05 / 10$ \\
\hline Munich & $2011 / 03 / 20$ & Sapporo (descending) & $2008 / 07 / 26$ \\
\hline \multicolumn{4}{|c|}{ Data B $\left(23.1^{\circ}\right.$ off-nadir angle against scene center $)$} \\
\hline City & $\begin{array}{l}\text { Observation date } \\
\text { (yyyy/mm/dd) }\end{array}$ & City & $\begin{array}{c}\text { Observation date } \\
\text { (yyyy/mm/dd) }\end{array}$ \\
\hline Osaka & 2009/05/09 & Kalach & $2010 / 05 / 02$ \\
\hline Fukuoka & $2009 / 06 / 10$ & Kolkata & $2010 / 05 / 29$ \\
\hline Sapporo & $2007 / 05 / 25$ & Paris & $2009 / 05 / 12$ \\
\hline Bangkok & $2010 / 05 / 28$ & Yangon & $2010 / 05 / 09$ \\
\hline Hanoi & $2010 / 06 / 06$ & Tokyo (descending) & $2006 / 08 / 19$ \\
\hline Jakarta & $2010 / 05 / 06$ & & \\
\hline
\end{tabular}


Table 2: Effect of incident angle difference to correlation coefficients of estimated urban density between SAR data $\left(T_{v+c}\right)$ and GIS data. Five cities (Tokyo, Kyoto, Nagoya, Kobe, and Sendai) were used for the analysis.

\begin{tabular}{|c|r|r|r|r|r|}
\hline & \multicolumn{2}{|c|}{ Before calibration } & \multicolumn{2}{c|}{ After calibration } & \multirow{2}{*}{$\begin{array}{c}\text { Sample } \\
\text { (pixel) }\end{array}$} \\
\hline & $\begin{array}{c}\text { Building-to-lan } \\
\text { d ratio }\end{array}$ & $\begin{array}{c}\text { Floor area } \\
\text { ratio }\end{array}$ & $\begin{array}{c}\text { Building-to-1 } \\
\text { and ratio }\end{array}$ & $\begin{array}{c}\text { Floor area } \\
\text { ratio }\end{array}$ & 6288 \\
\hline$\theta \leq 23.0 \mathrm{deg}$ & 0.818 & 0.676 & 0.837 & 0.686 & 3576 \\
\hline $23.5 \leq=\theta \leq 24.5$ & 0.778 & 0.617 & 0.797 & 0.660 & 4585 \\
\hline$\theta \geq 25.0$ & 0.757 & 0.580 & 0.764 & 0.586 & \\
\hline
\end{tabular}

Table 3: Correlation coefficients of estimated urban density between SAR data $\left(T_{v+c}\right)$ and GIS data at a $300 \mathrm{~m}$ spatial scale.

\begin{tabular}{|c|r|r|r|r|}
\hline & \multicolumn{2}{|c|}{ Before calibration } & \multicolumn{2}{c|}{ After calibration } \\
\cline { 2 - 5 } & $\begin{array}{c}\text { Building-to-land } \\
\text { ratio }\end{array}$ & Floor area ratio & $\begin{array}{c}\text { Building-to-land } \\
\text { ratio }\end{array}$ & Floor area ratio \\
\hline Tokyo & 0.731 & 0.560 & 0.740 & 0.550 \\
\hline Kyoto & 0.817 & 0.673 & 0.811 & 0.665 \\
\hline Nagoya & 0.621 & 0.468 & 0.620 & 0.461 \\
\hline Kobe & 0.726 & 0.642 & 0.723 & 0.640 \\
\hline Sendai & 0.741 & 0.575 & 0.739 & 0.574 \\
\hline Munich & 0.661 & N/A & 0.657 & N/A \\
\hline New York & 0.607 & N/A & 0.602 & N/A \\
\hline All & 0.702 & 0.553 & 0.728 & 0.578 \\
\hline
\end{tabular}




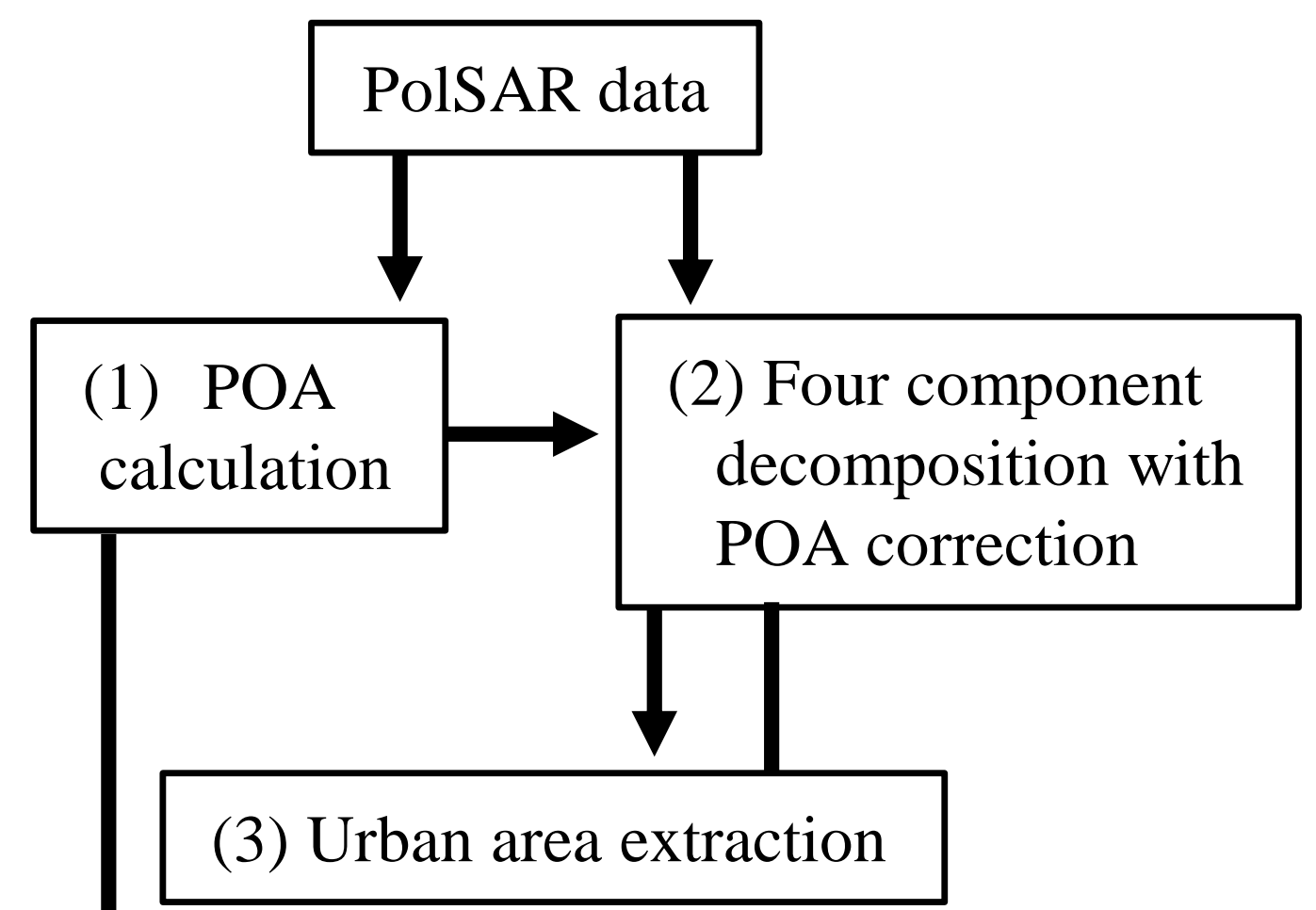

(3-1) Classification using $P_{v}$ and $T P$

(3-2) Classification using POA randomness

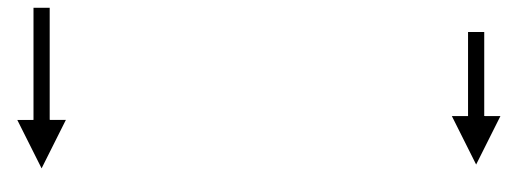

(4) Urban density estimation

(4-1) Classification into homogenous and heterogeneous POA districts

(4-2) Calculation of statistics of $P_{v+c}$ by POA for all study areas

(4-3) Calculation of urban density by normalization of $P_{v+c}$

(4-4) Calculation of indices derived from urban density $\left(D_{1}\right.$ and $\left.D_{2}\right)$ in regions of interest 


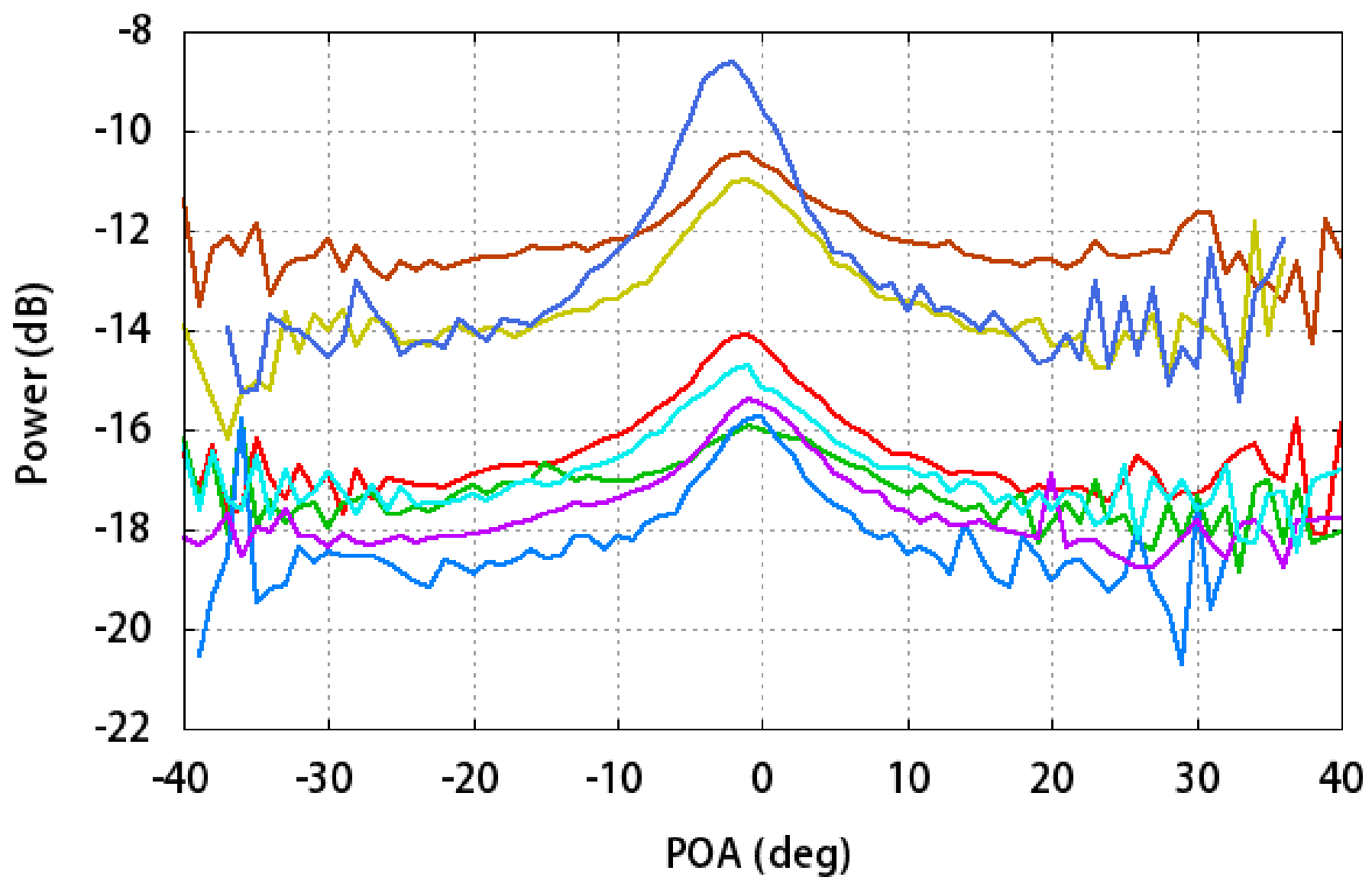

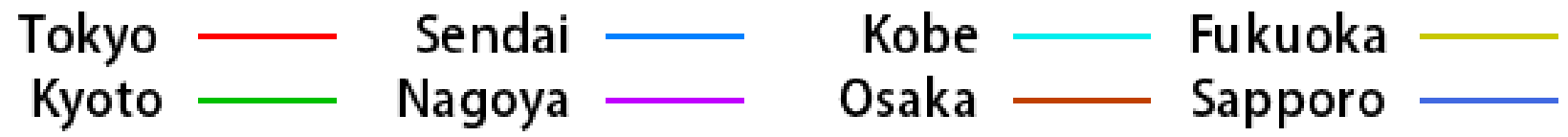




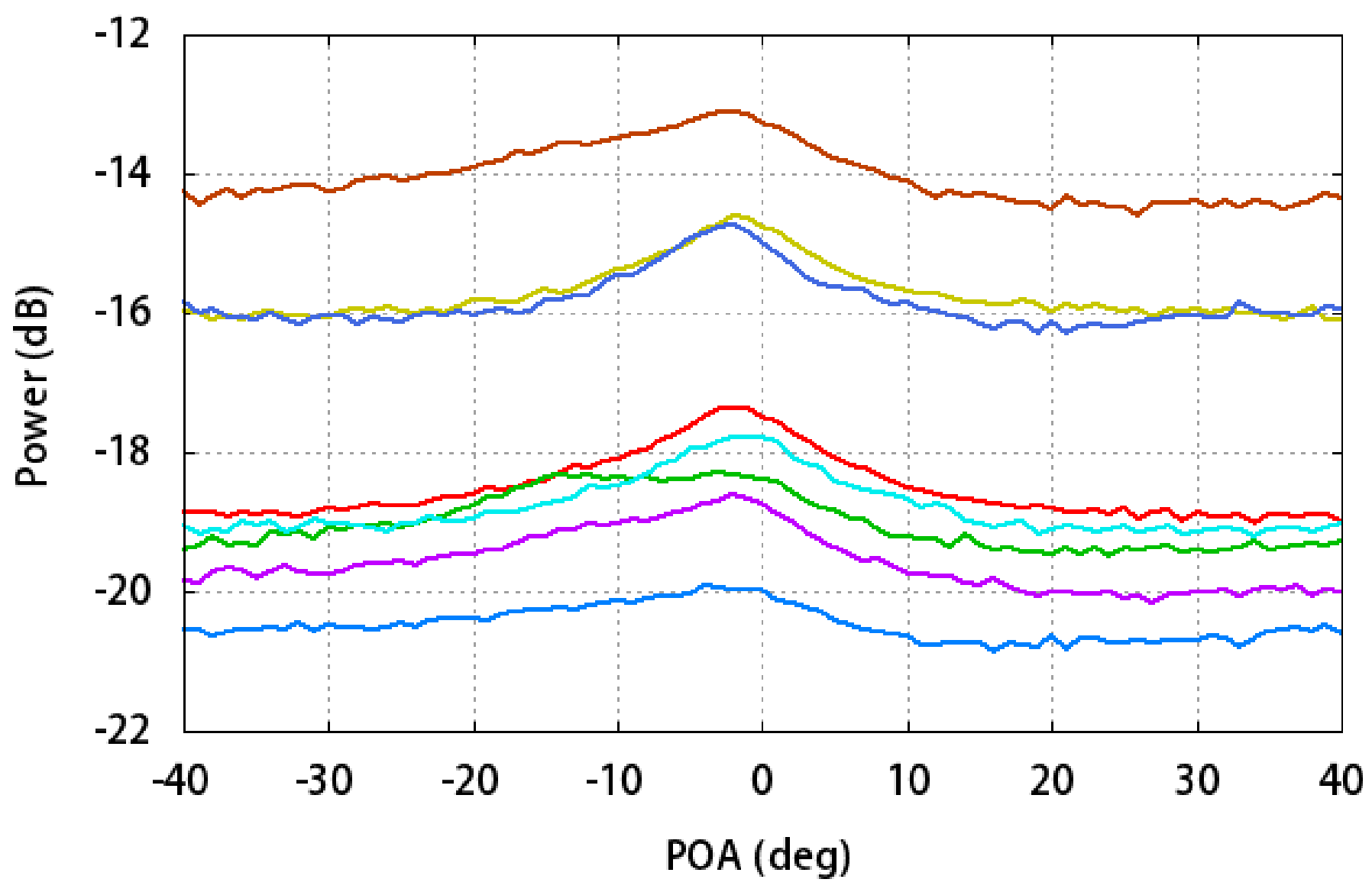

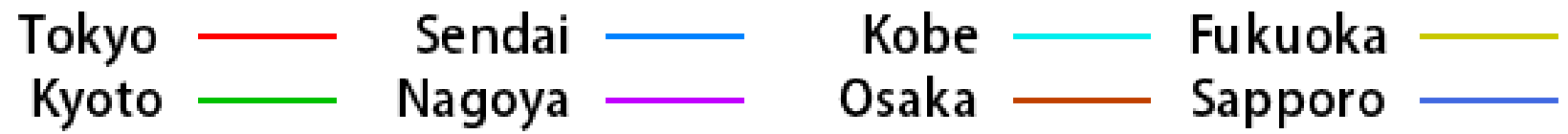

(b) 


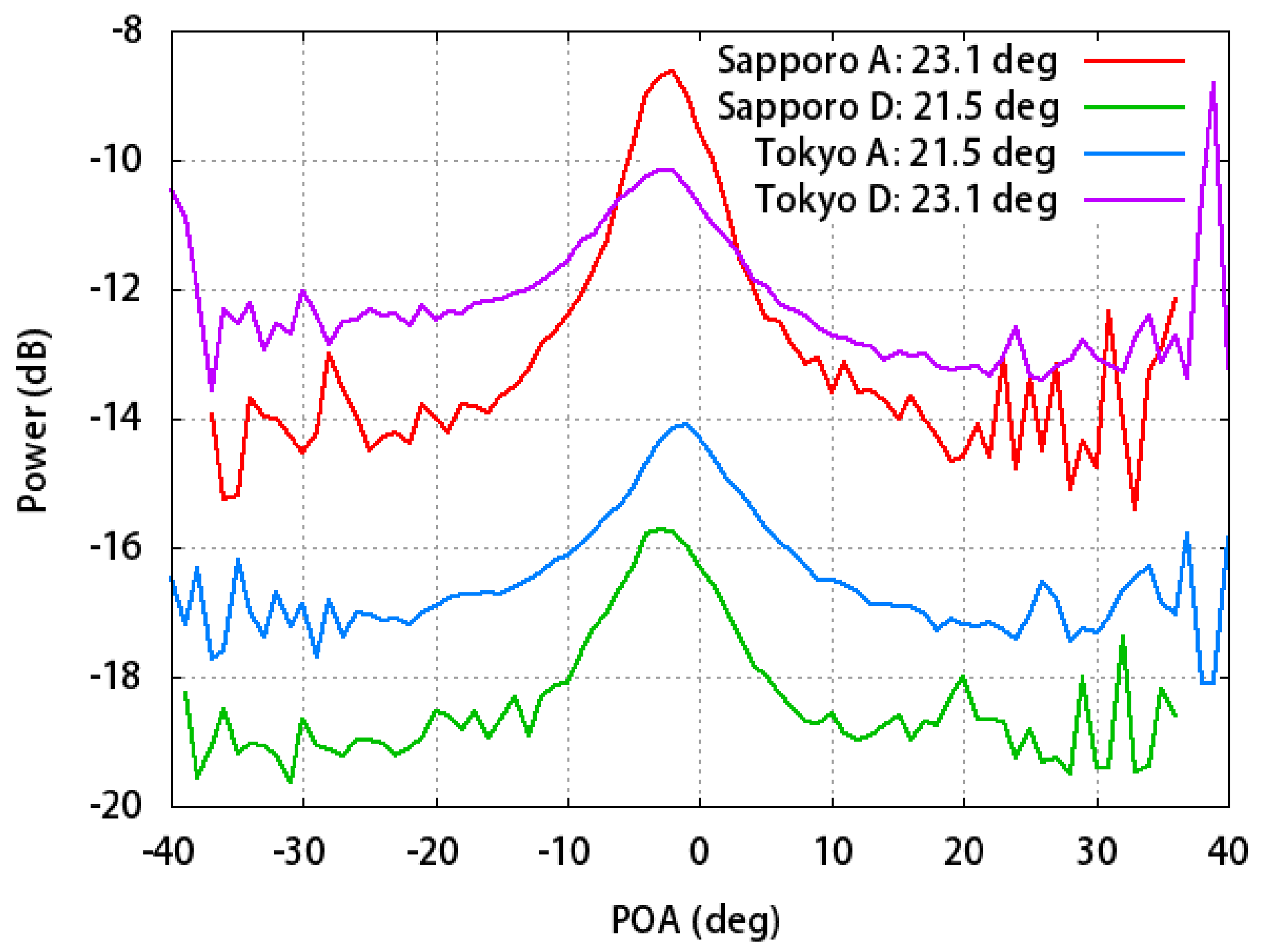

(a) 


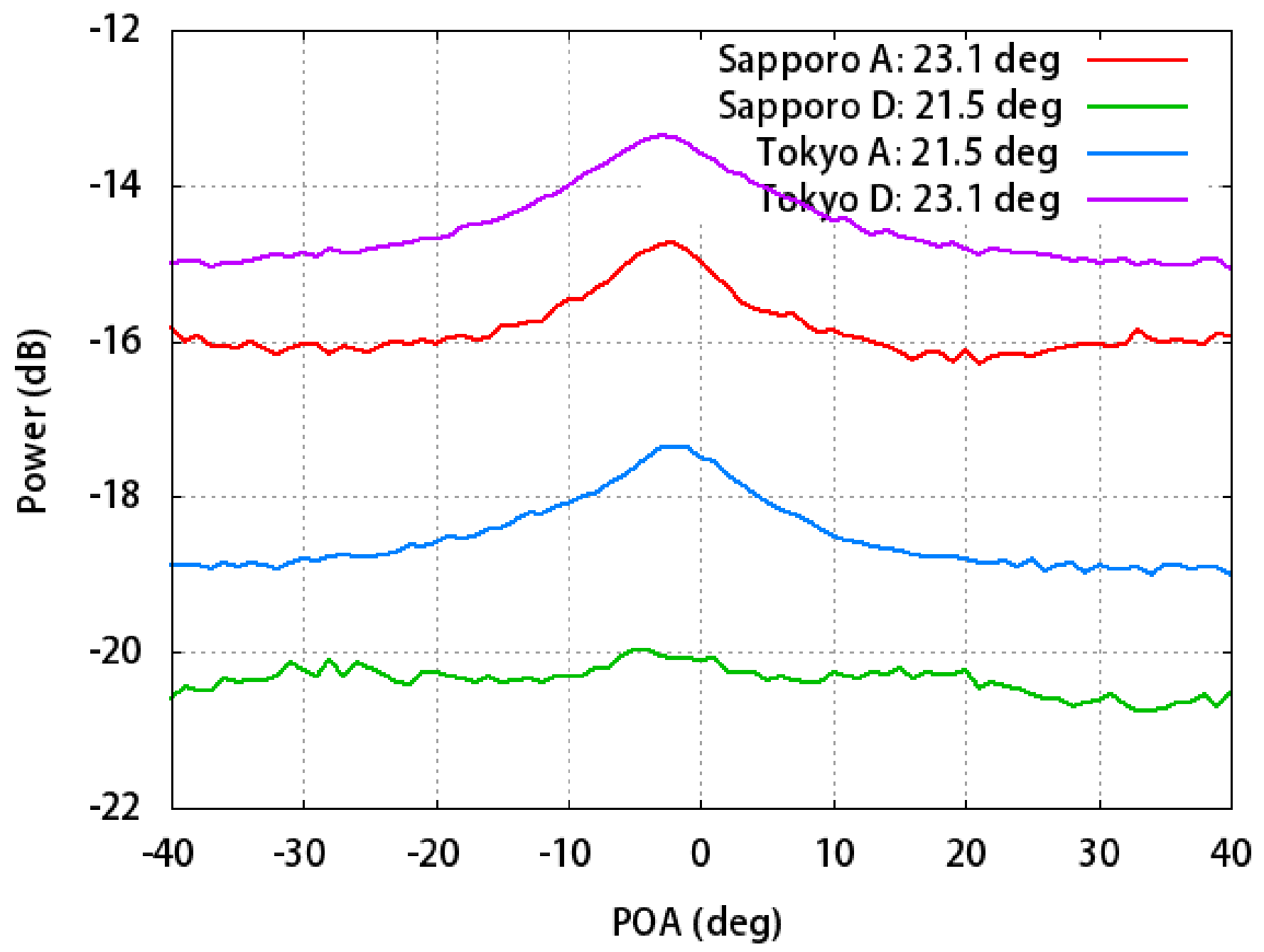

(b) 


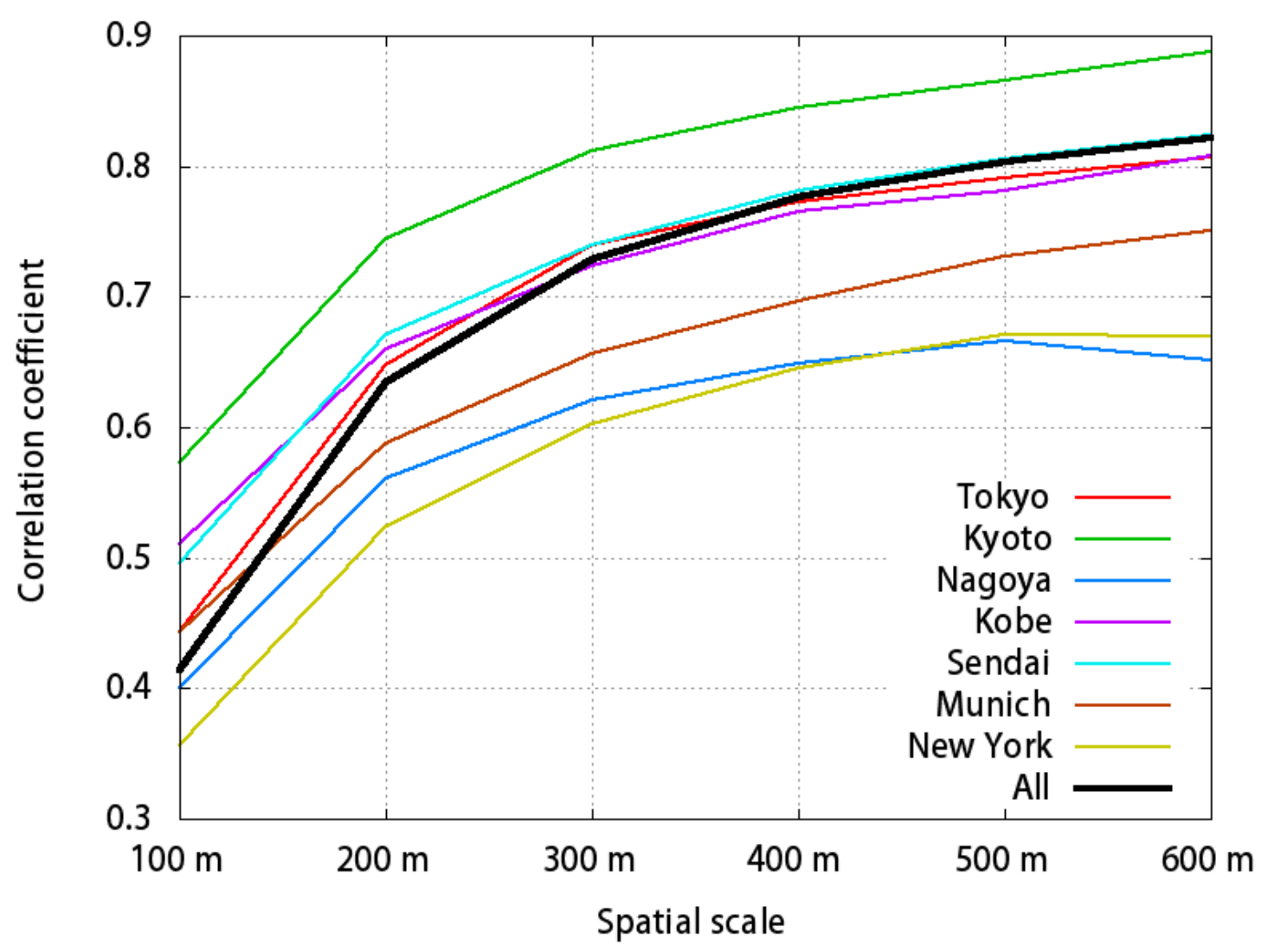

(a) 


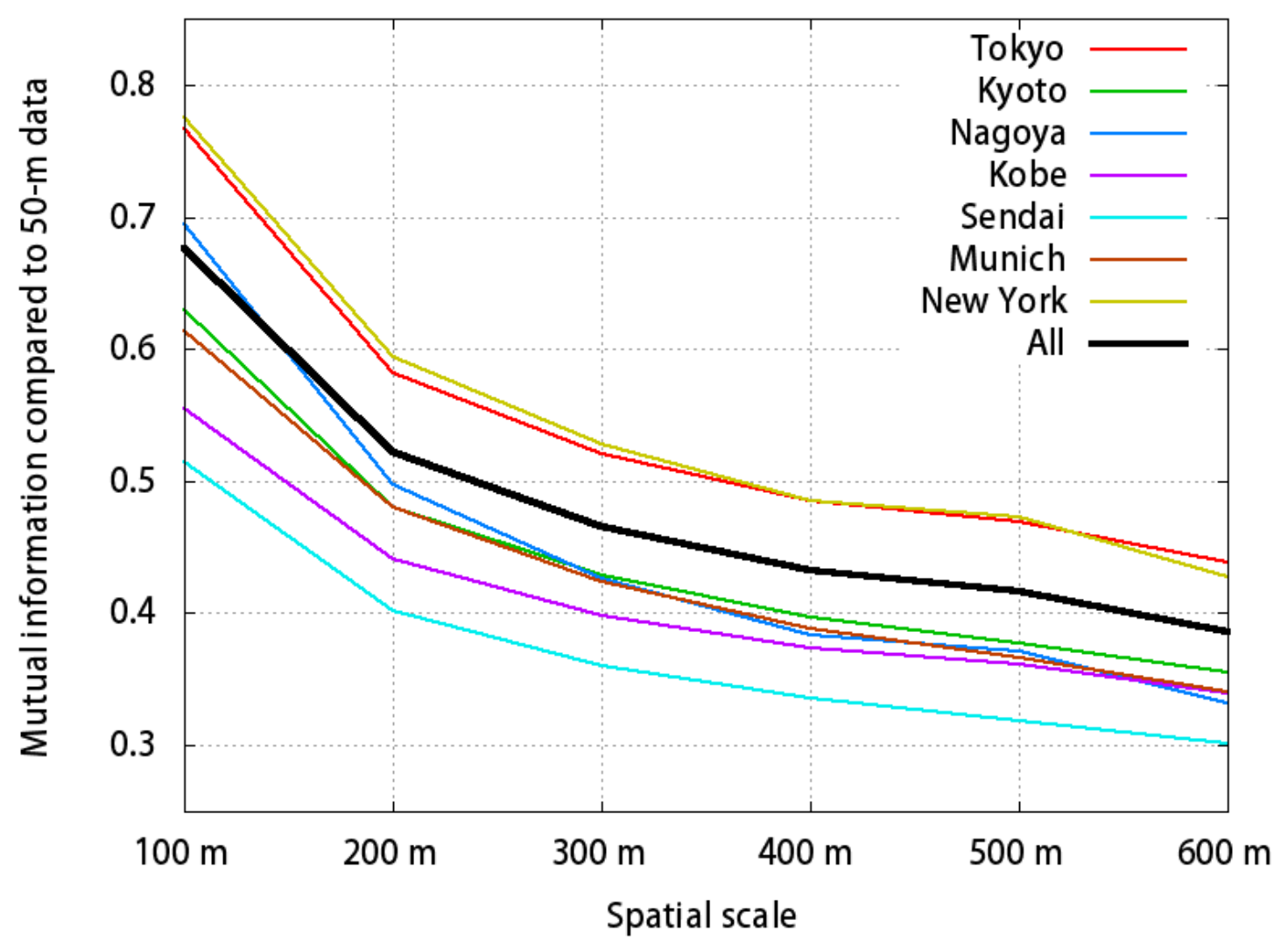

(b) 


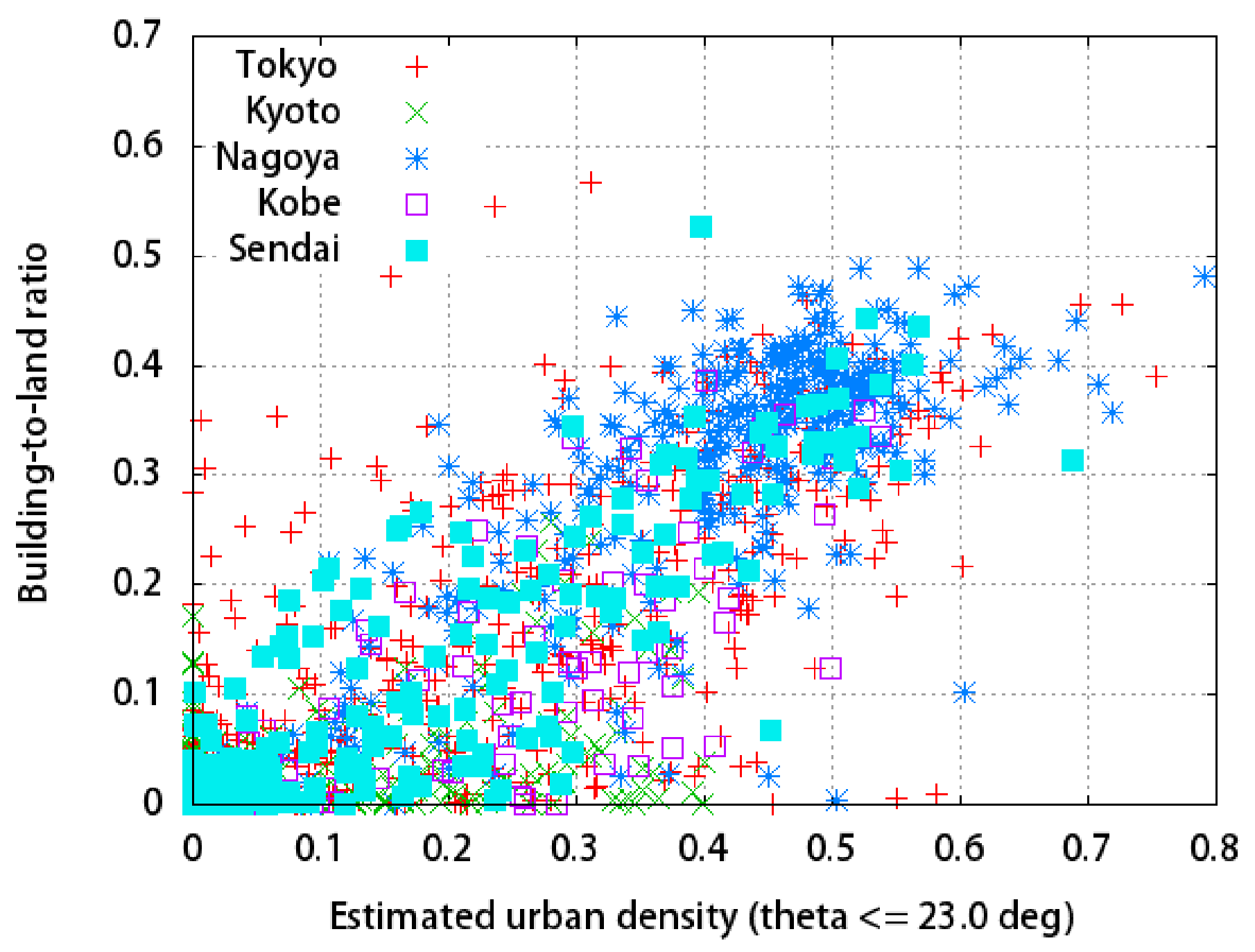

(a) 


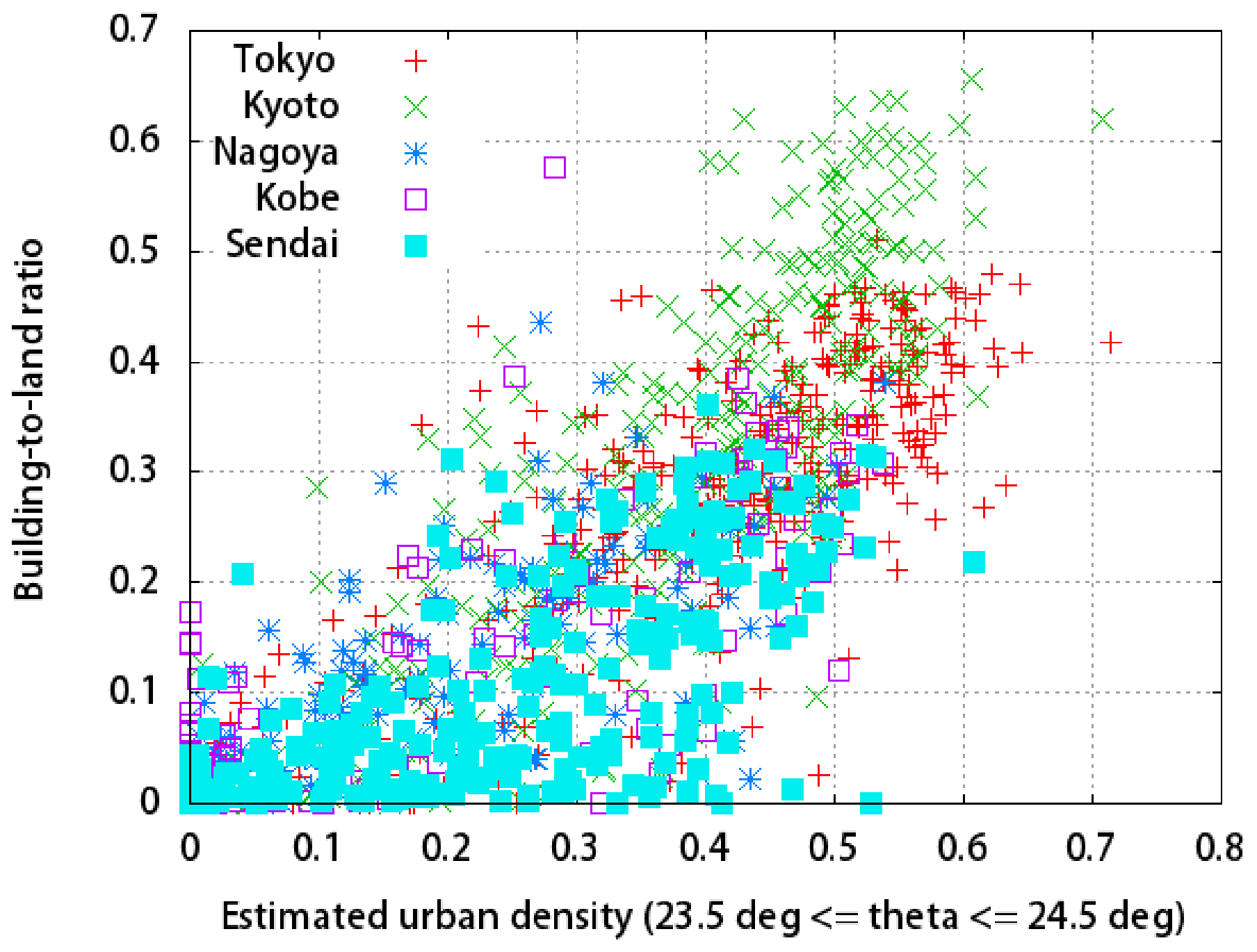

(b) 


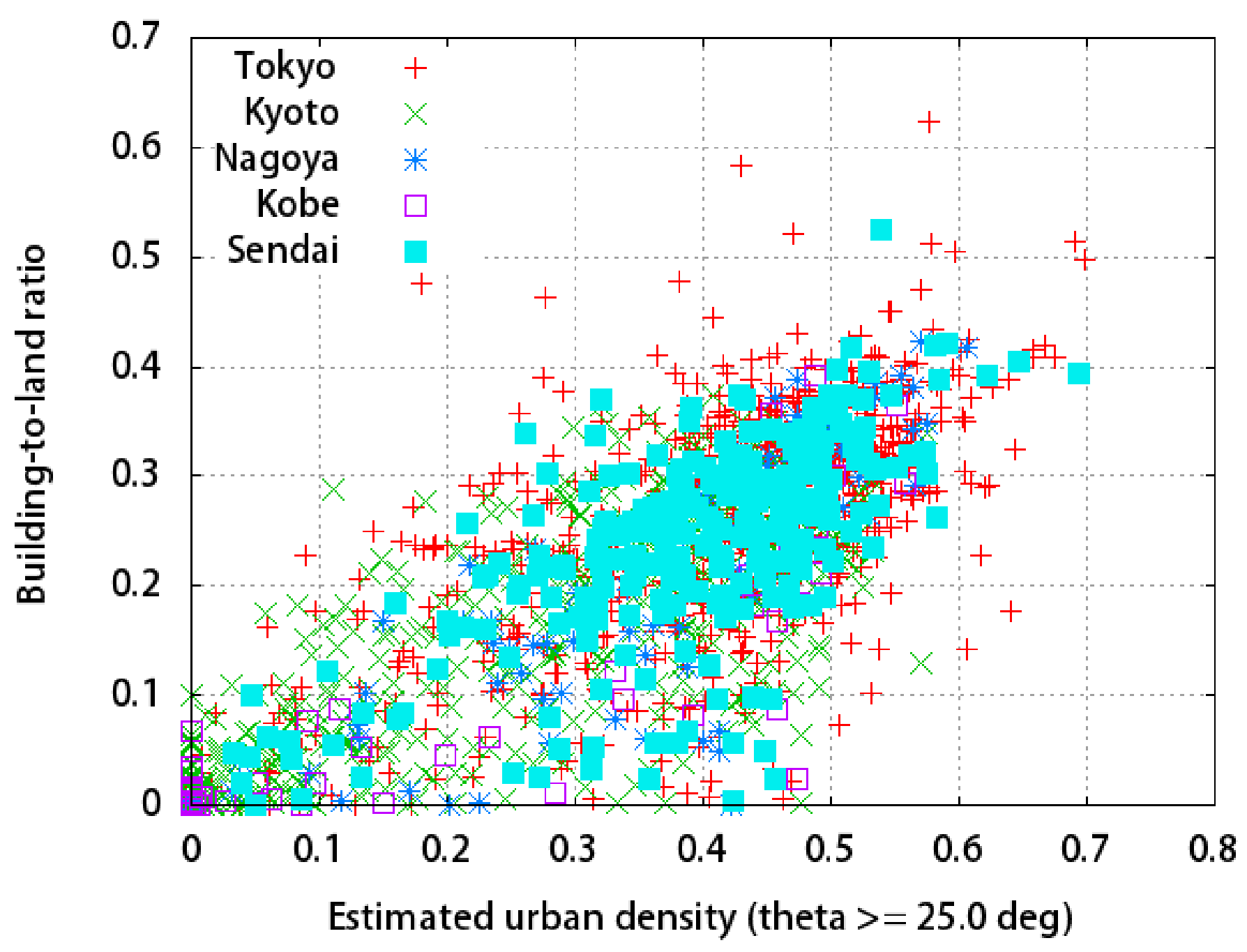

(c) 


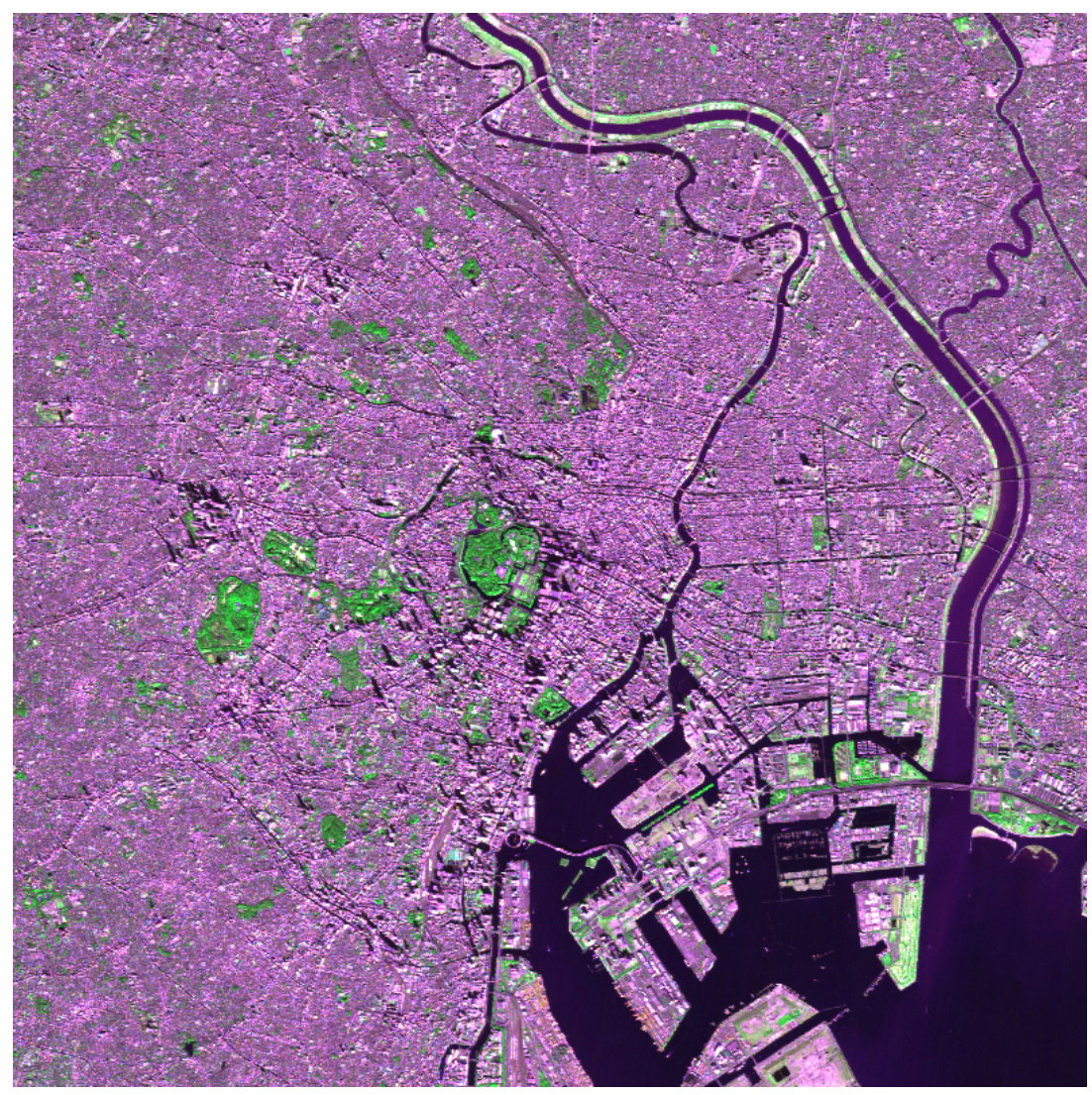

(a)

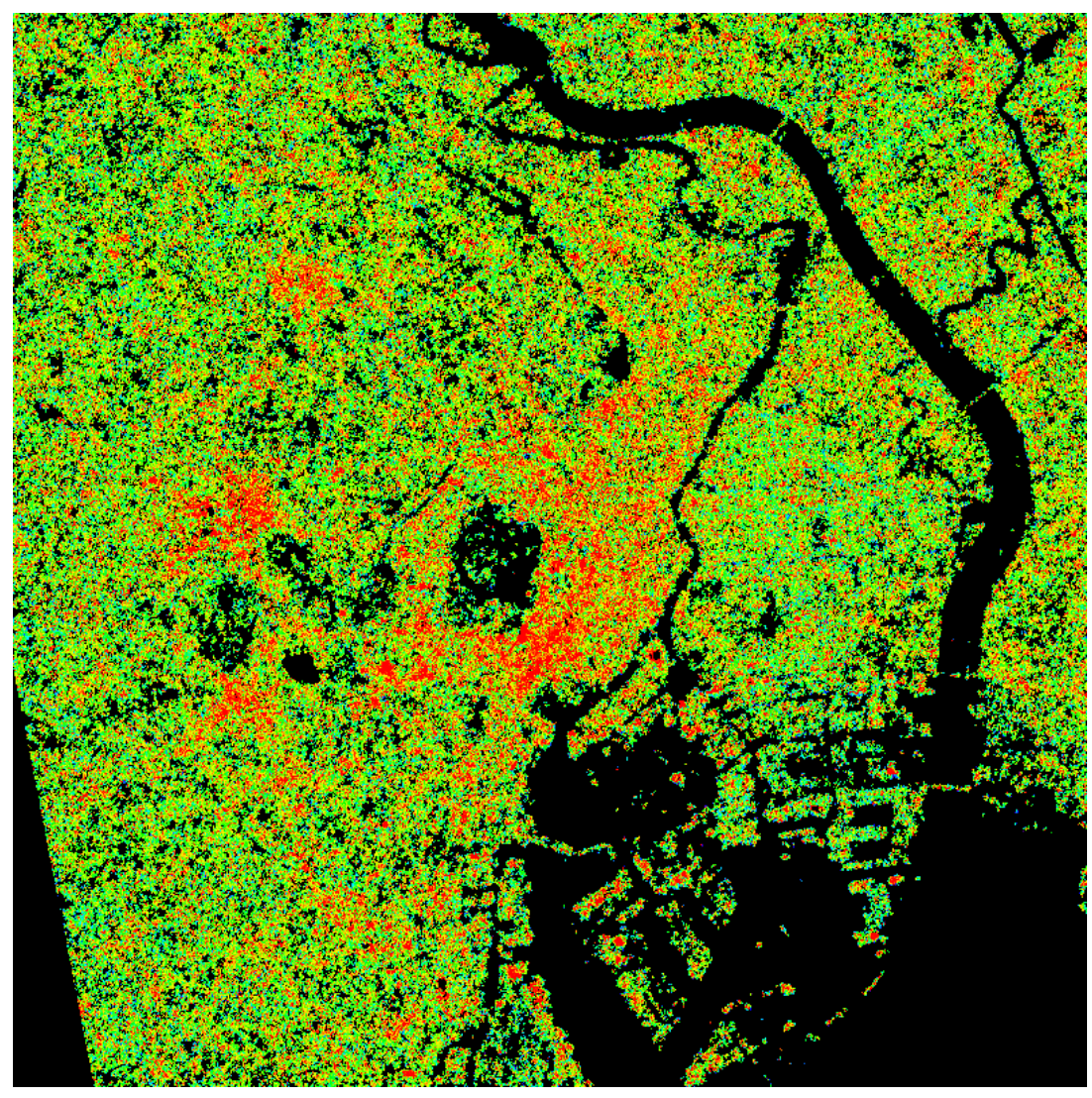

(b)
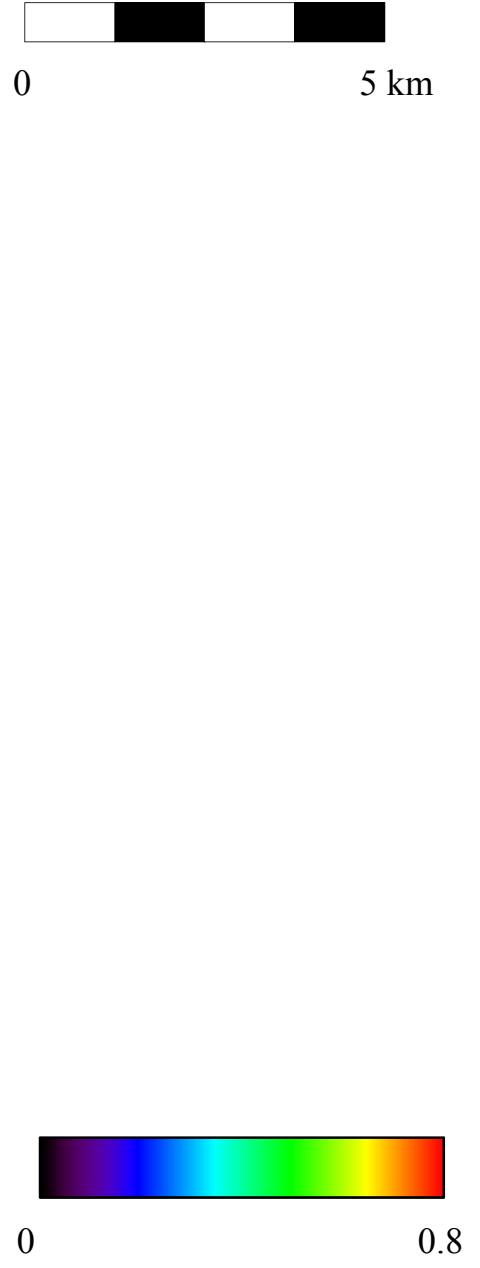


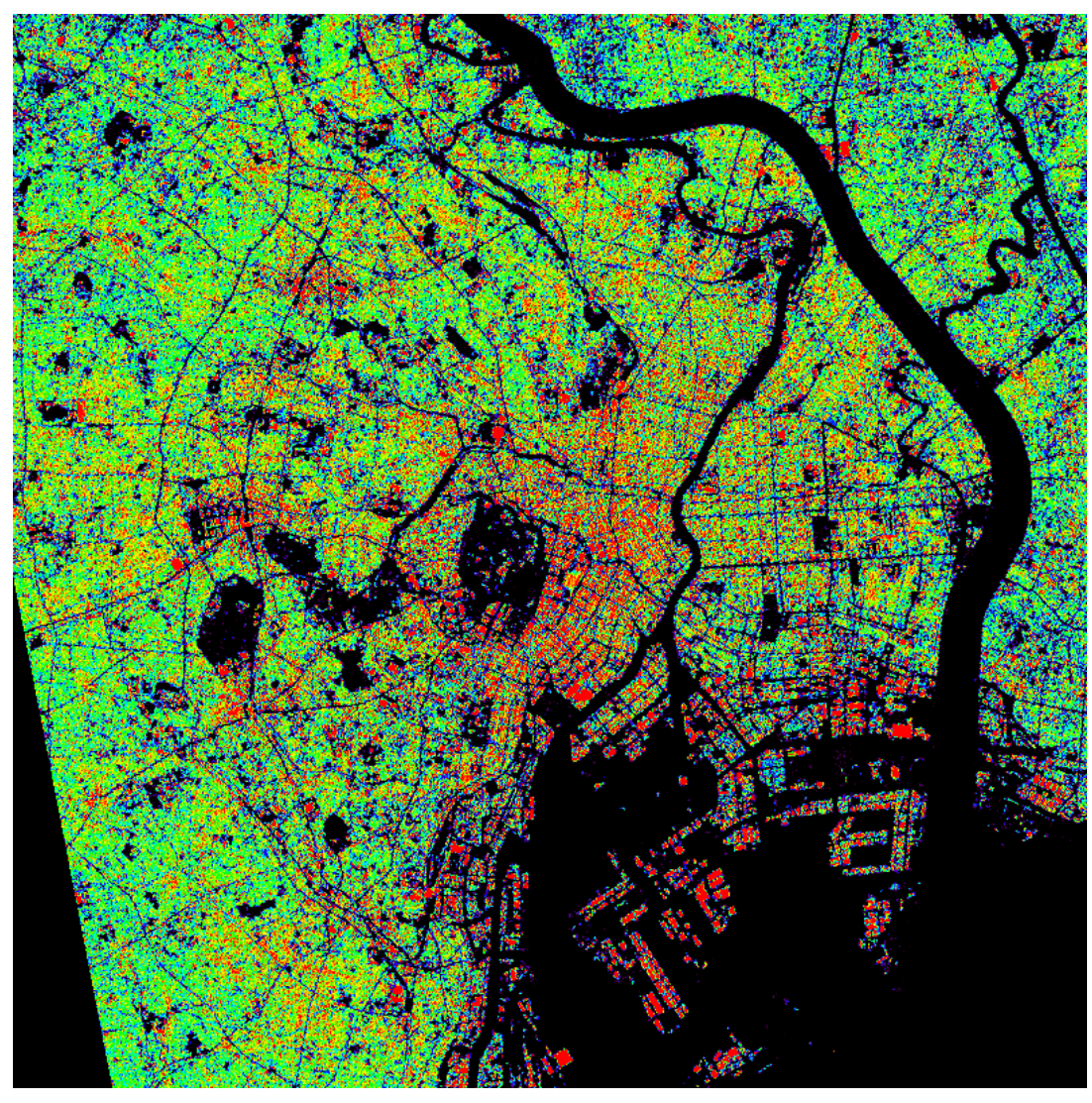

(c)

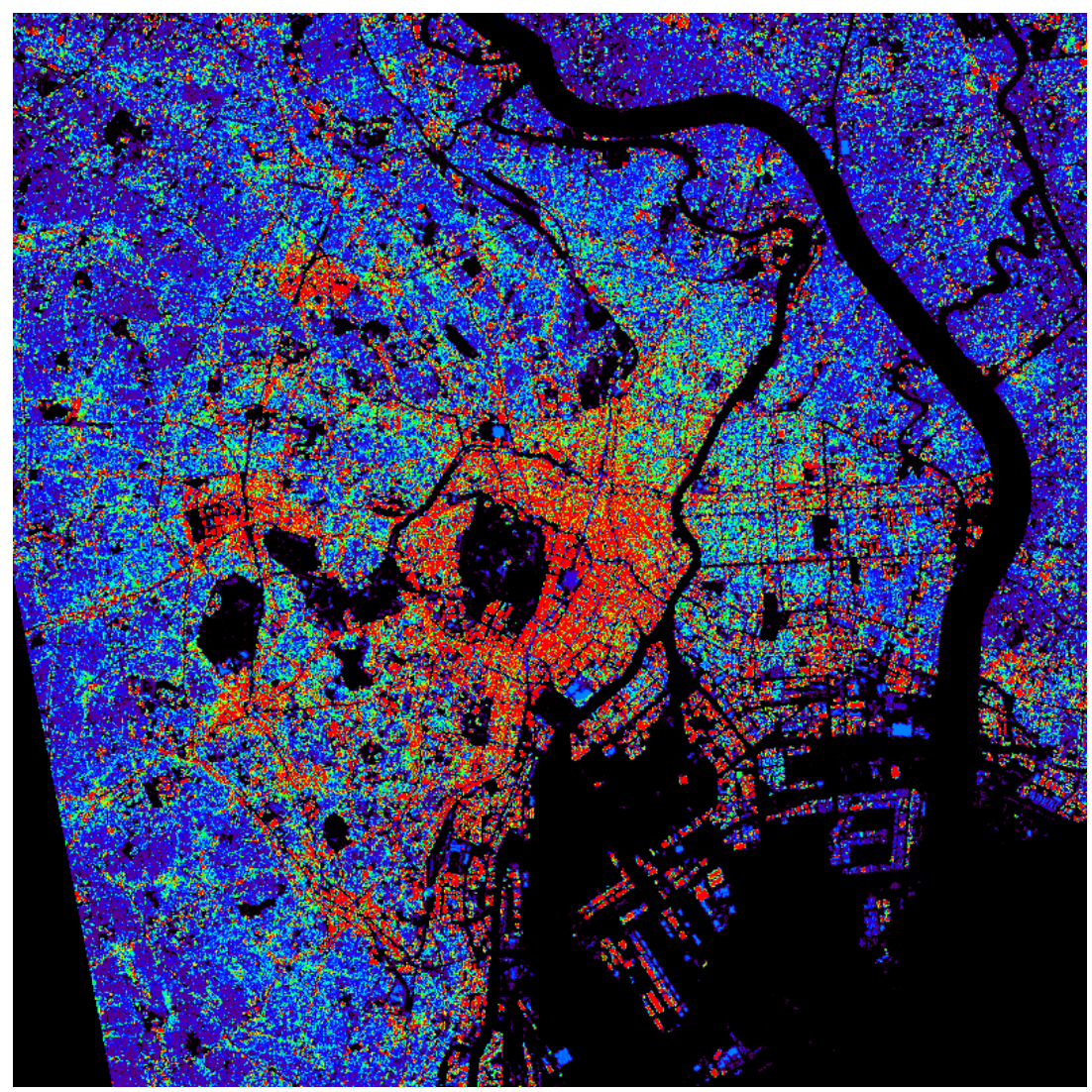

(d)
0

0.8

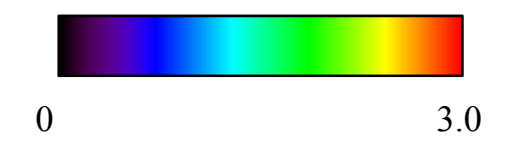




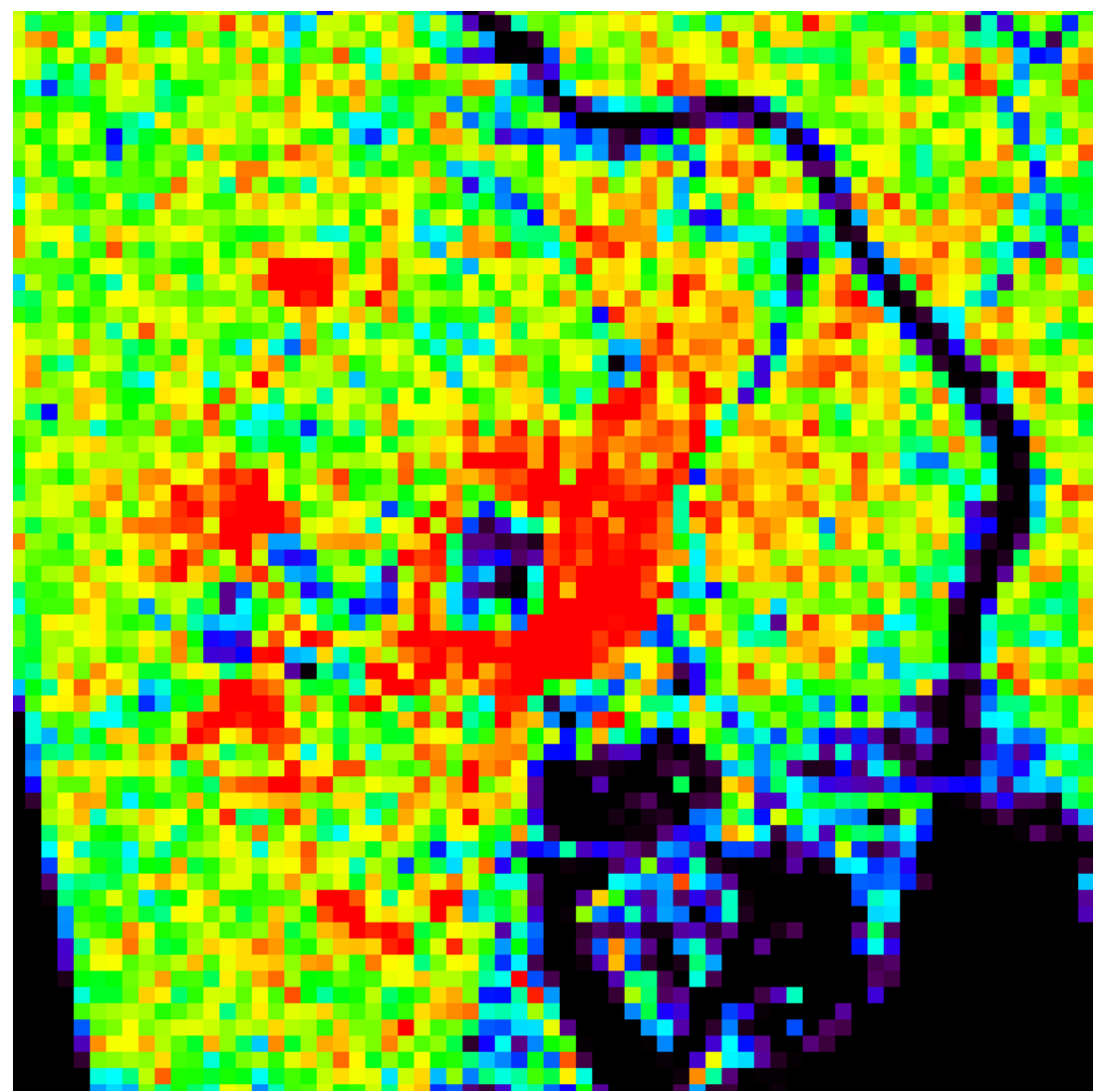

(e)

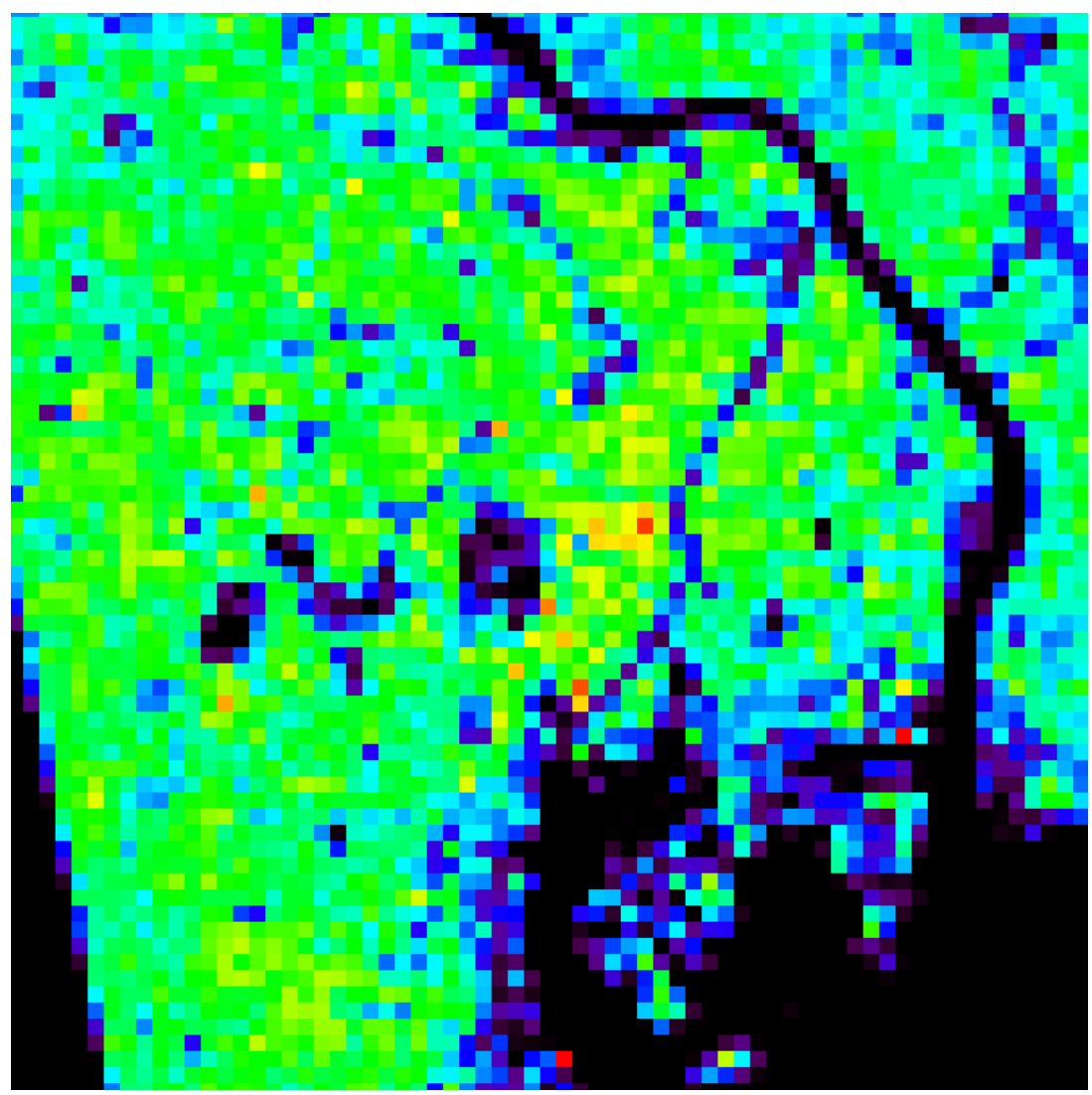

(f)

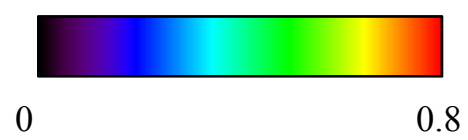

0.8 

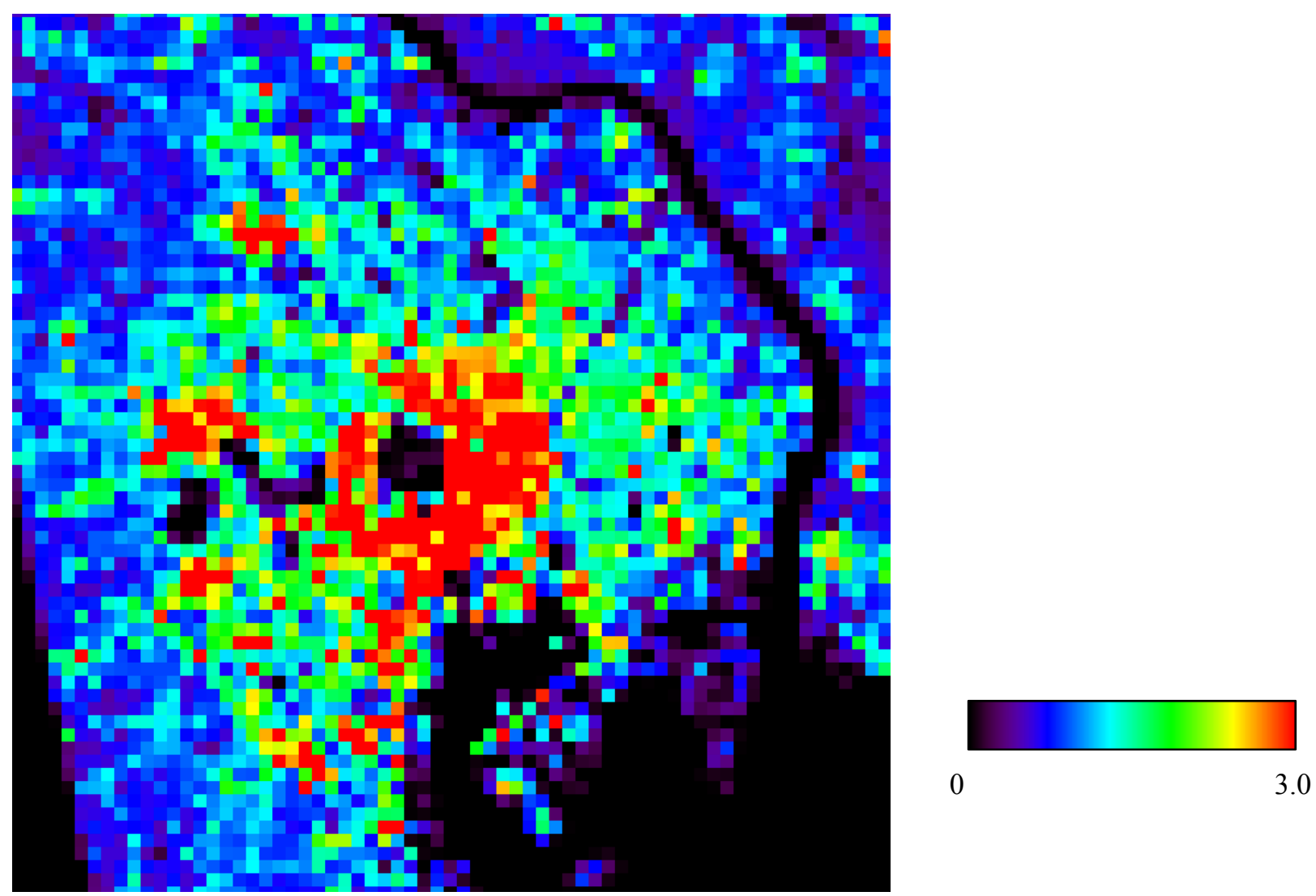

(g) 


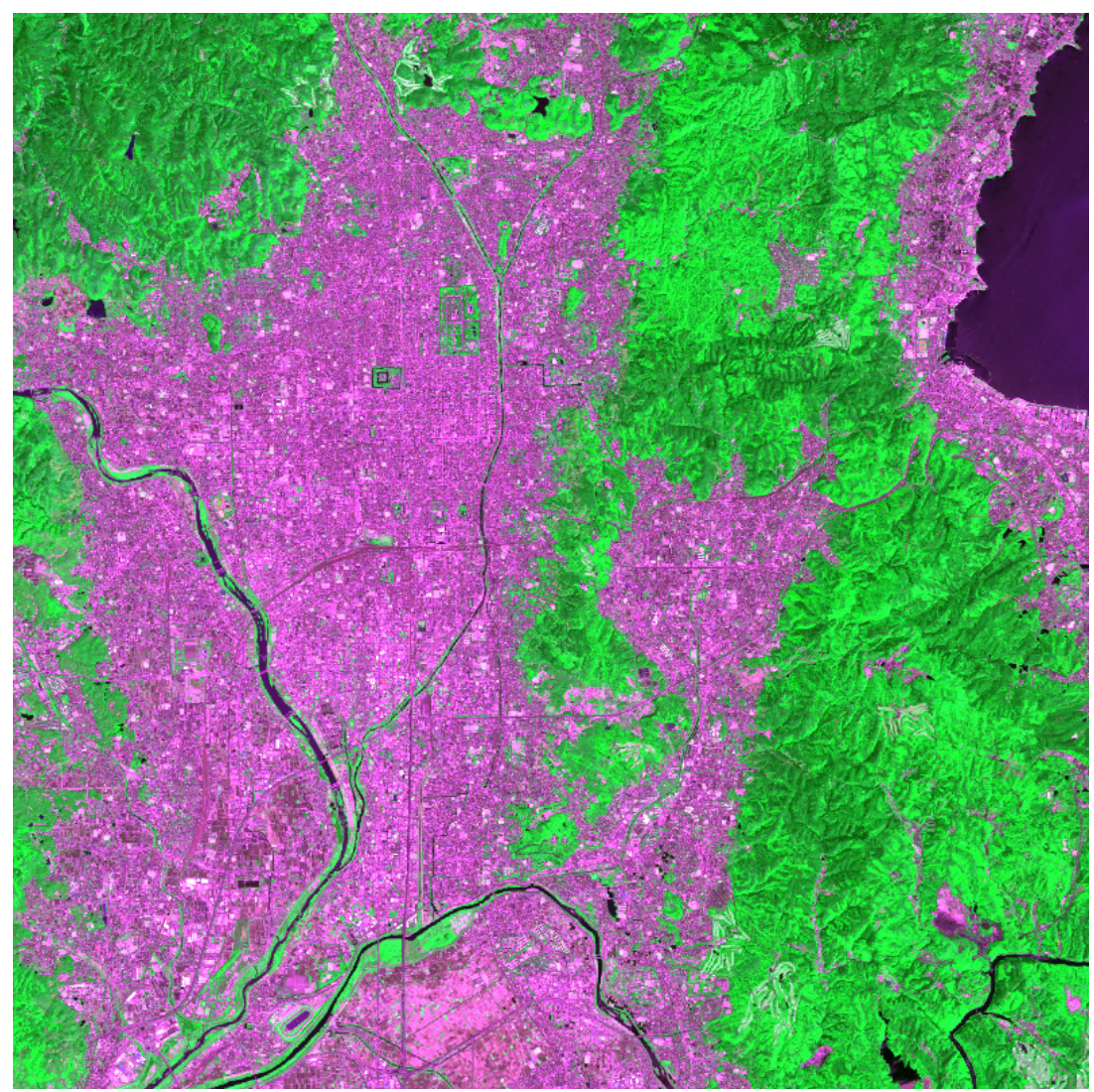

(a)

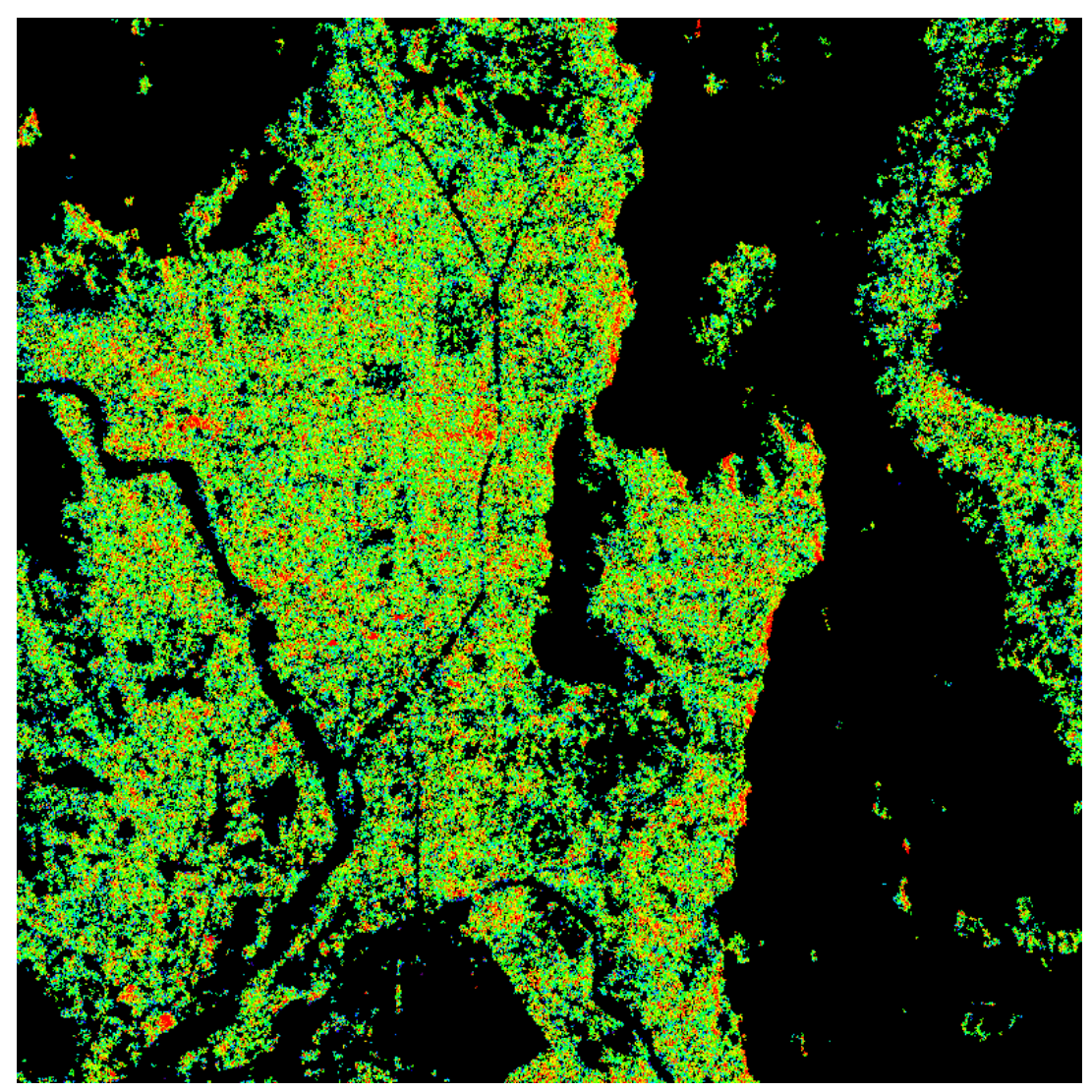

(b)
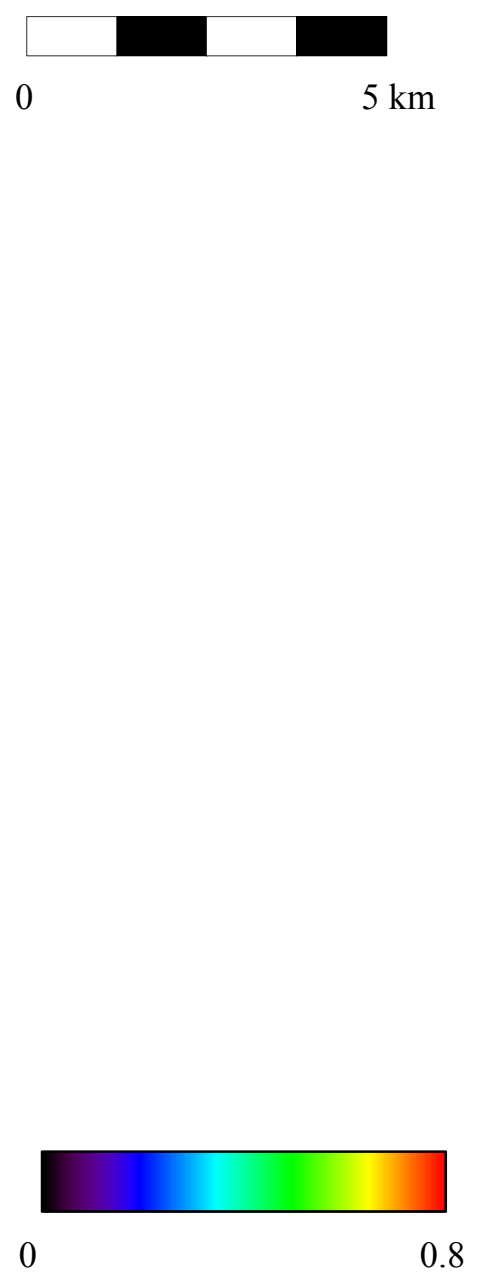

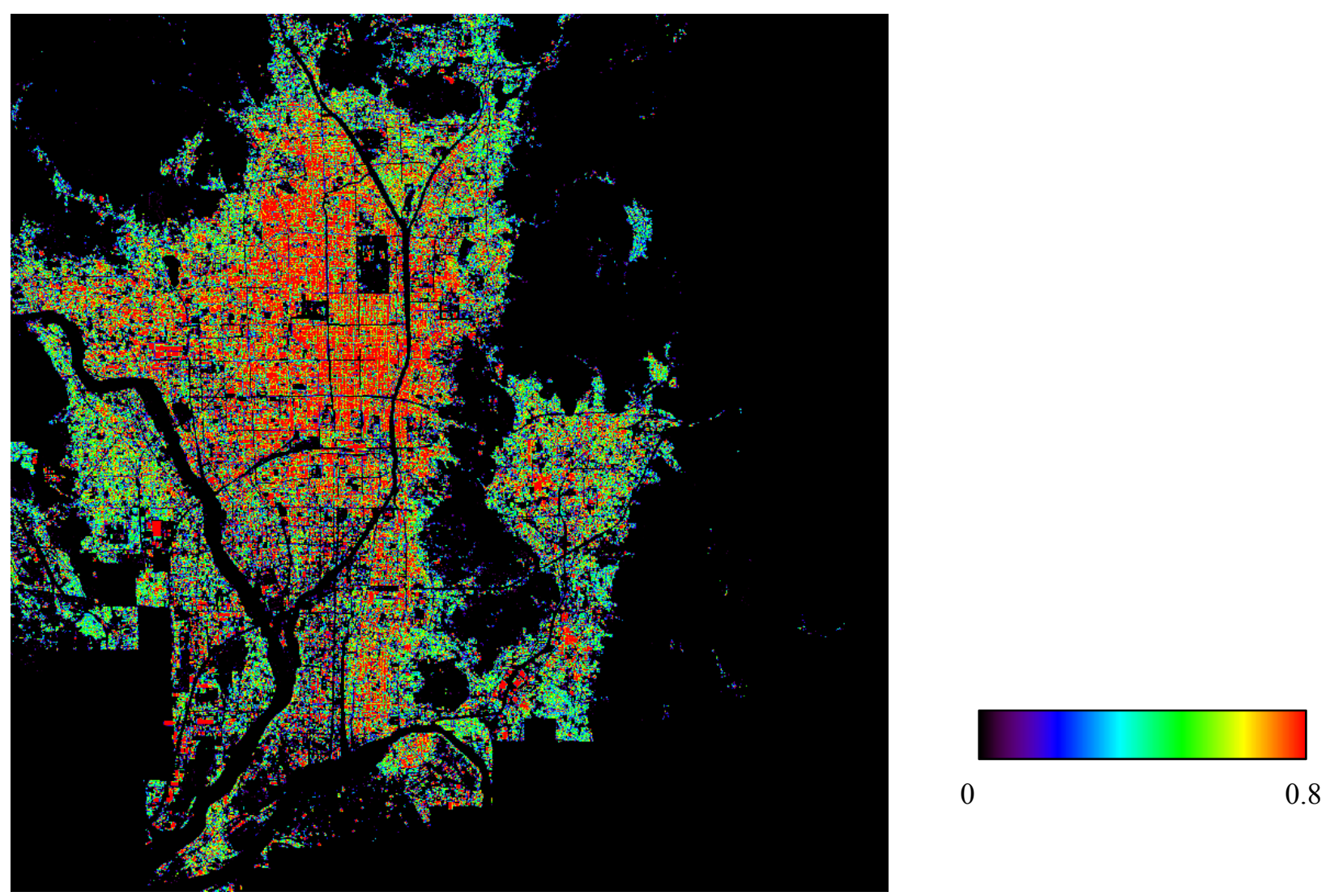

(c)
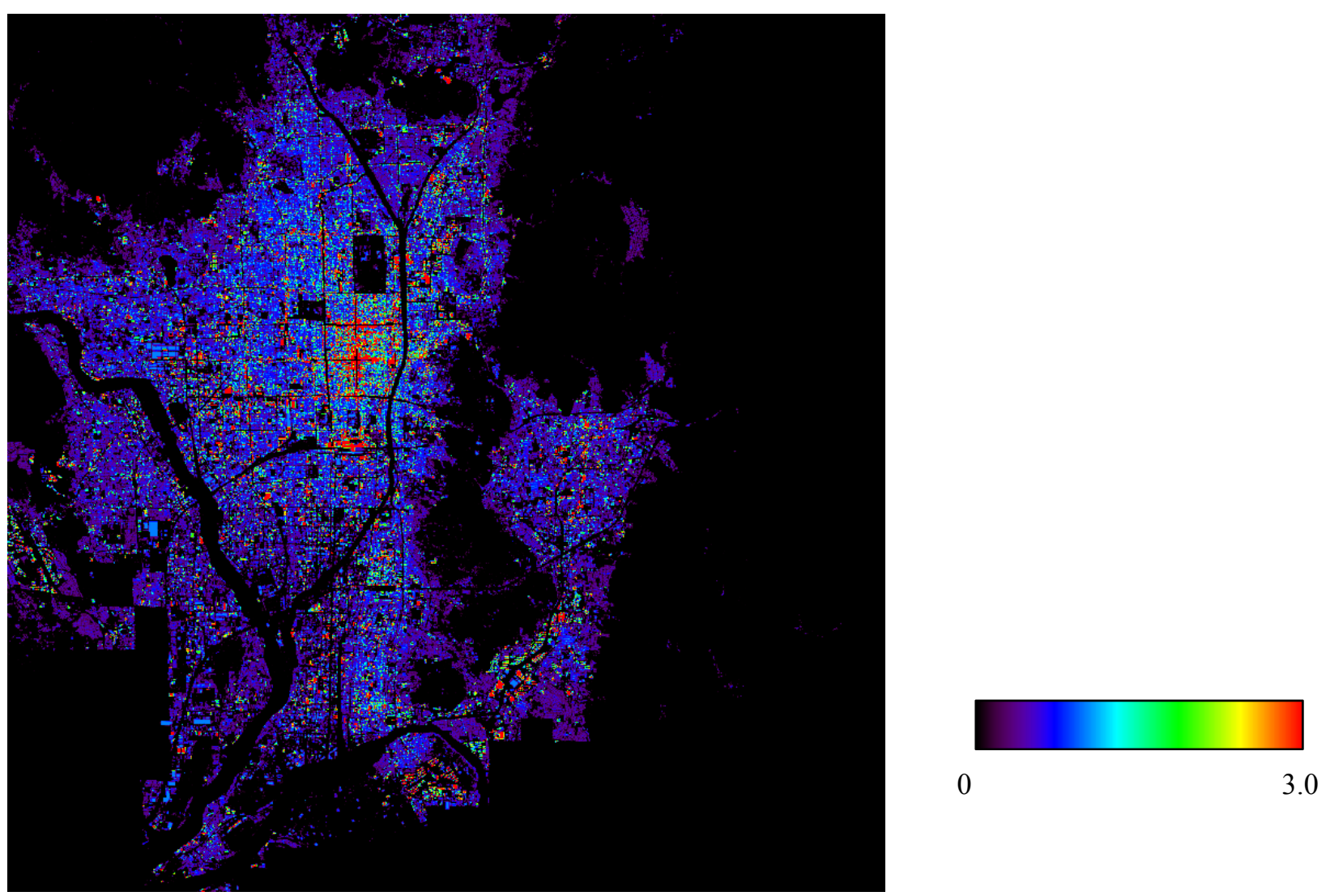

(d) 


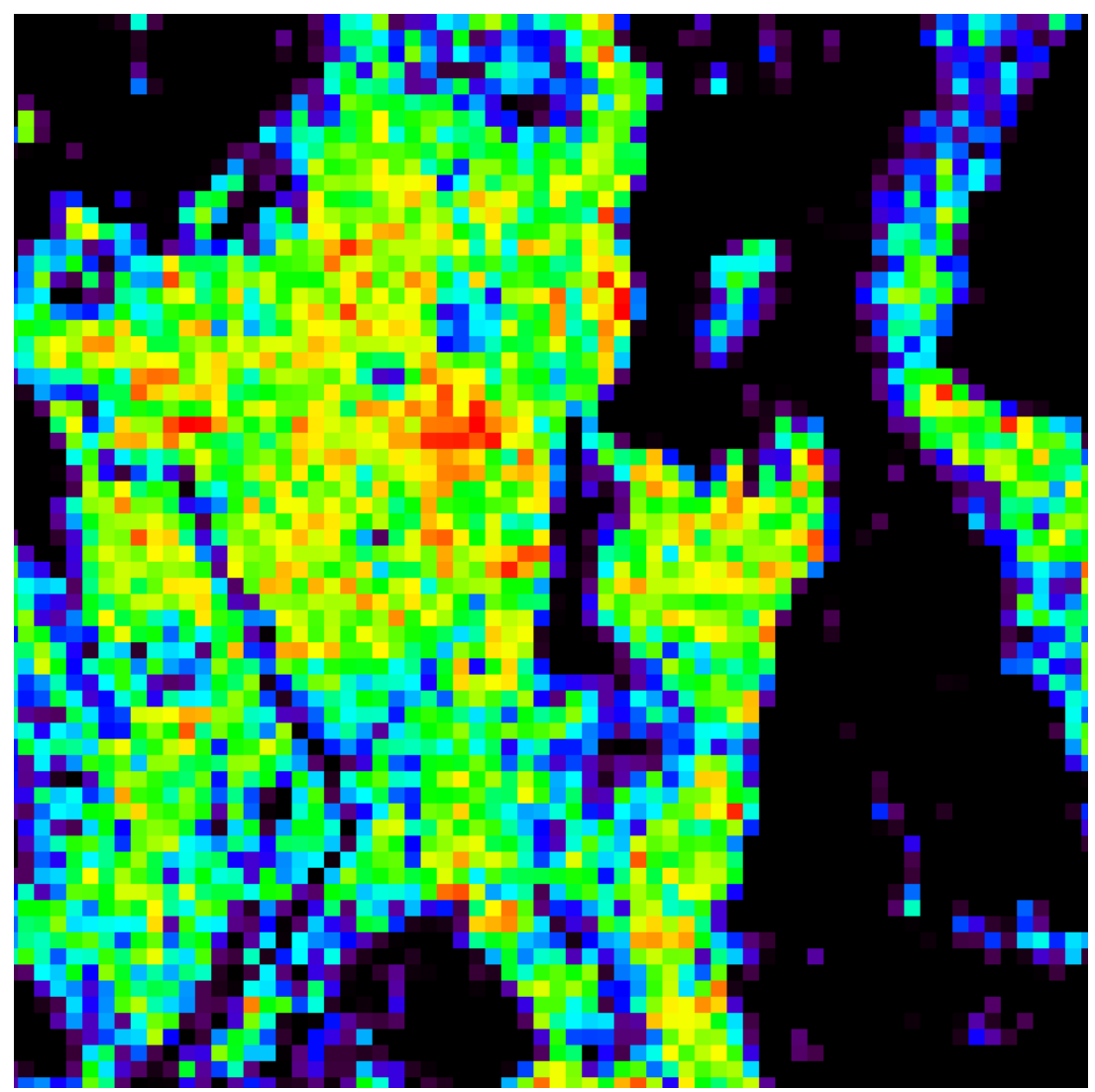

(e)

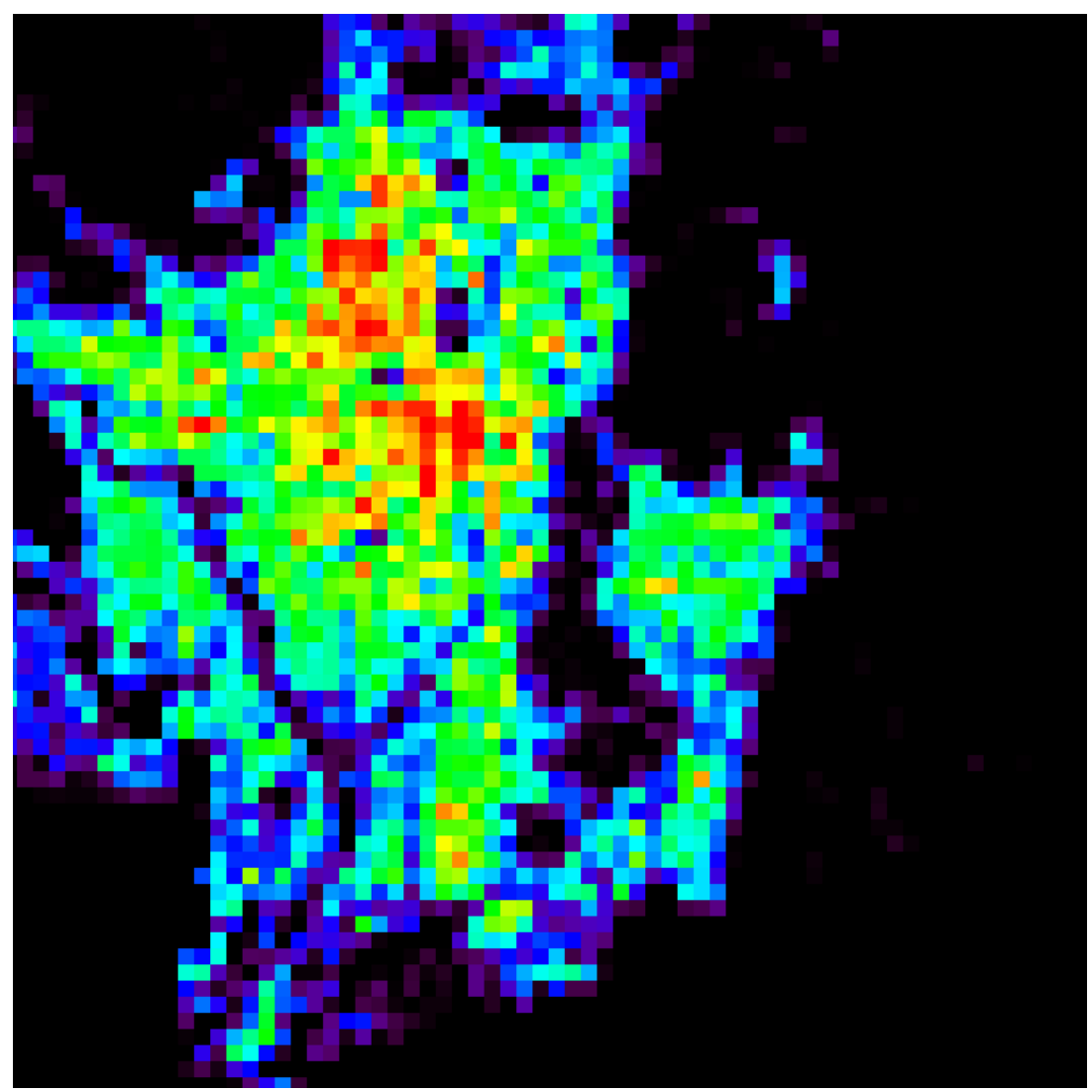

(f)
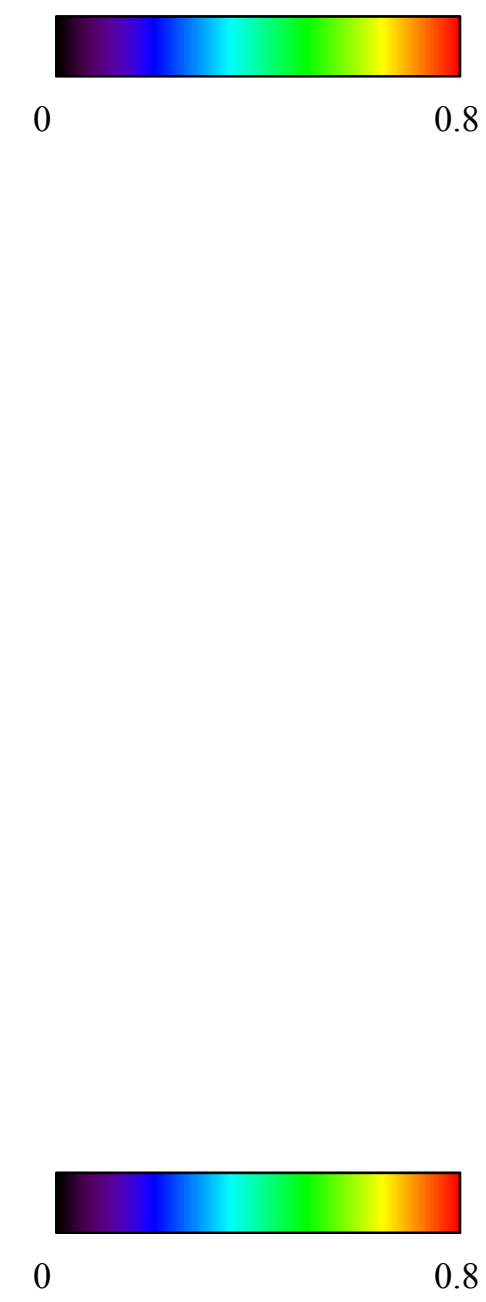

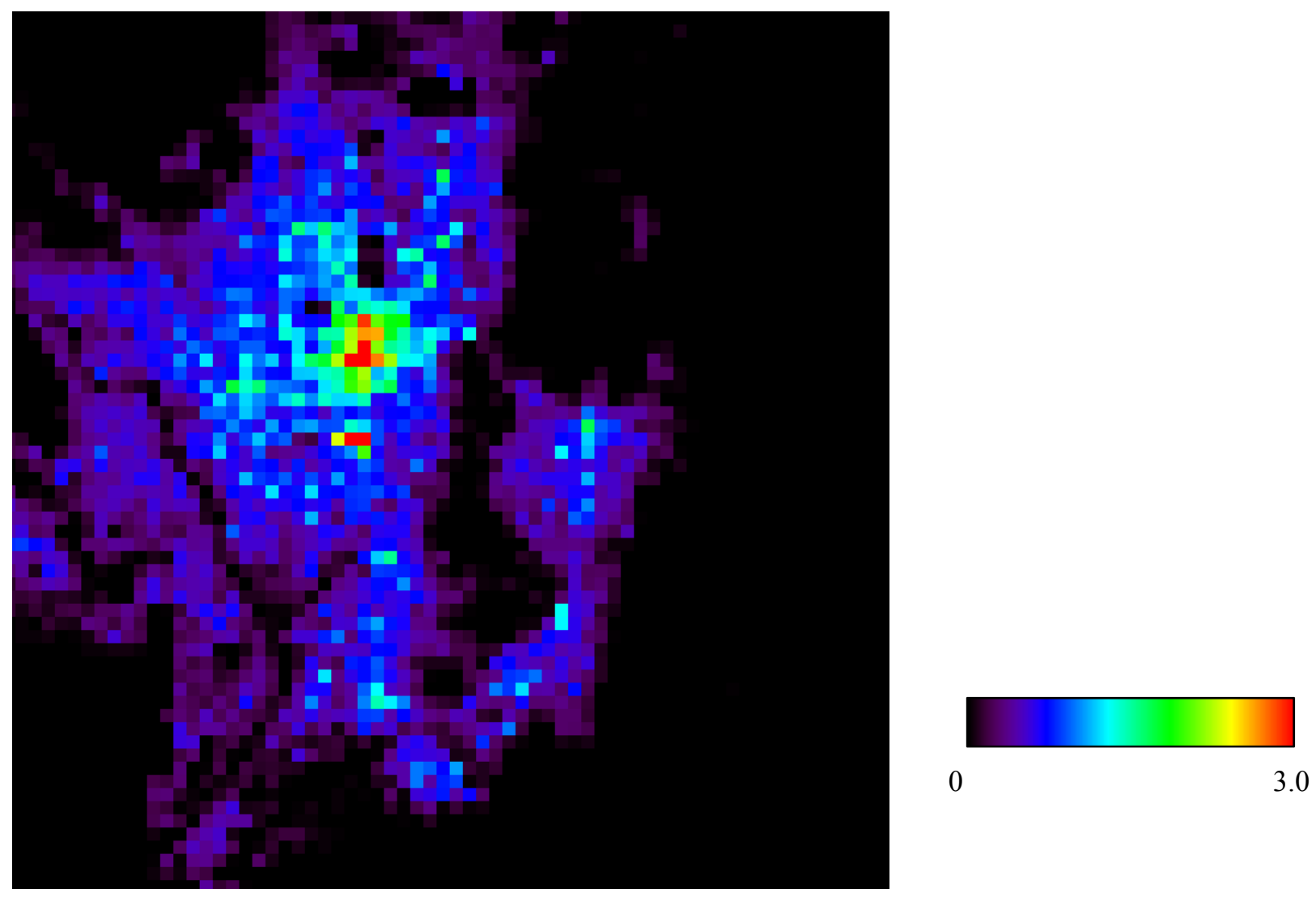

(g) 


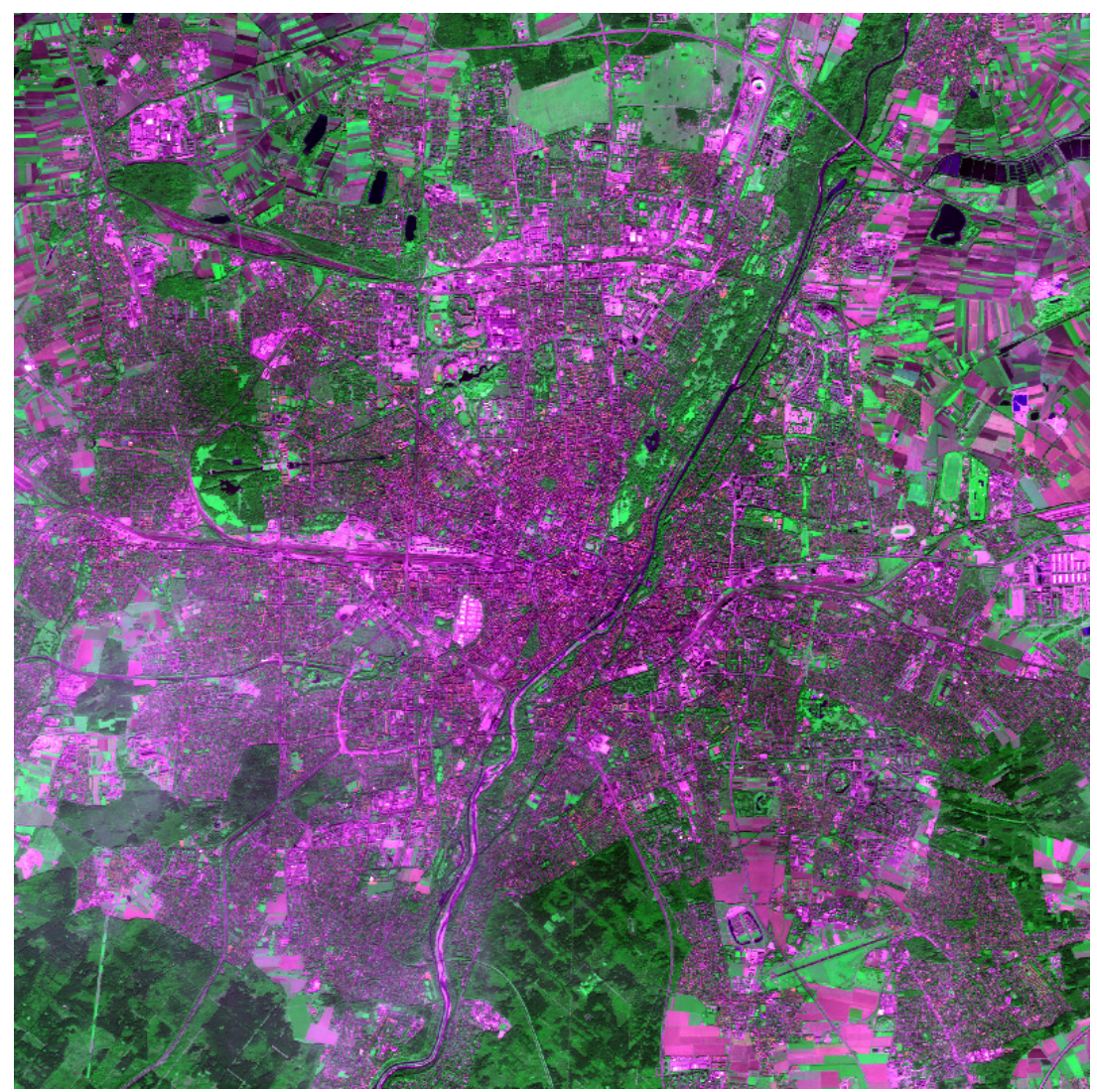

(a)

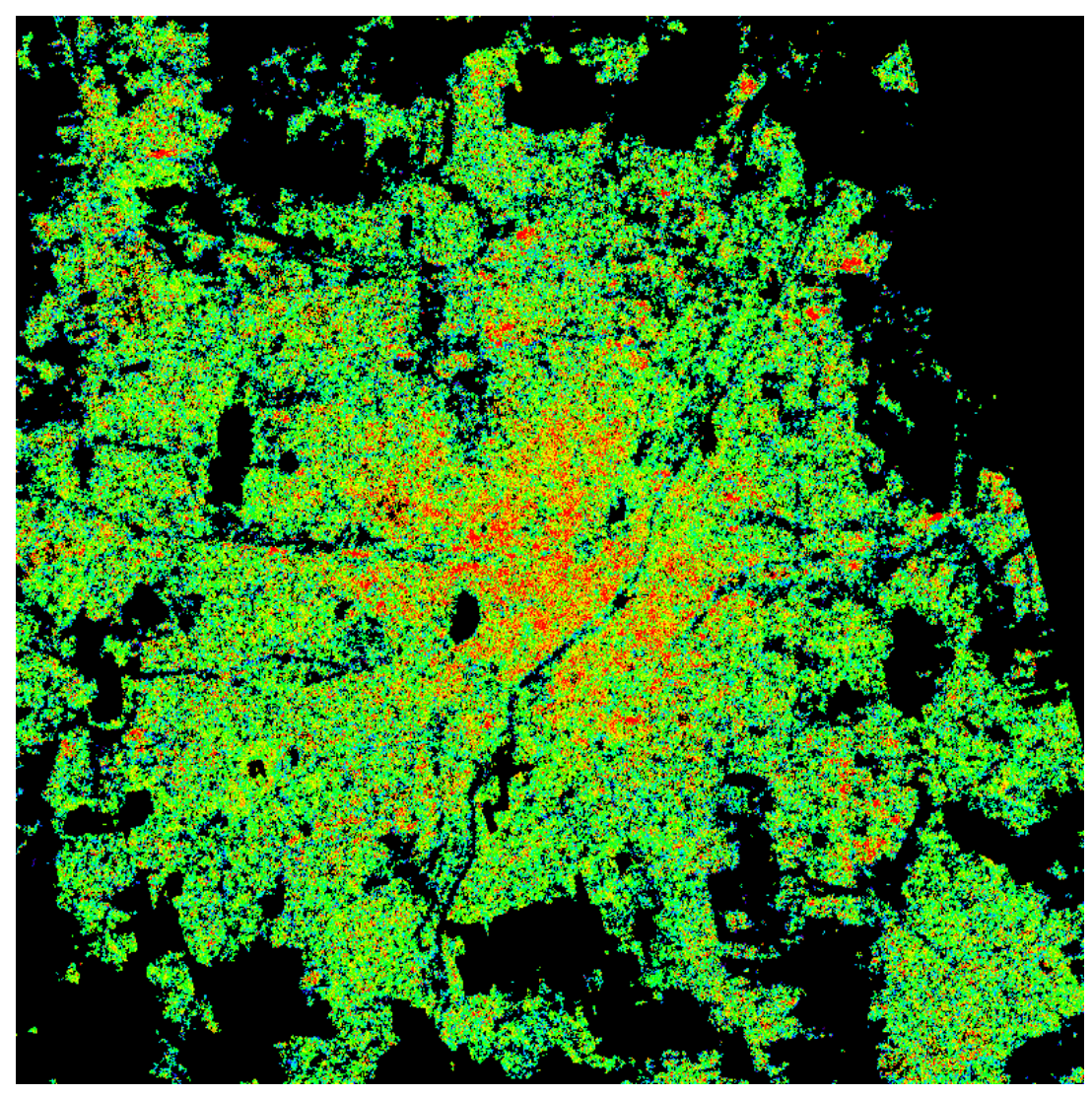

(b)
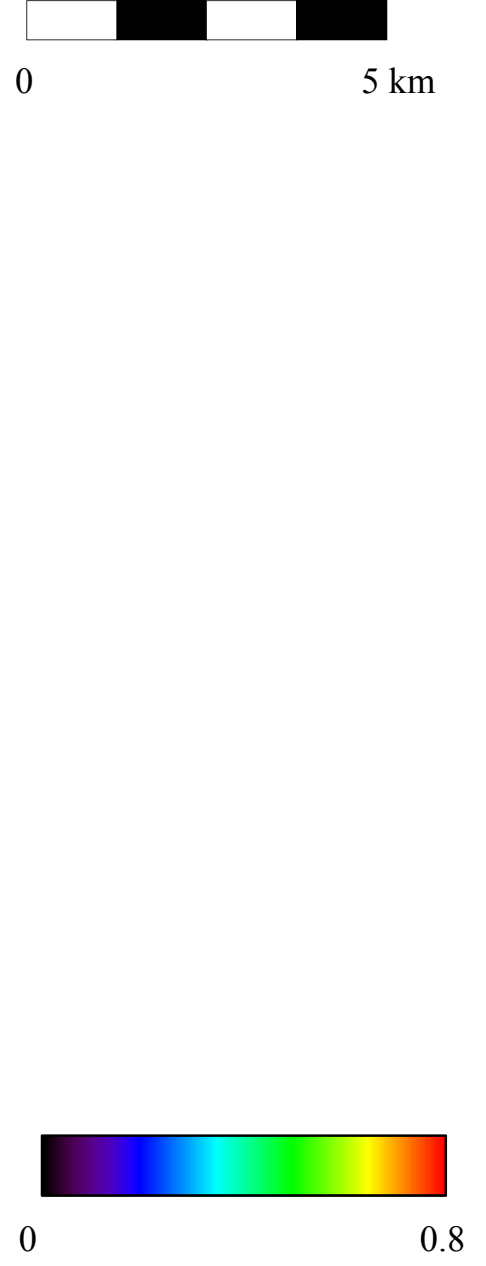

0

0.8 


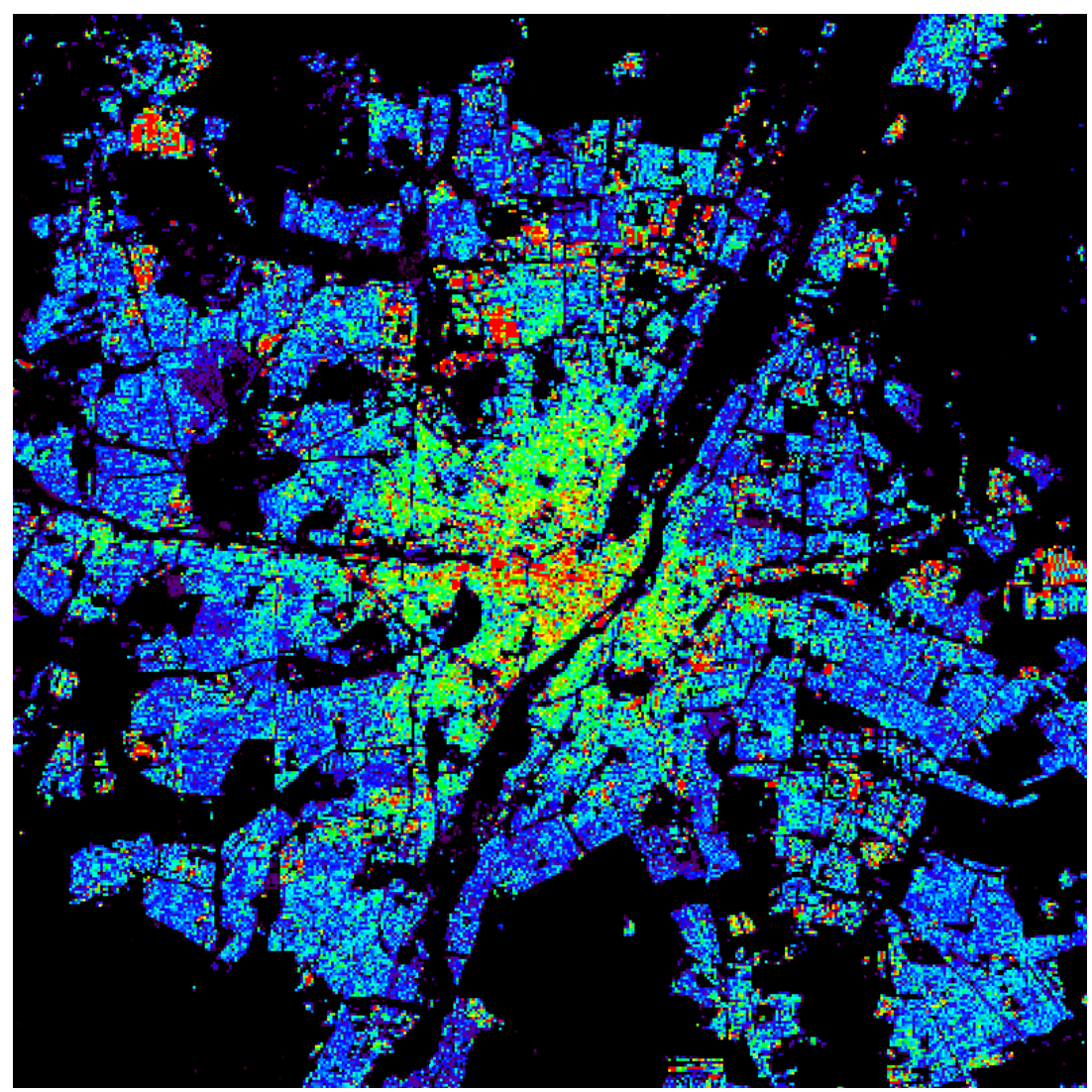

(c)

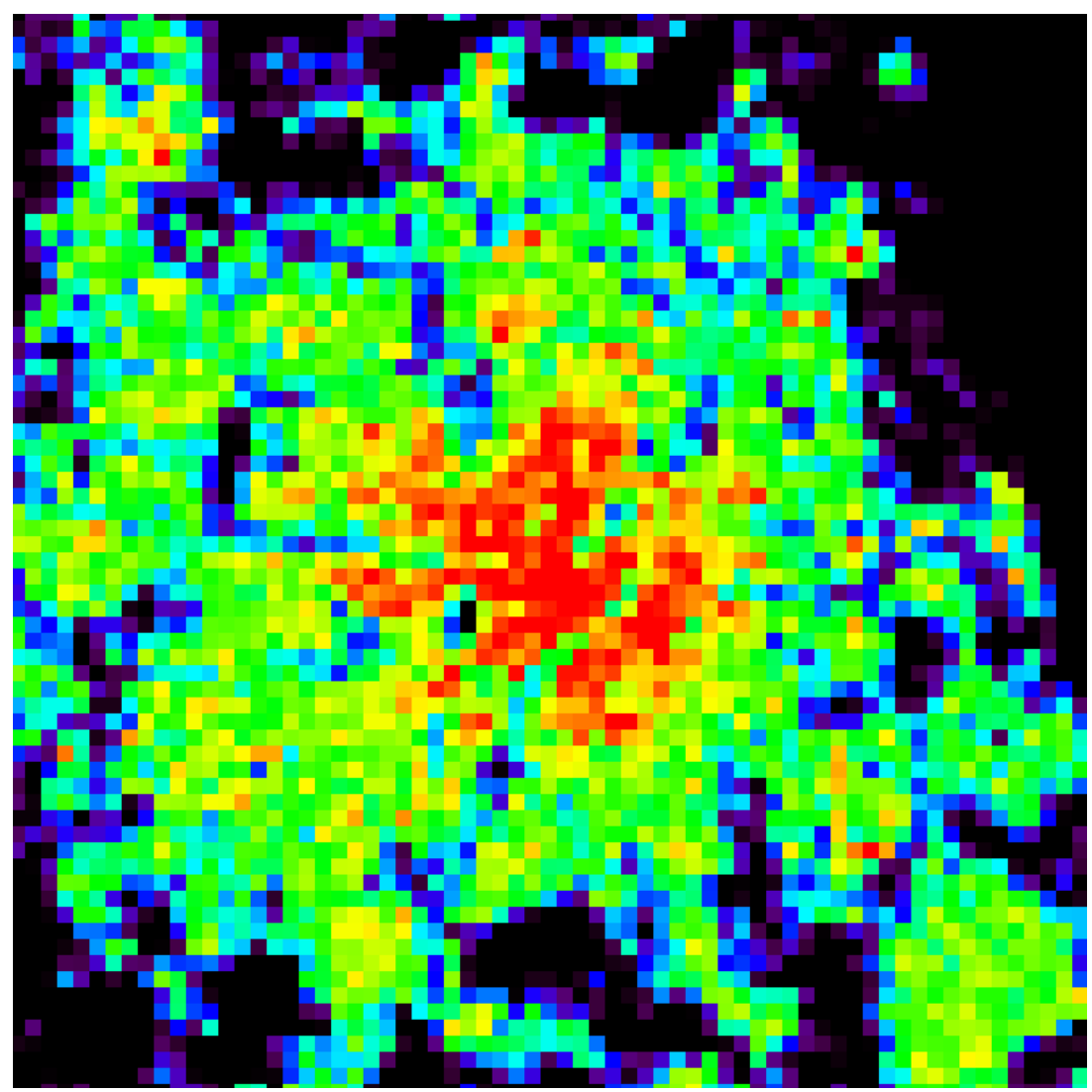

(d)
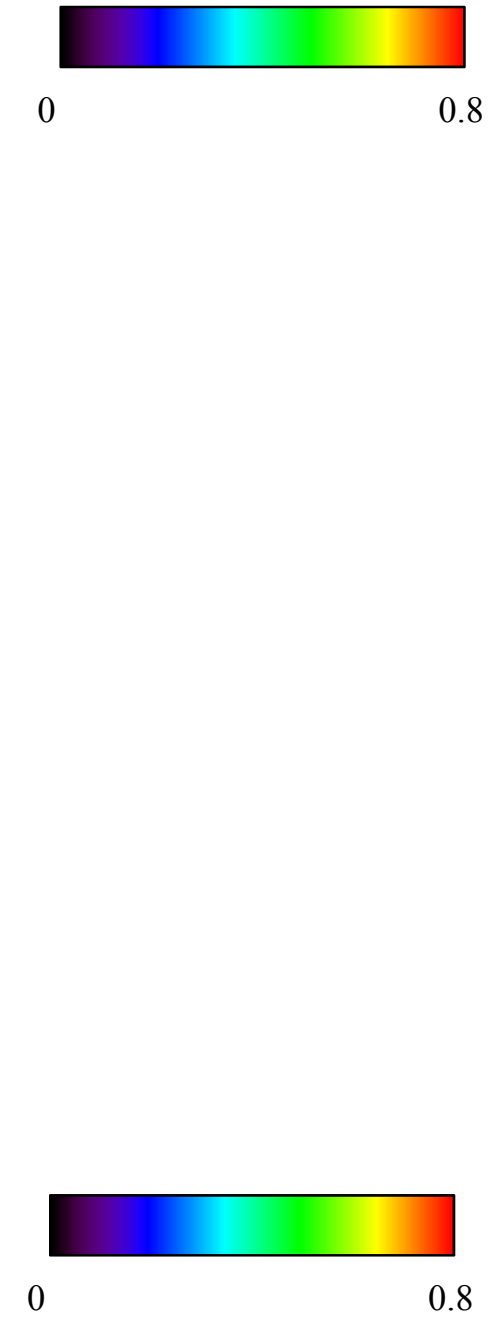

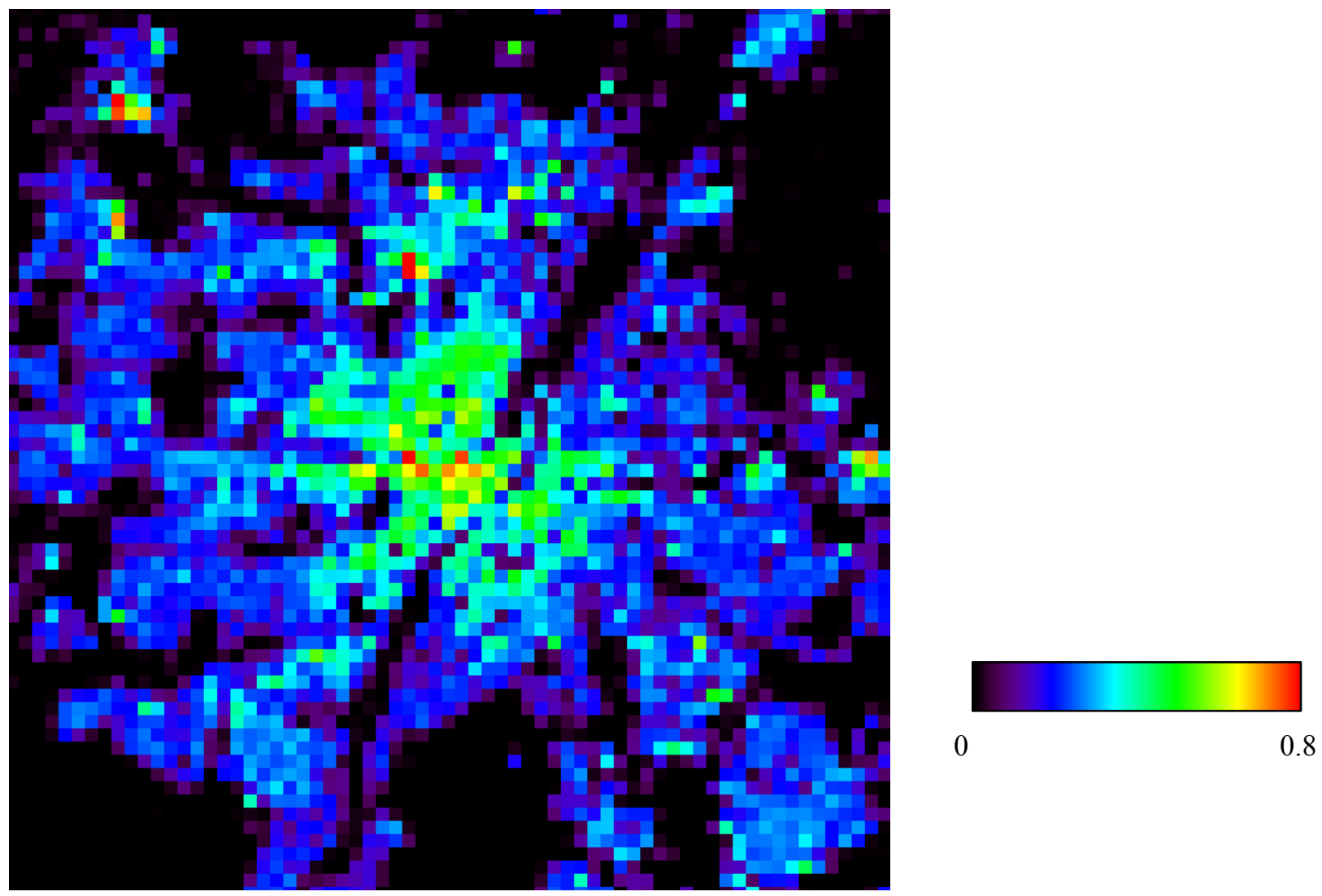

(e) 


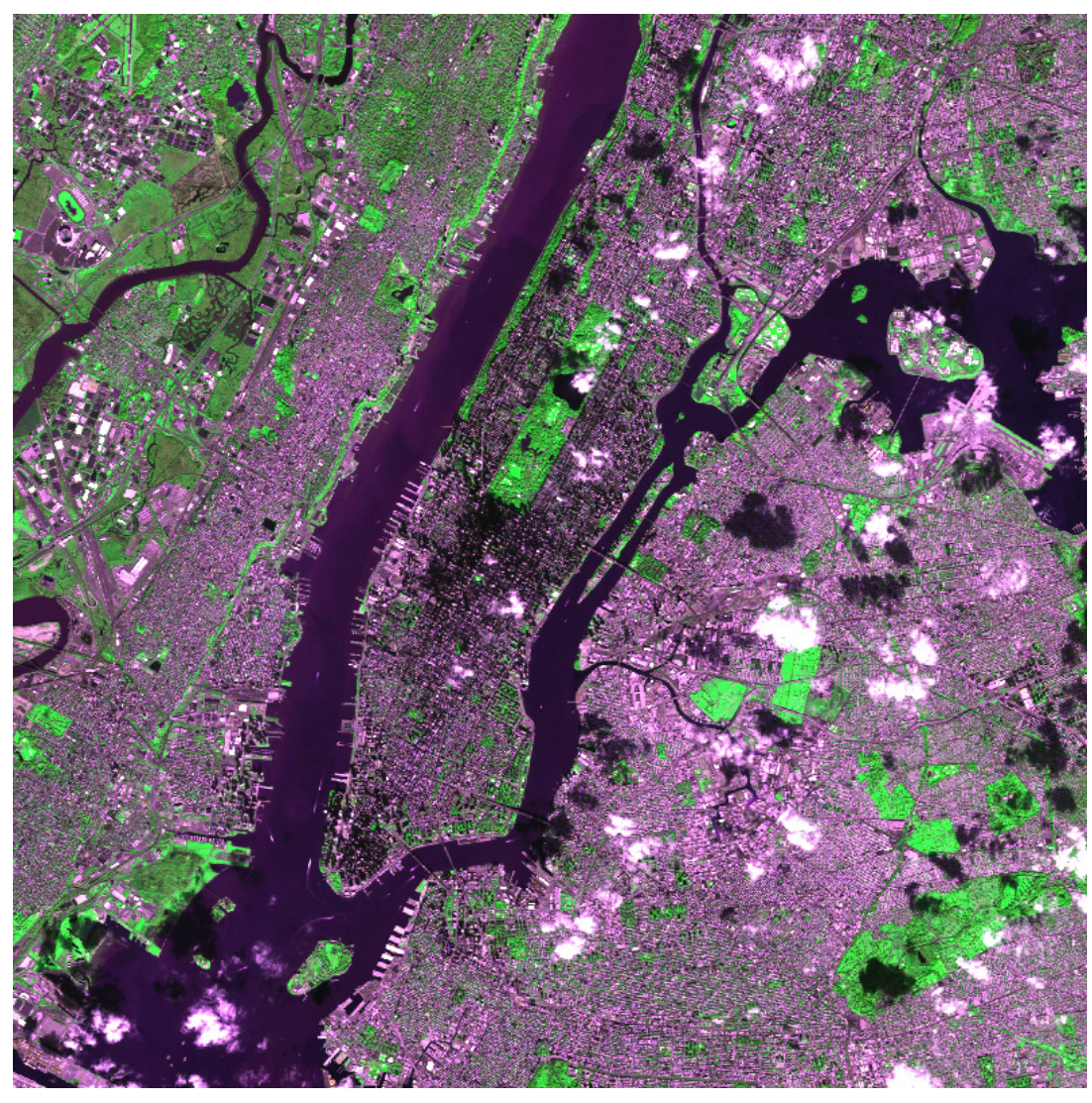

(a)

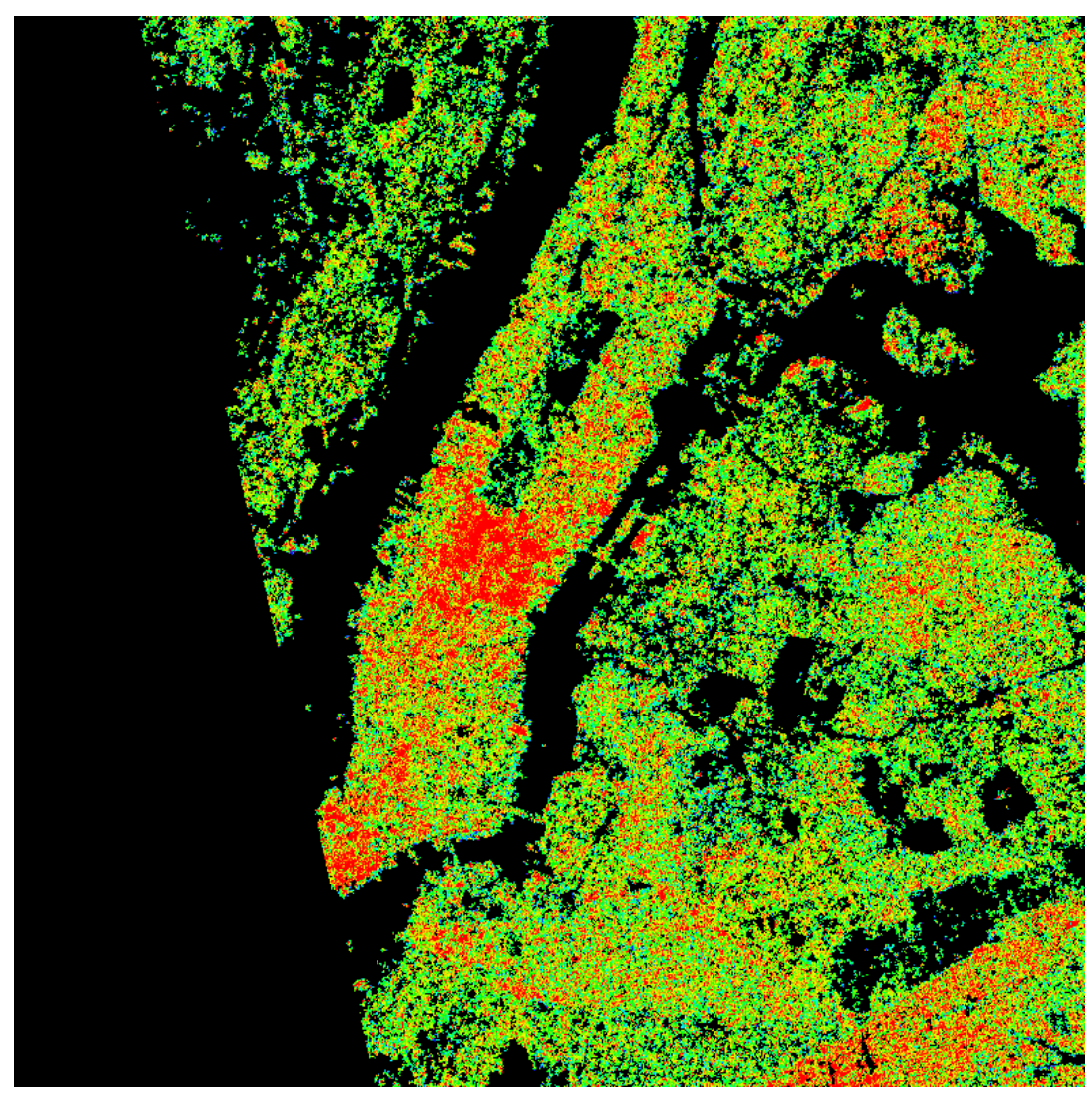

(b)
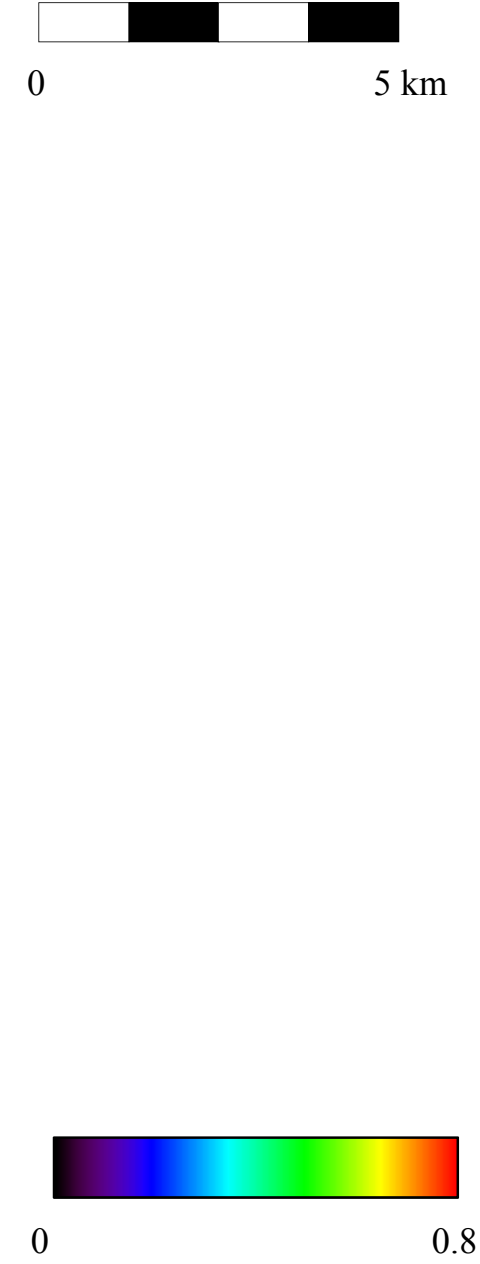

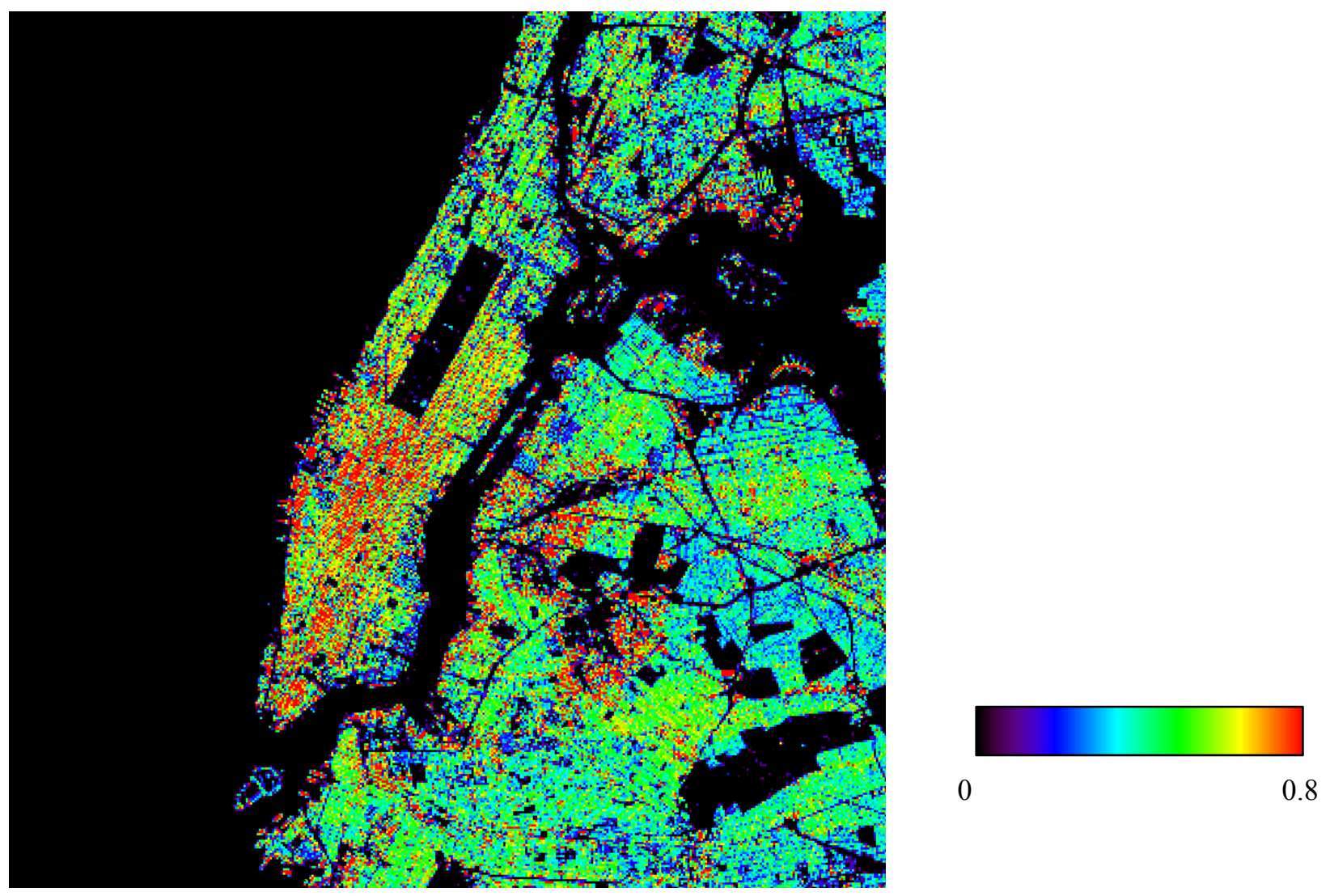

(c)
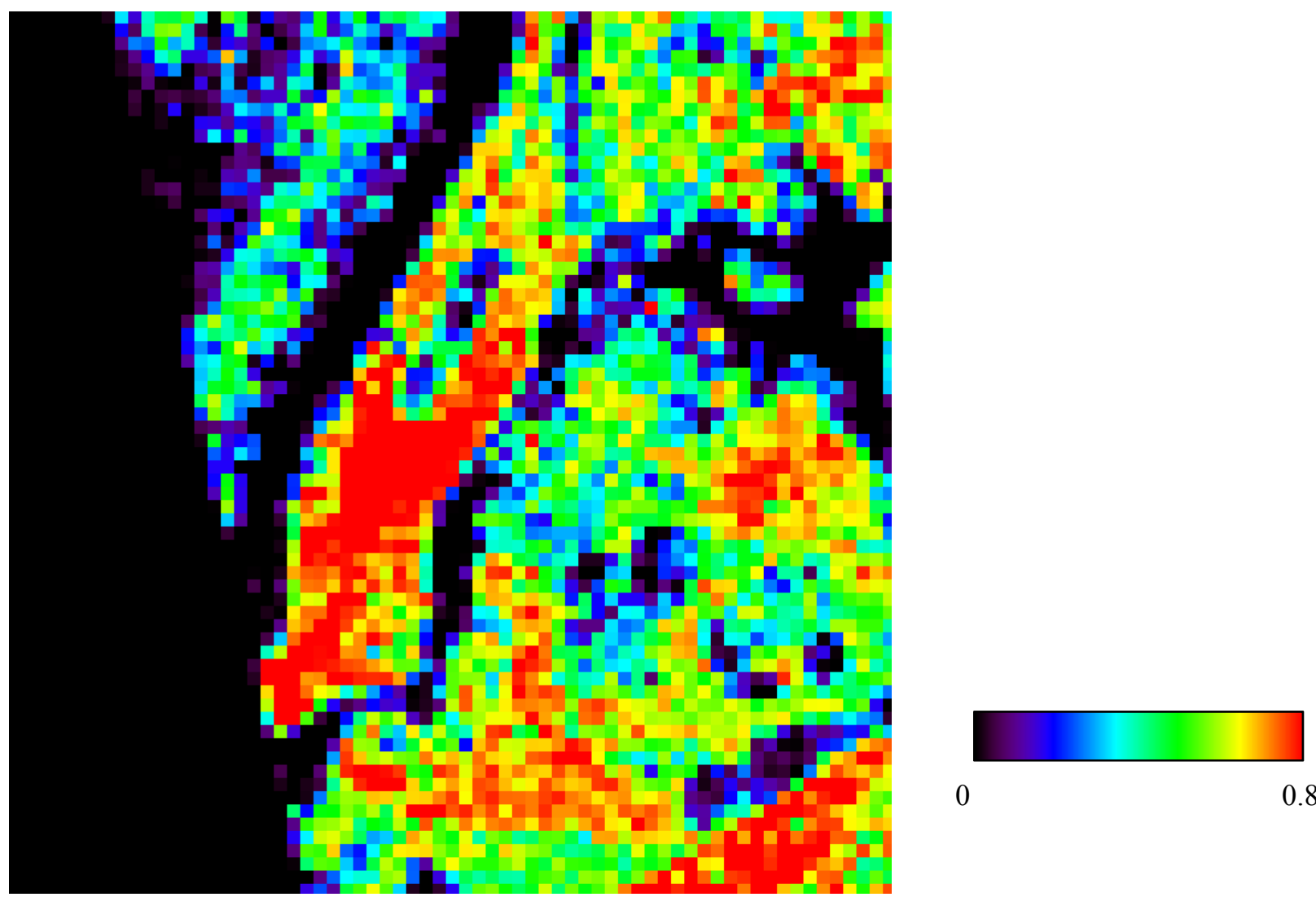

(d) 

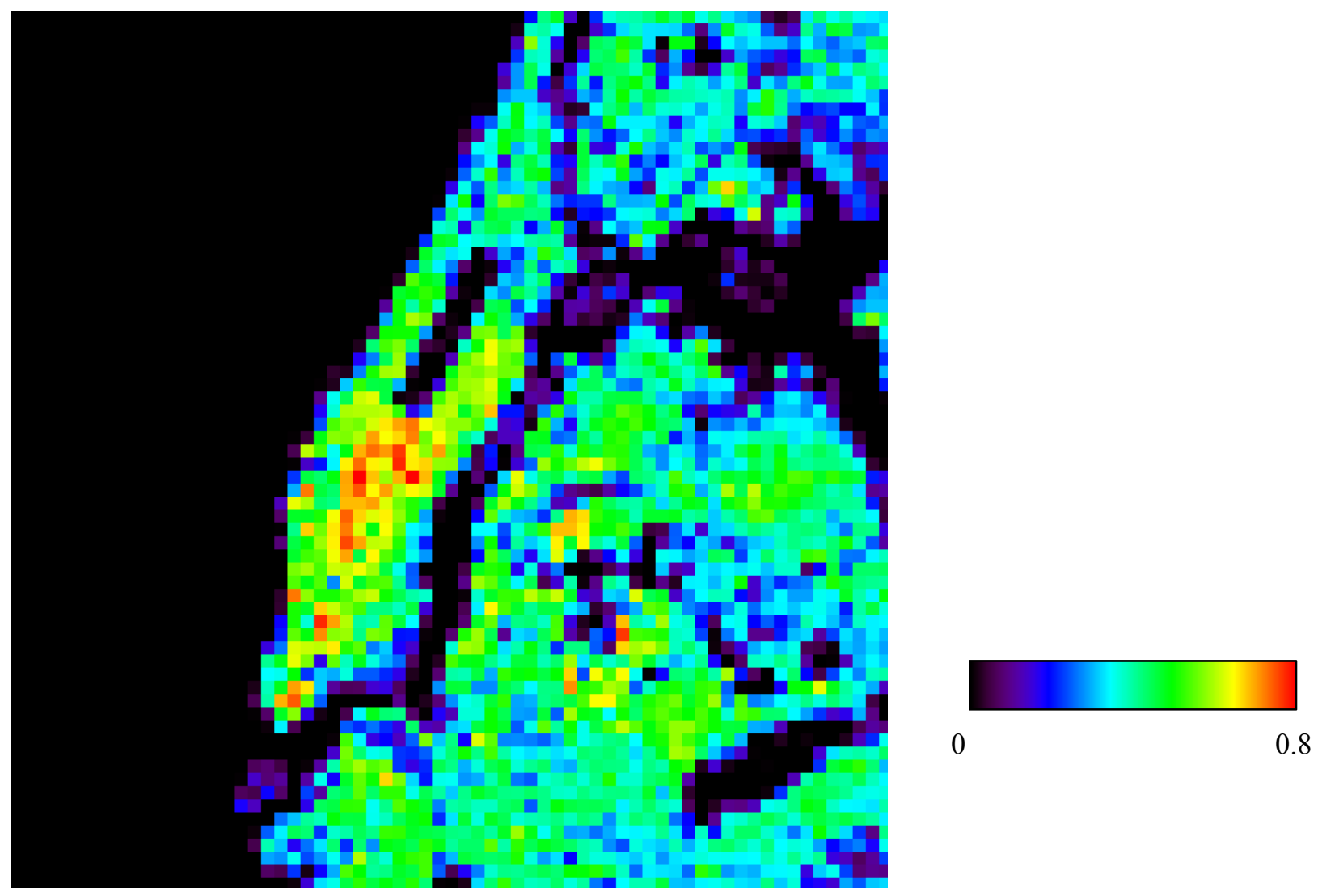

(e) 


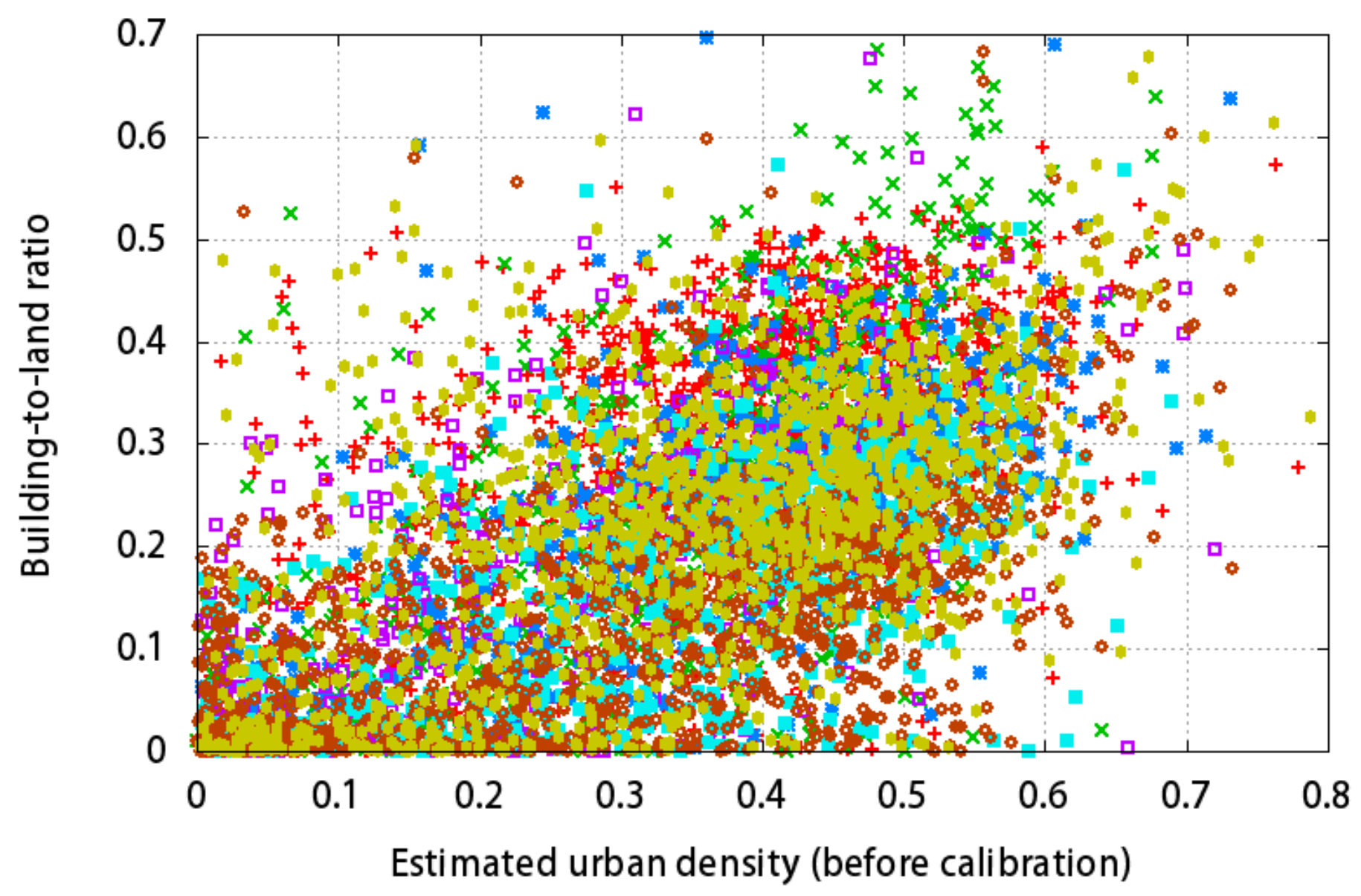

$\begin{array}{rrrrr}\text { Tokyo } & + & \text { Nagoya } & * & \text { Sendai } \\ \text { Kyoto } & \times & \text { Kobe } & \square & \text { Munich }\end{array}$

(a) 


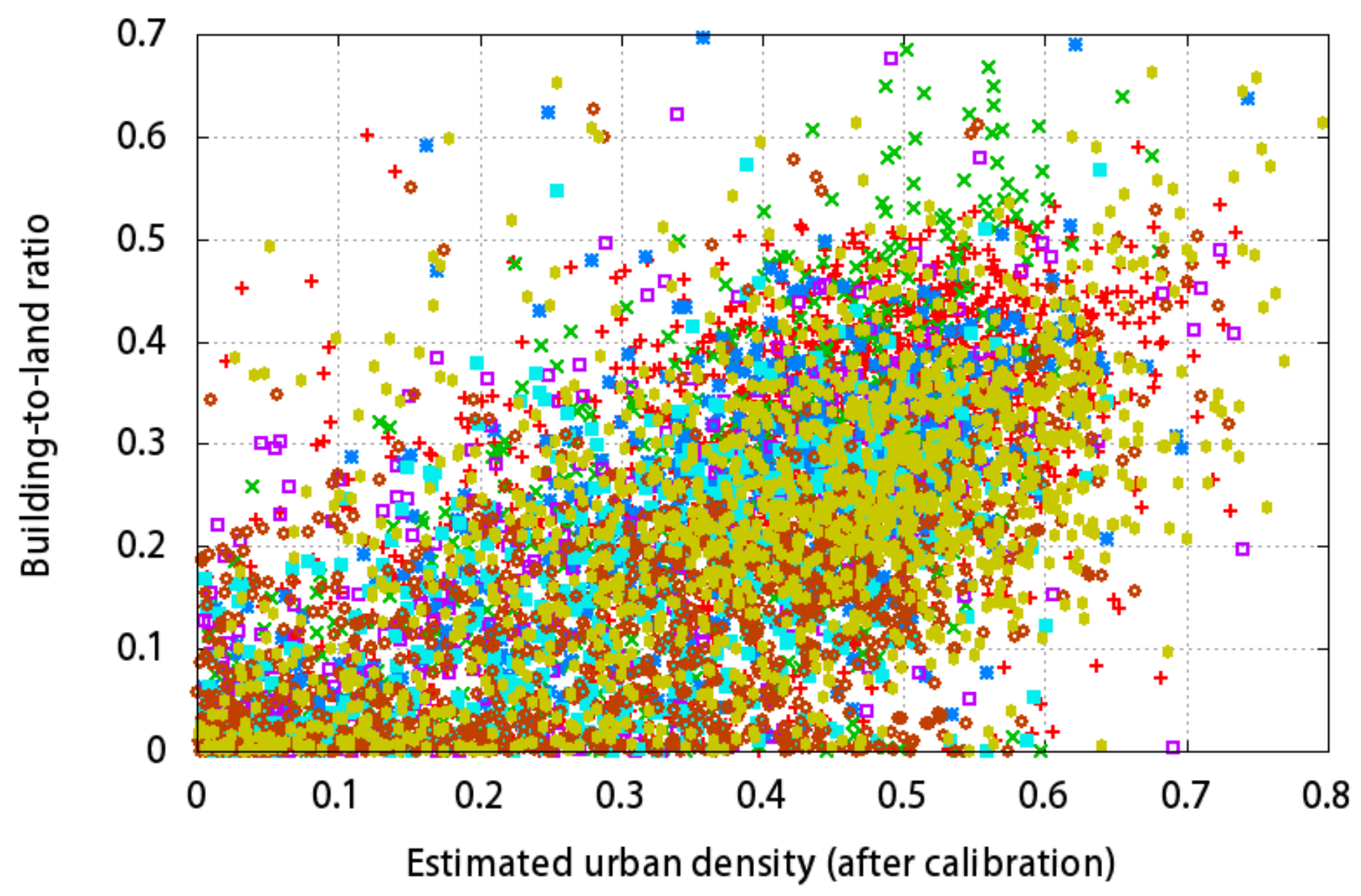

$\begin{array}{rrrrr}\text { Tokyo } & + & \text { Nagoya } & * & \text { Sendai } \\ \text { Kyoto } & \times & \text { Kobe } & \square & \text { Munich }\end{array}$

(b) 


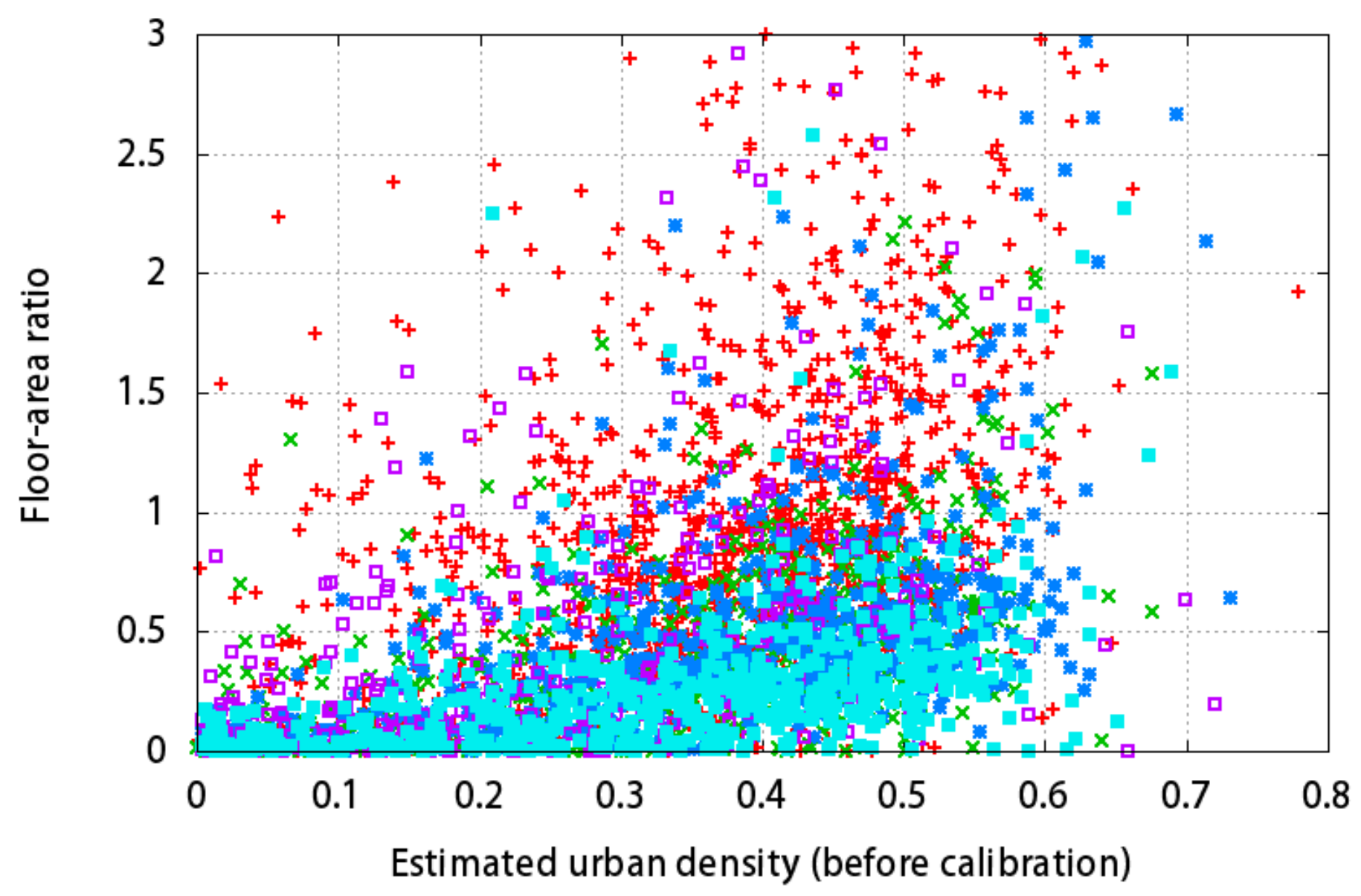

$\begin{array}{rrrrr}\text { Tokyo } & + & \text { Nagoya } & * & \text { Sendai } \\ \text { Kyoto } & \times & \text { Kobe } & \square\end{array}$

(a) 


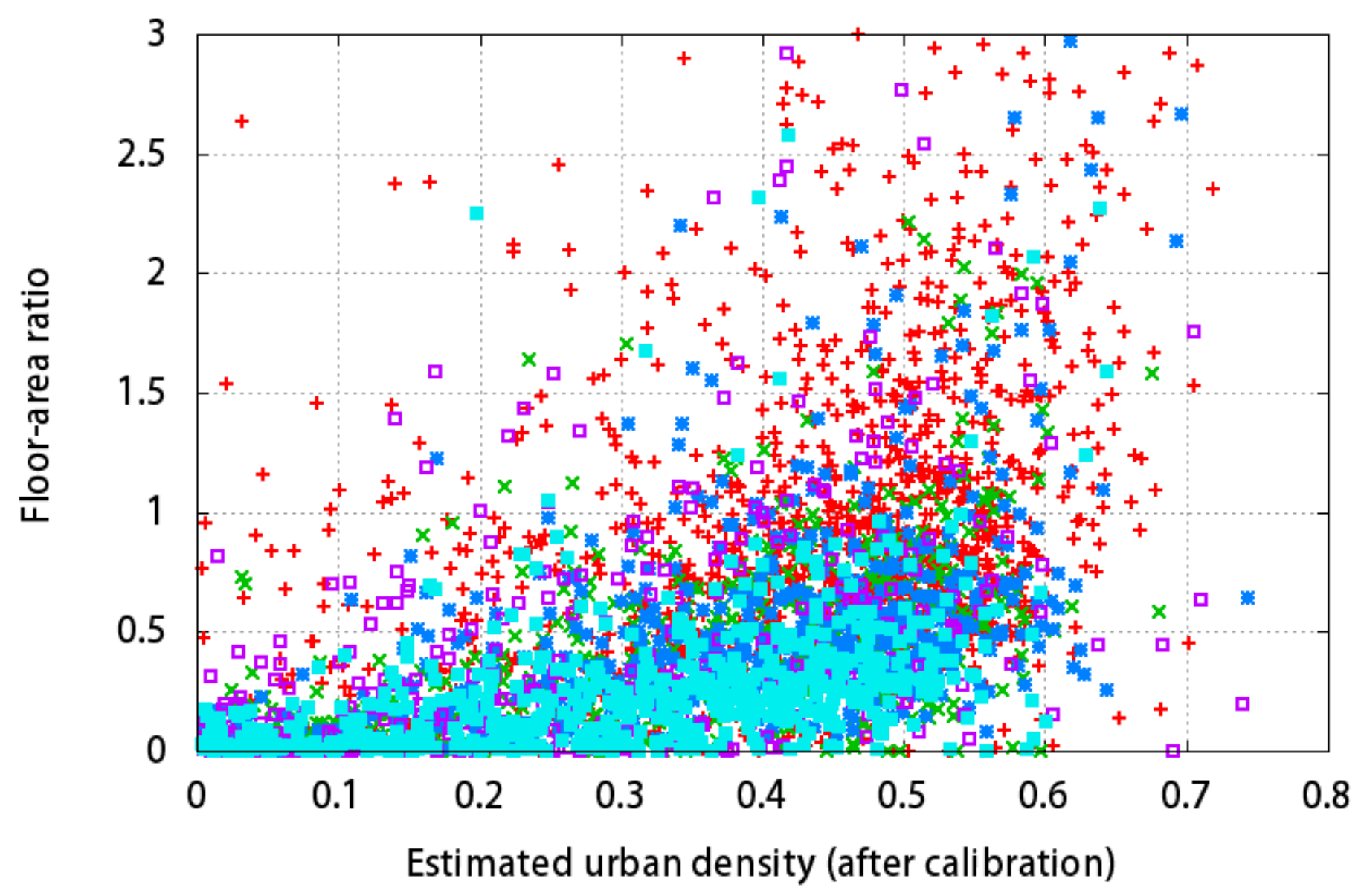

$\begin{array}{rrrrr}\text { Tokyo } & + & \text { Nagoya } & * & \text { Sendai } \\ \text { Kyoto } & \times & \text { Kobe } & \square\end{array}$

(b) 


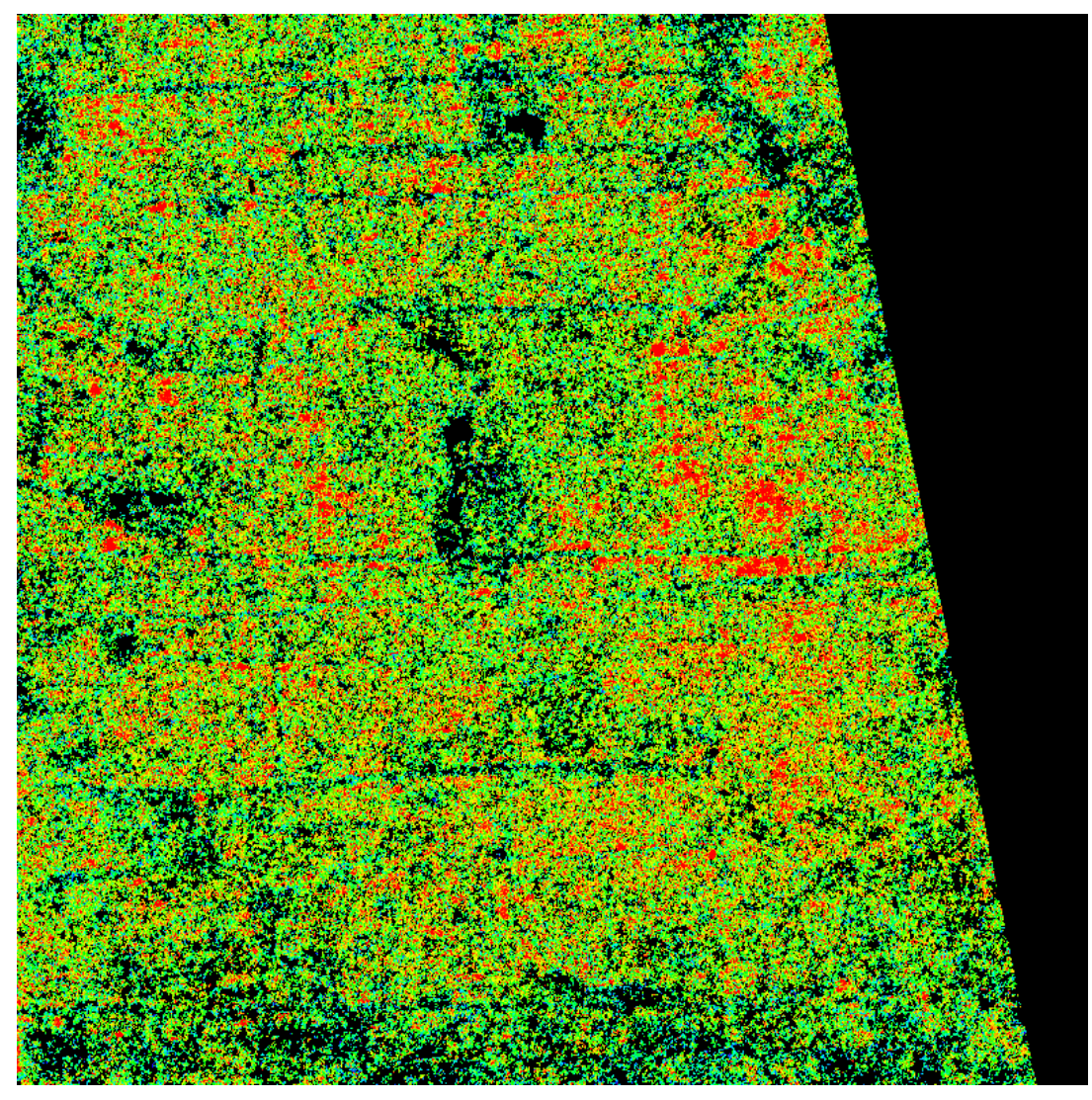

(a)

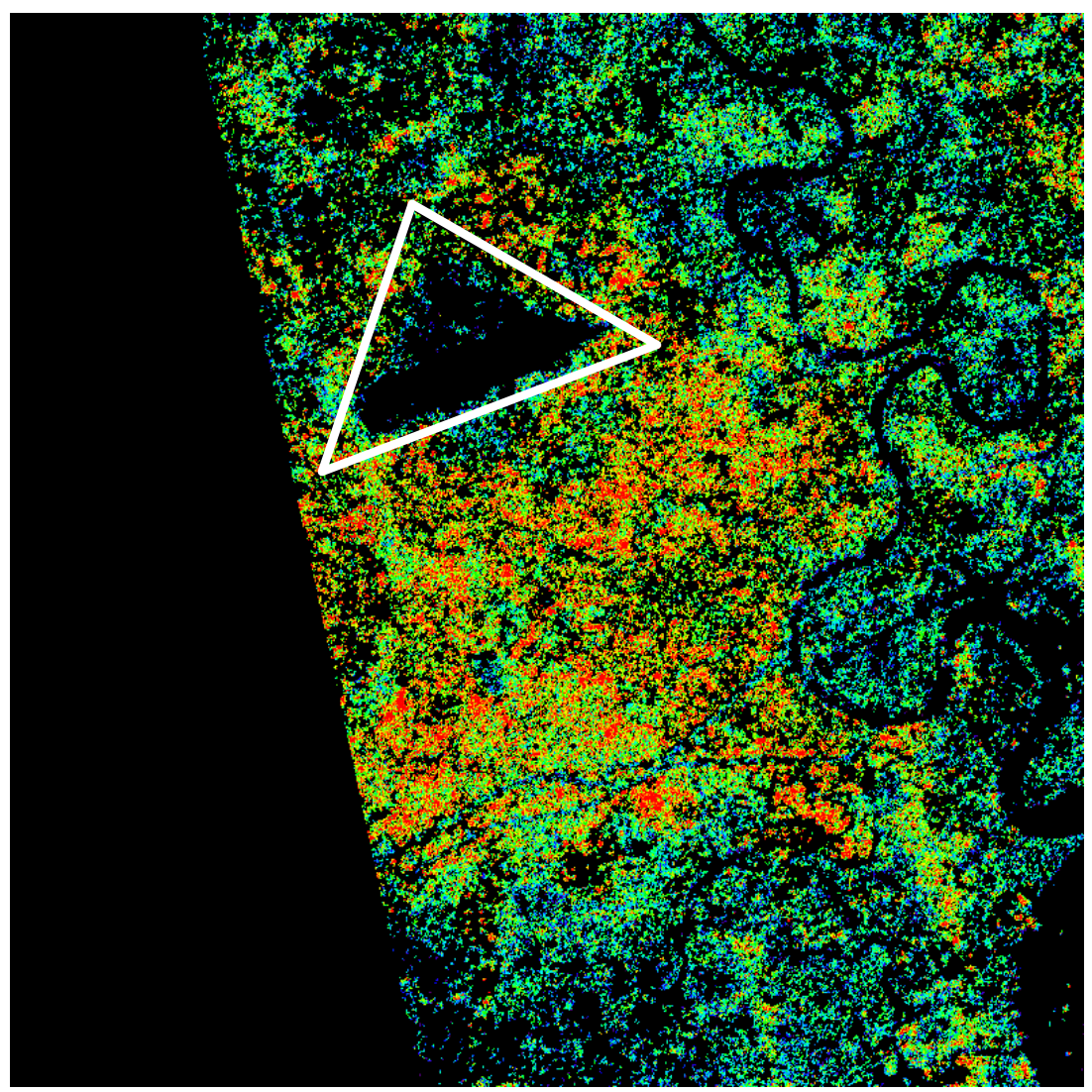

(b)
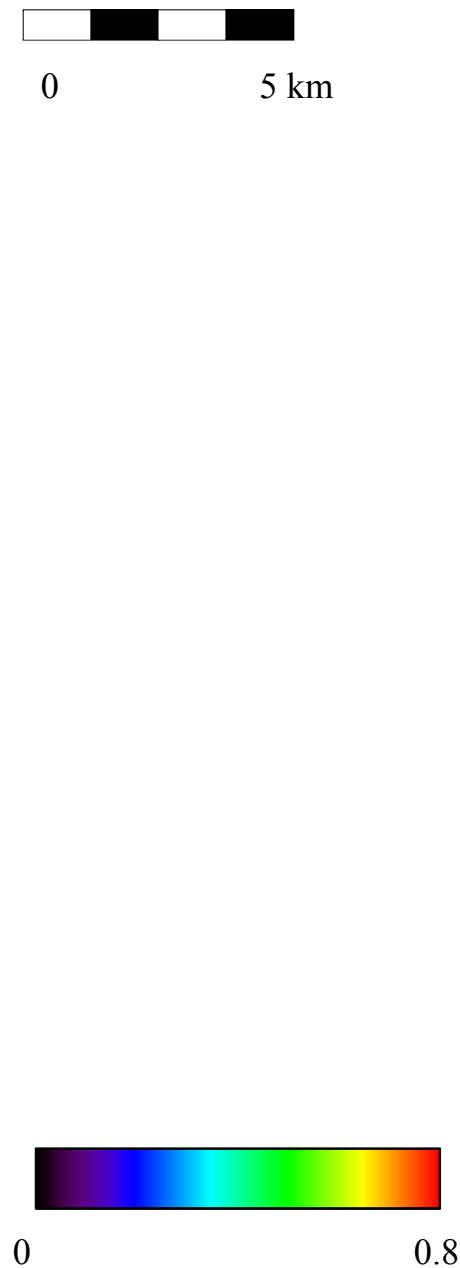


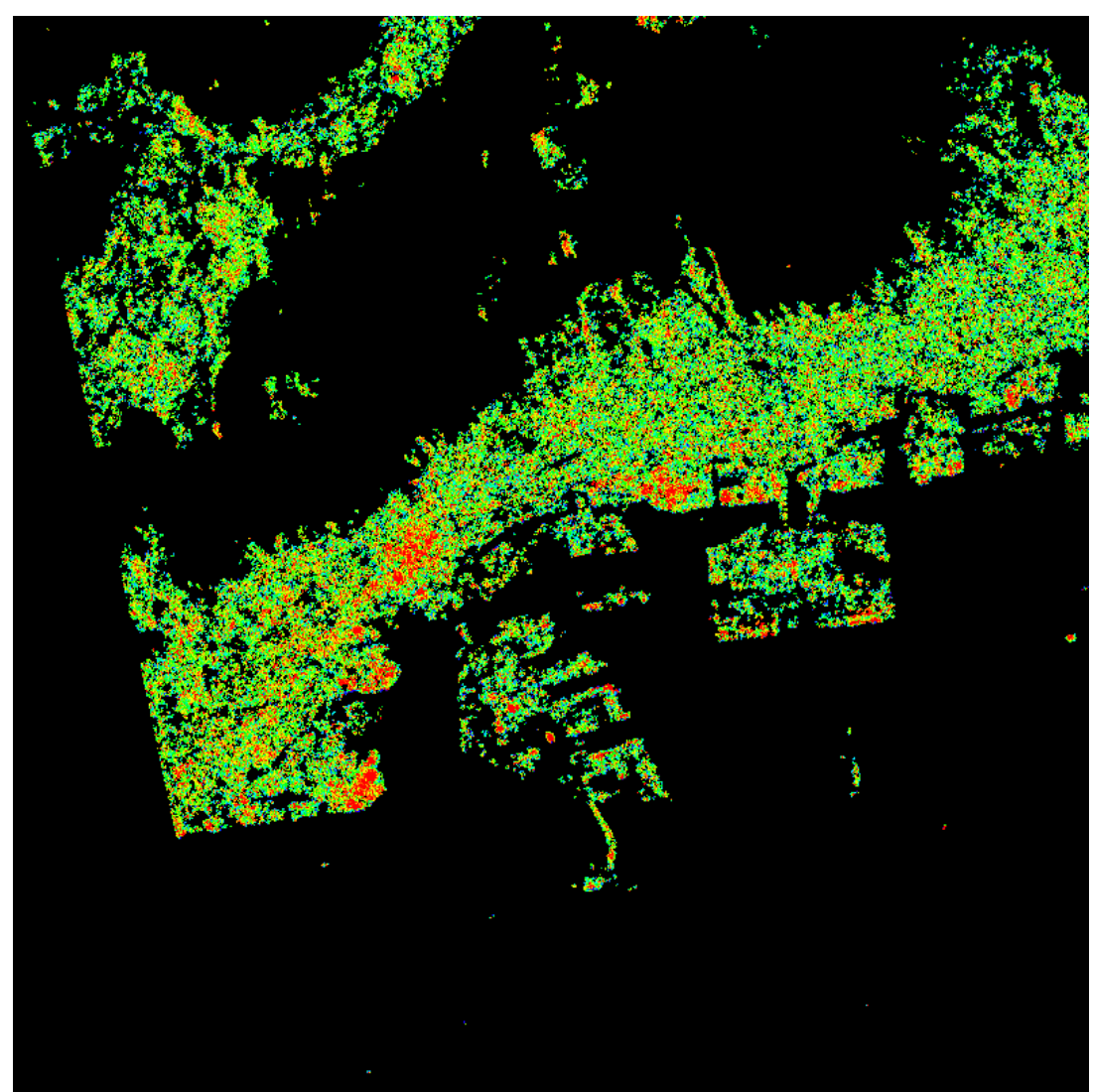

(c)

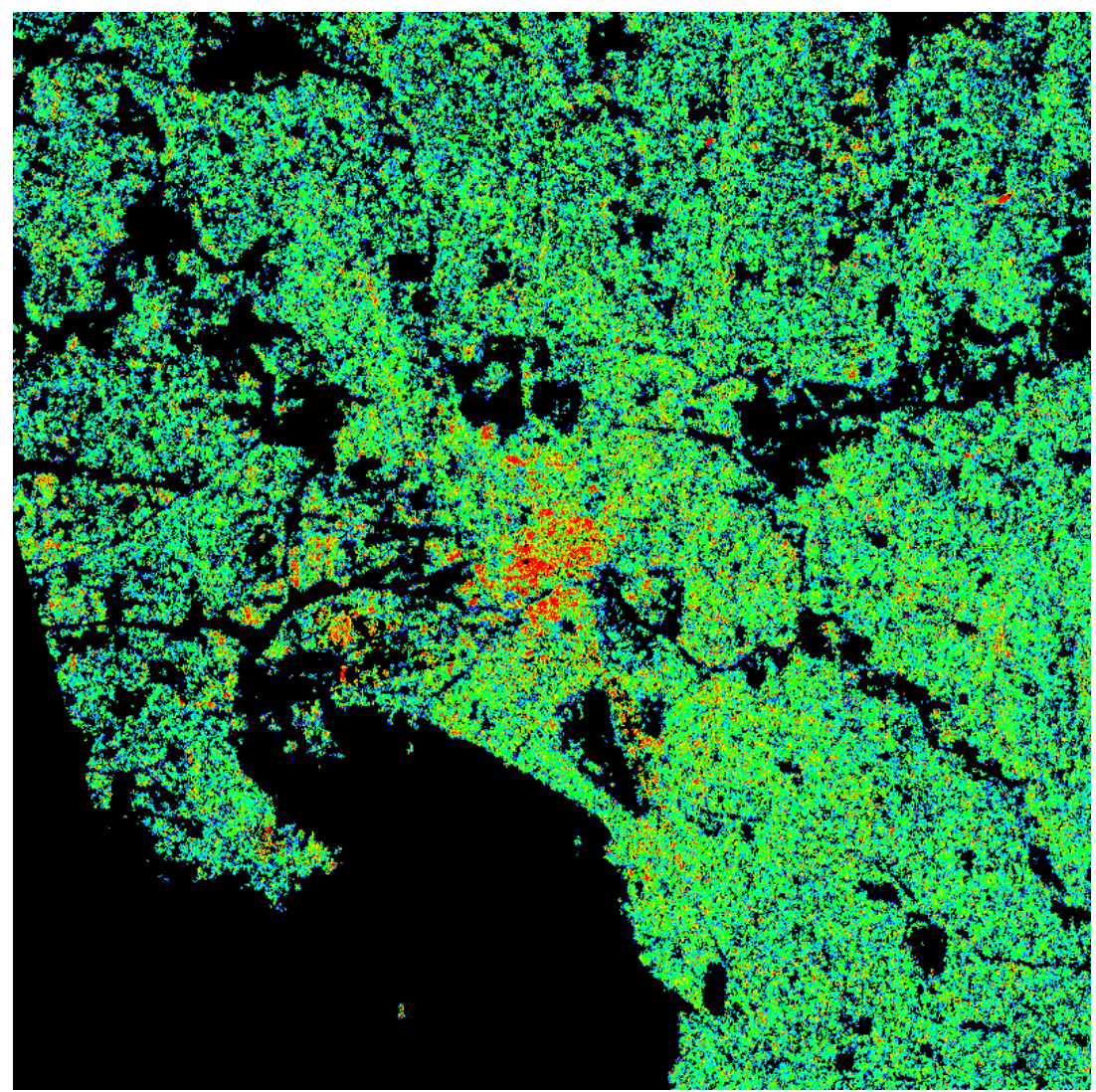

(d)
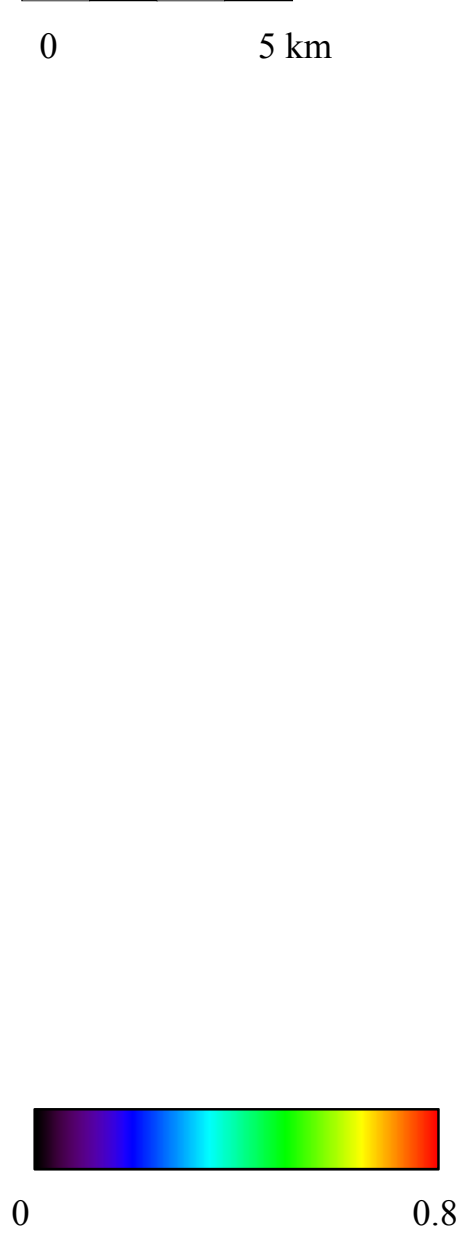


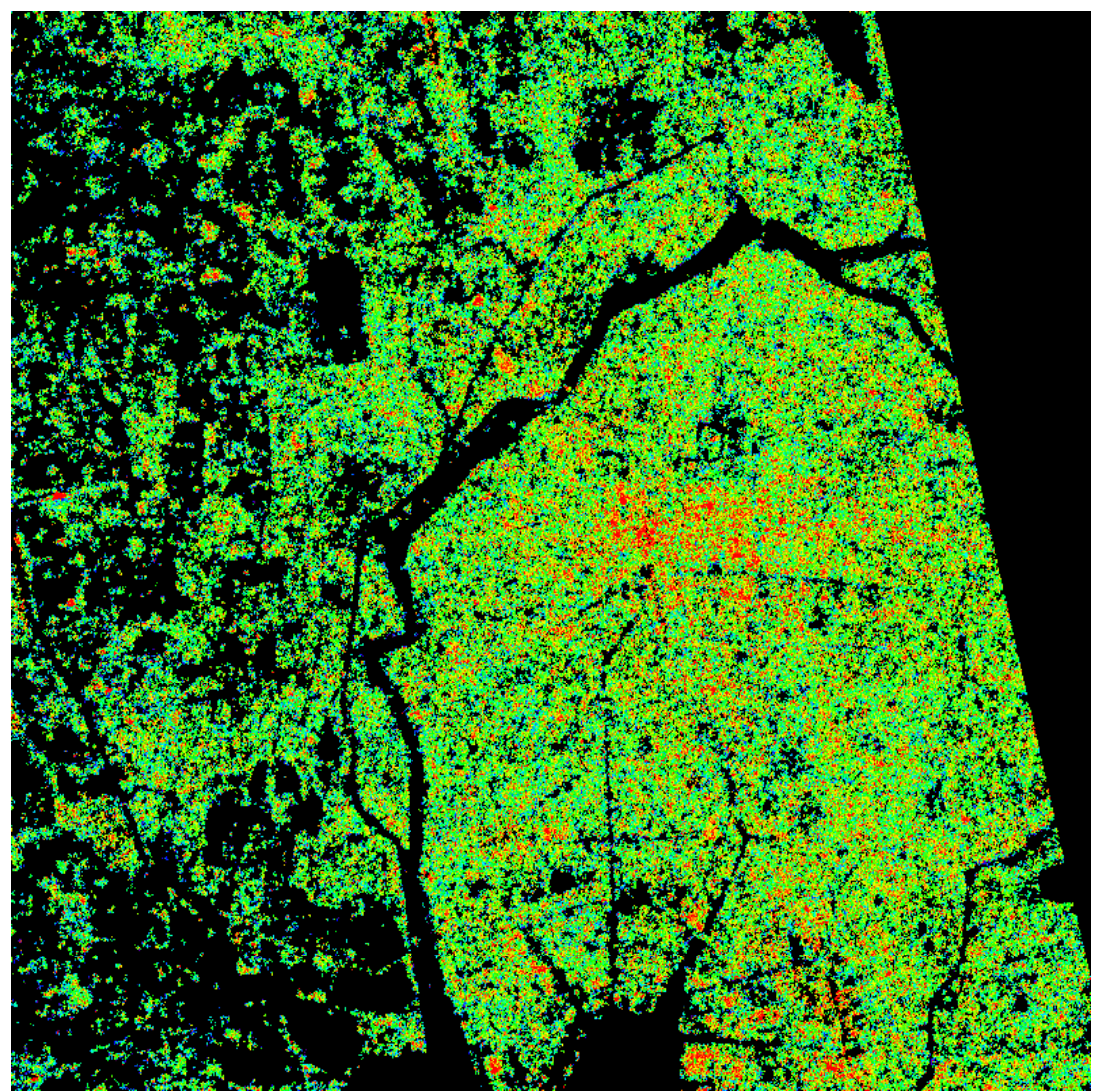

(e)

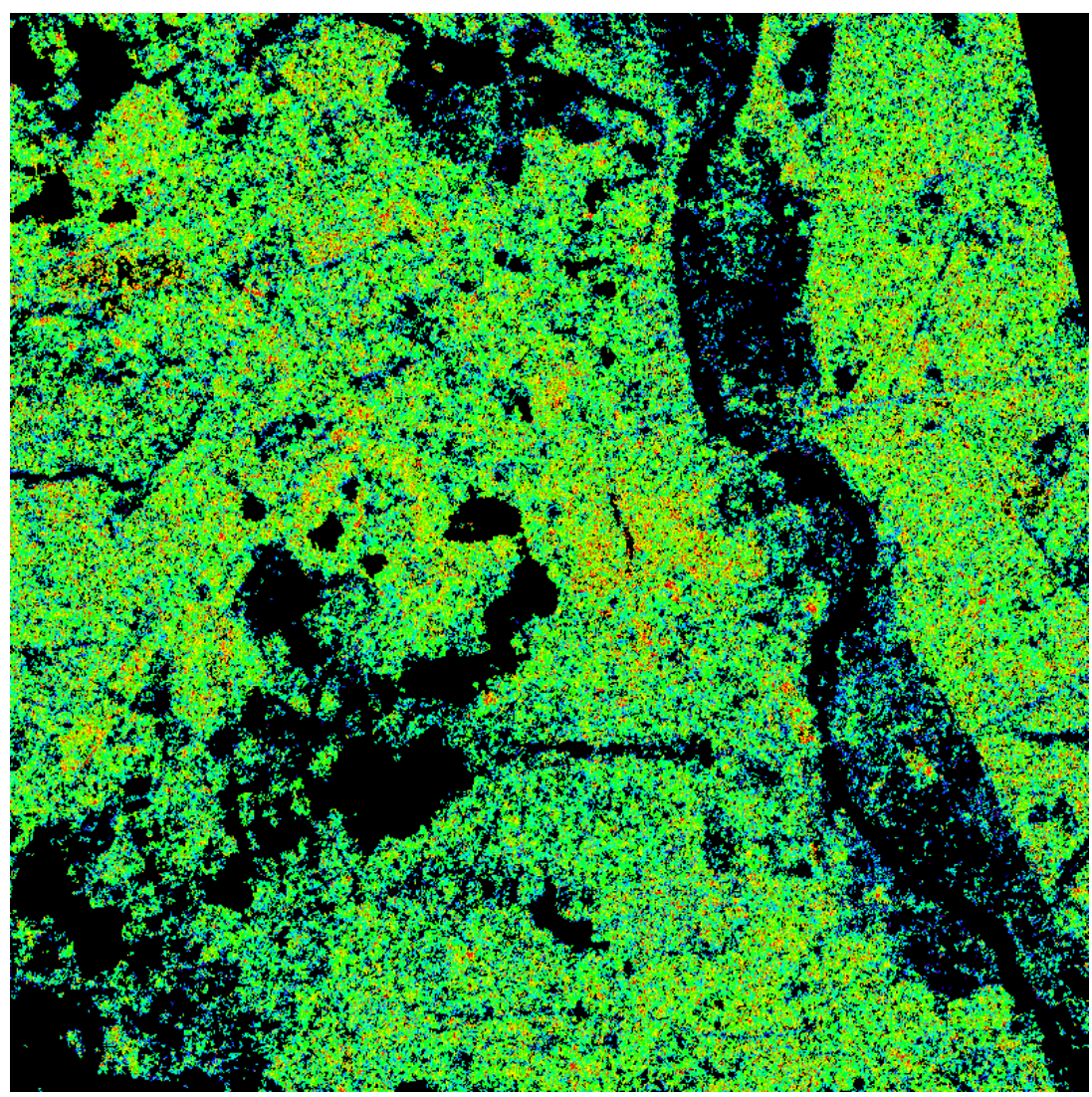

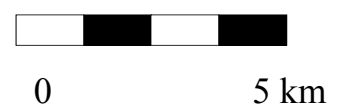

$5 \mathrm{~km}$

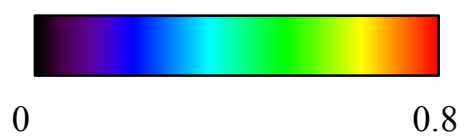

(f) 


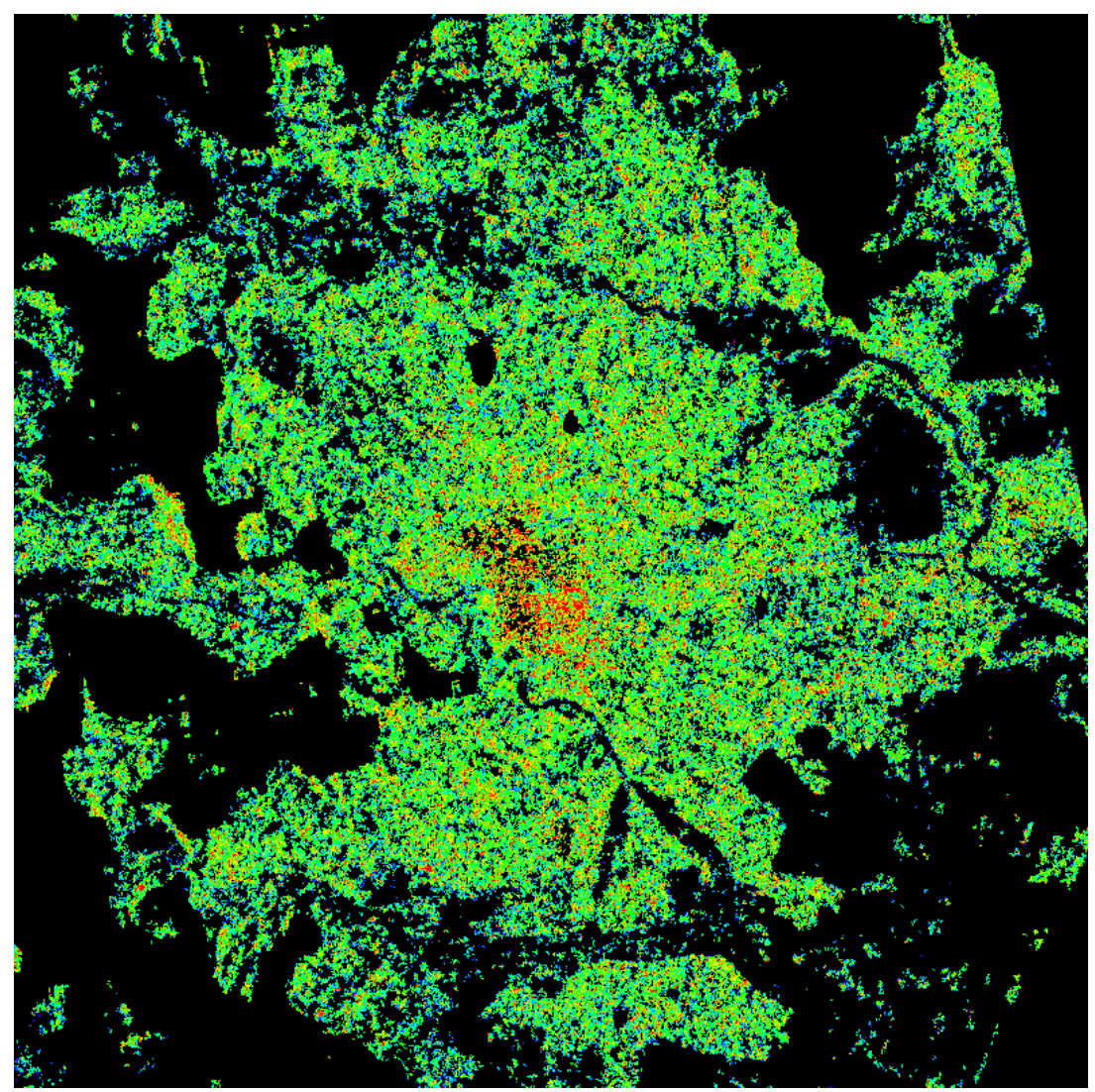

(g)
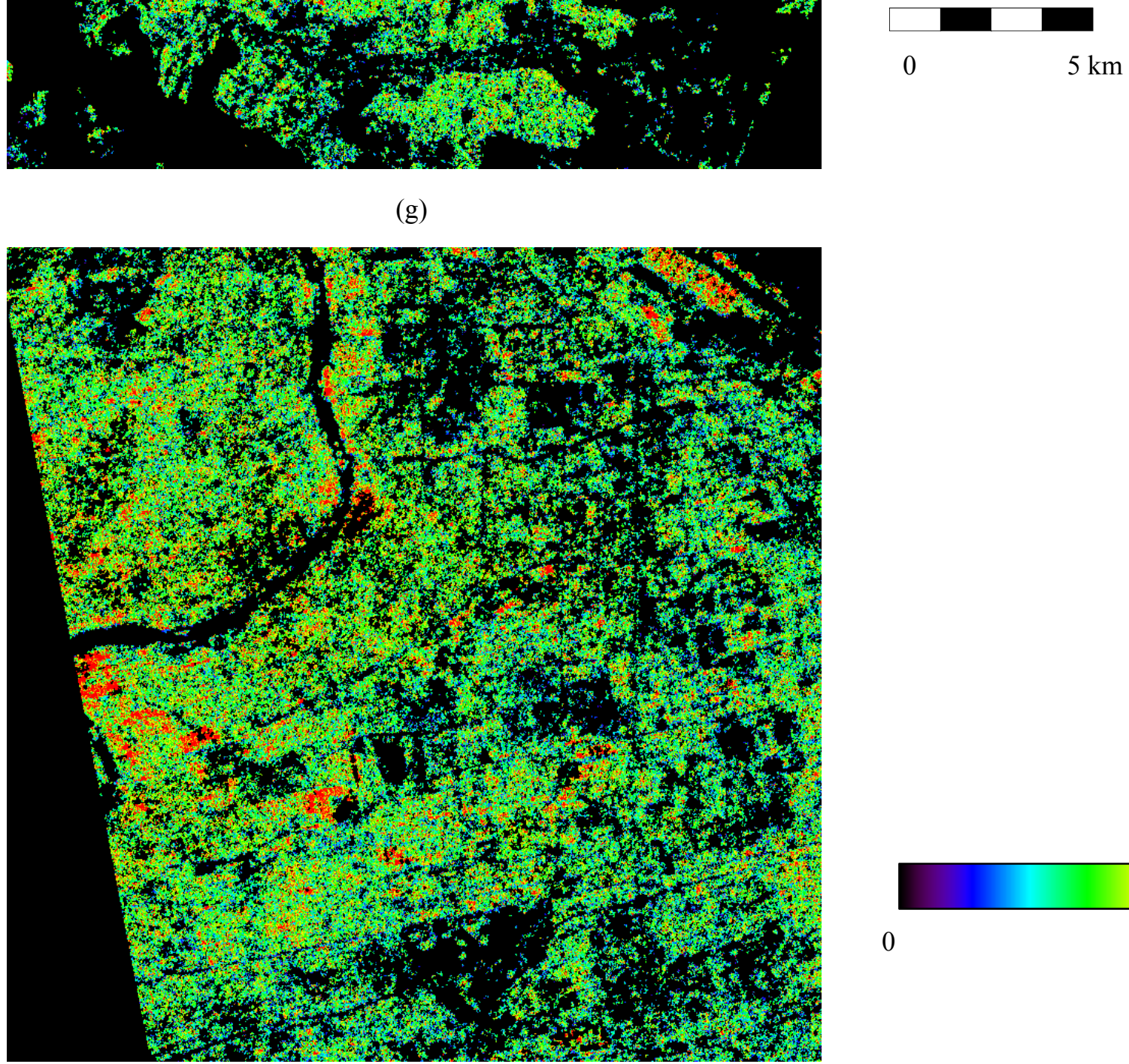

$5 \mathrm{~km}$

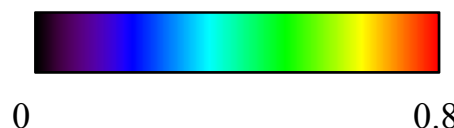

(h) 


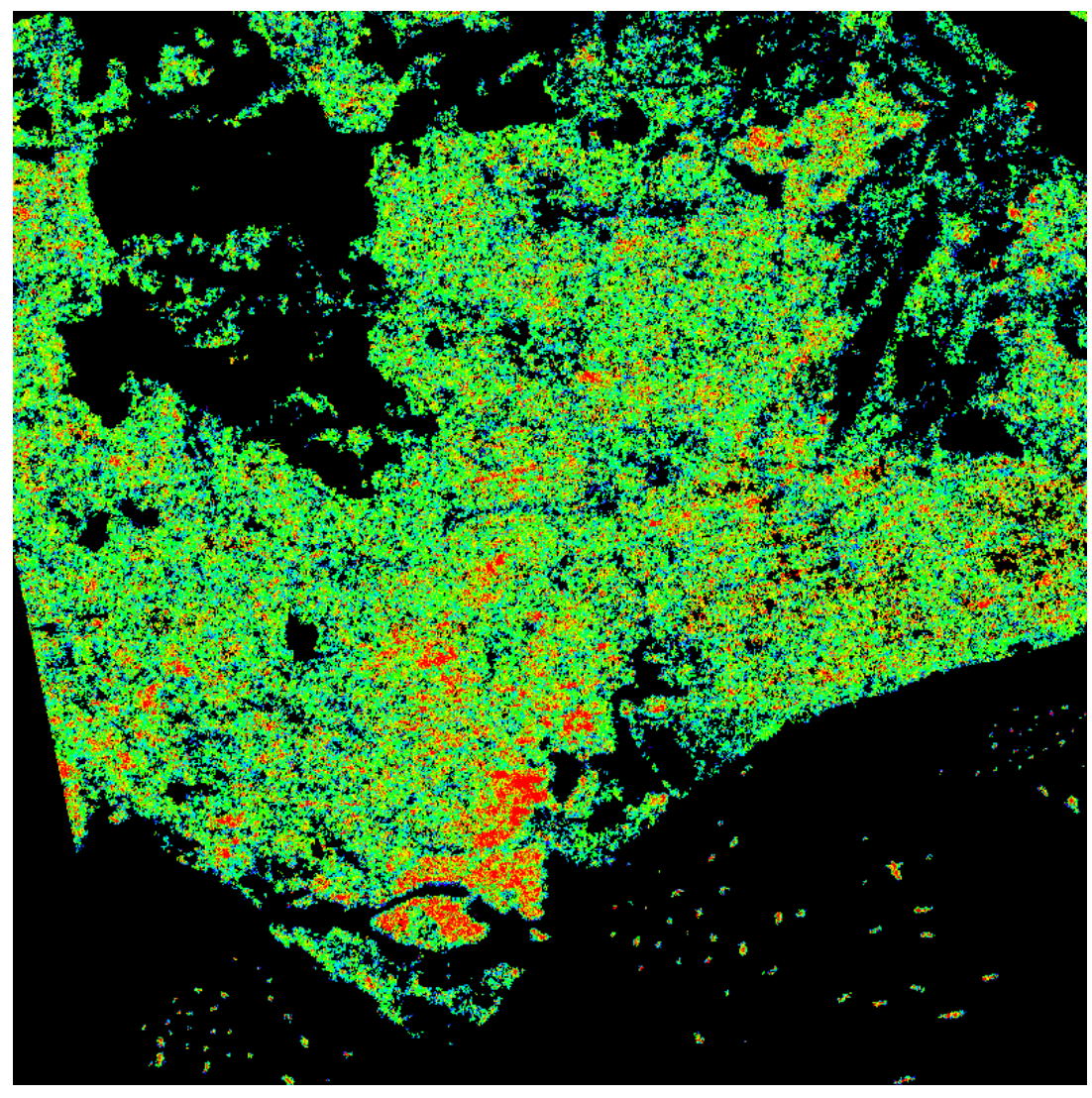

(i)

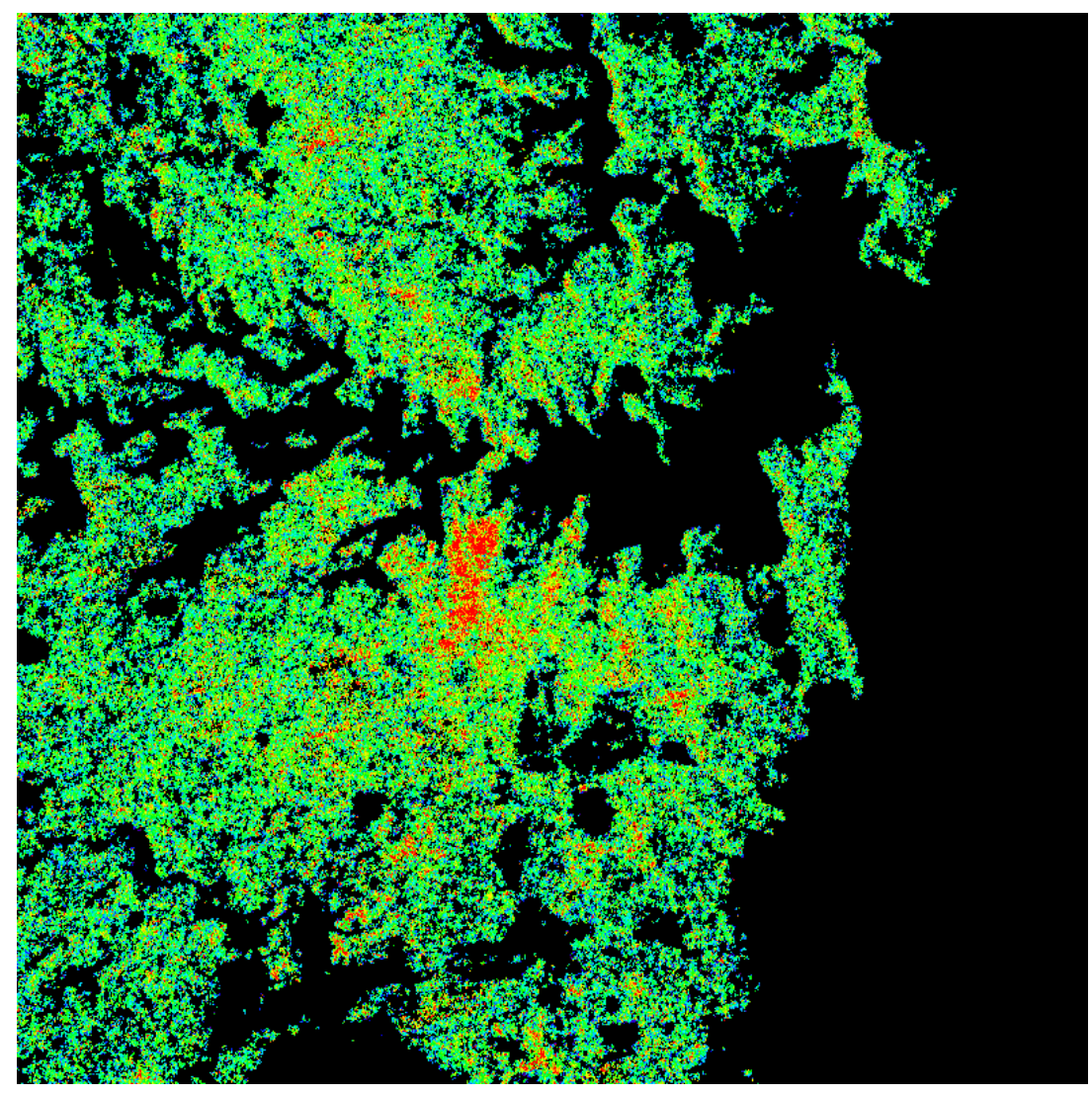

(j)
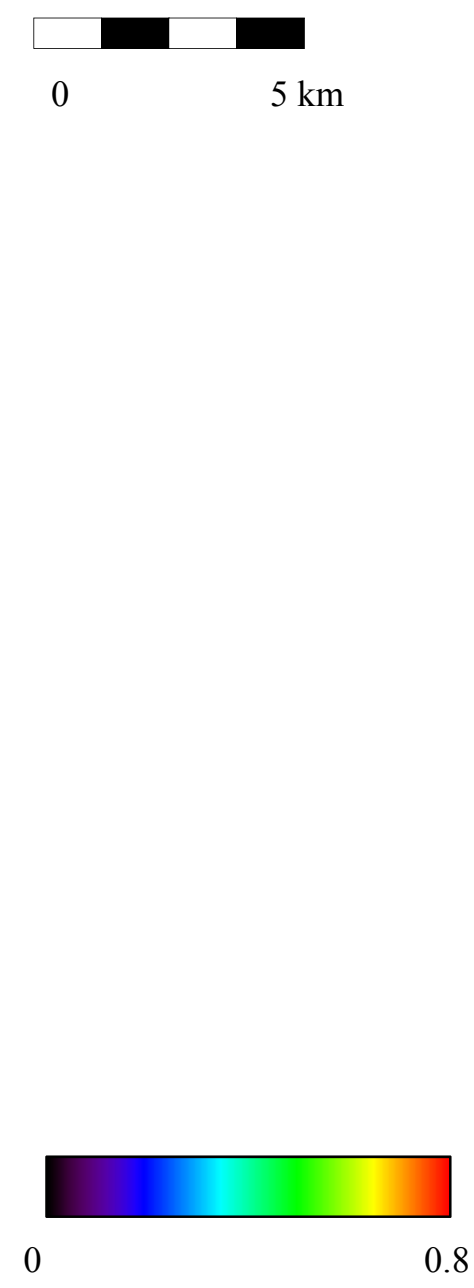

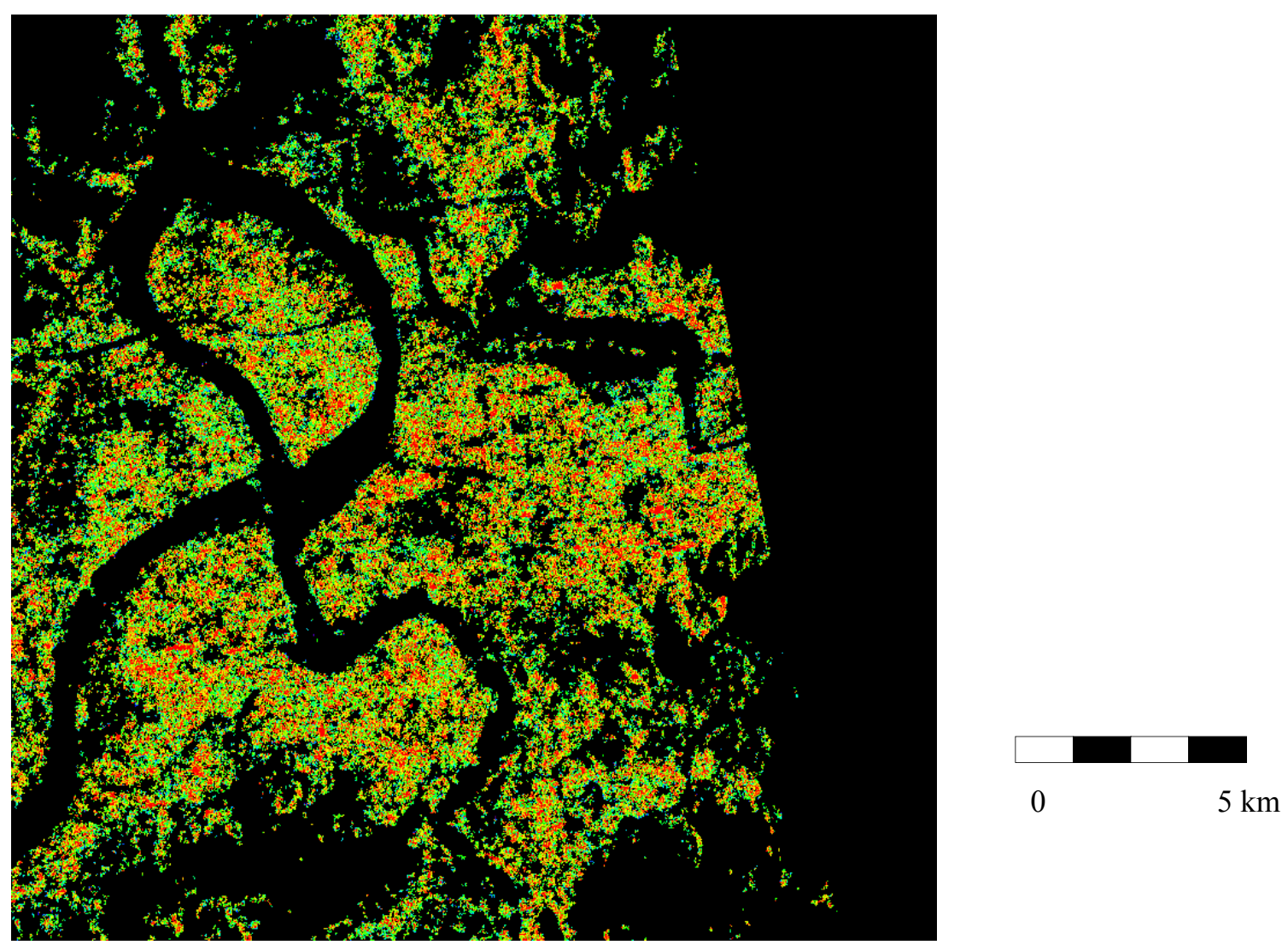

(k)

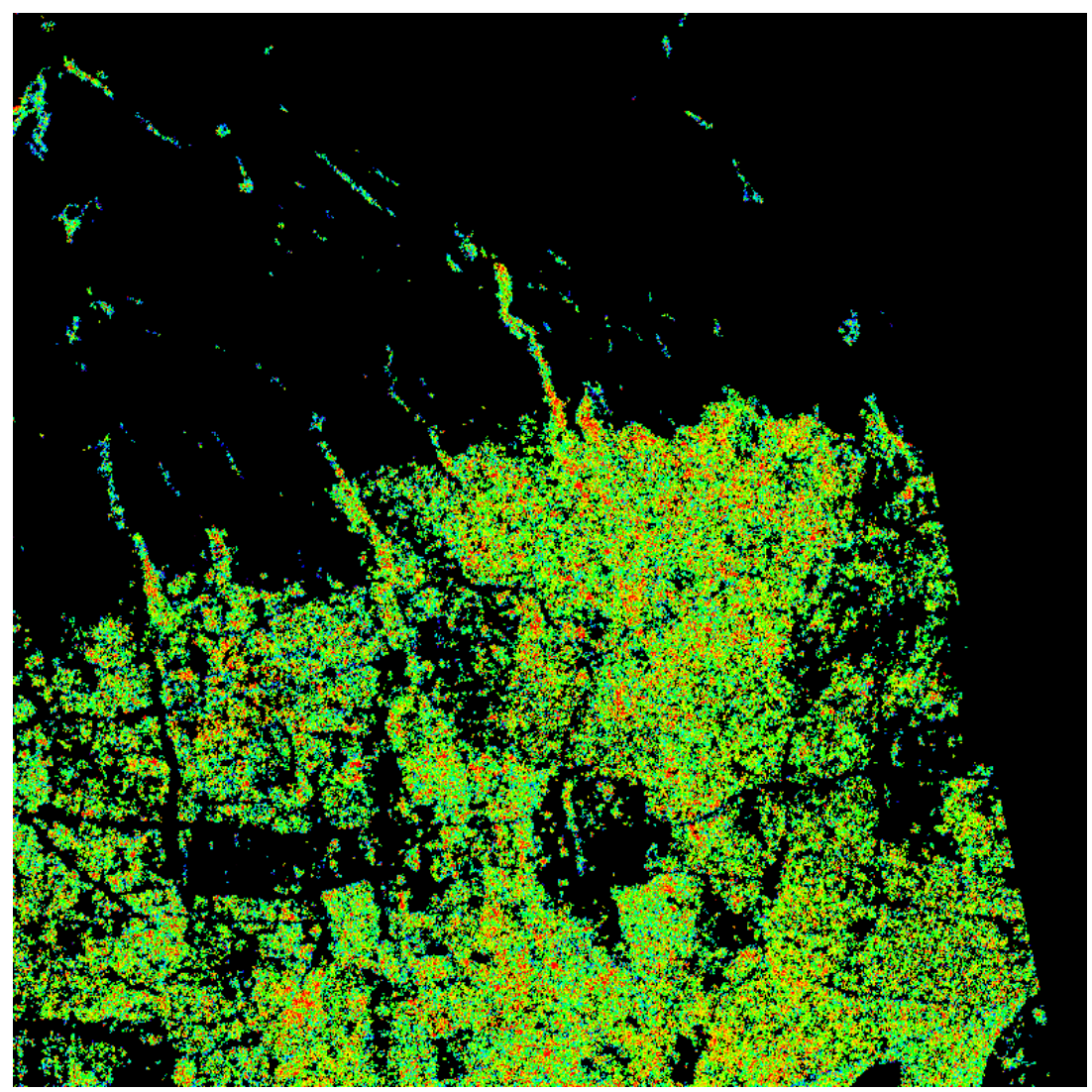

0

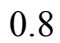

(1) 

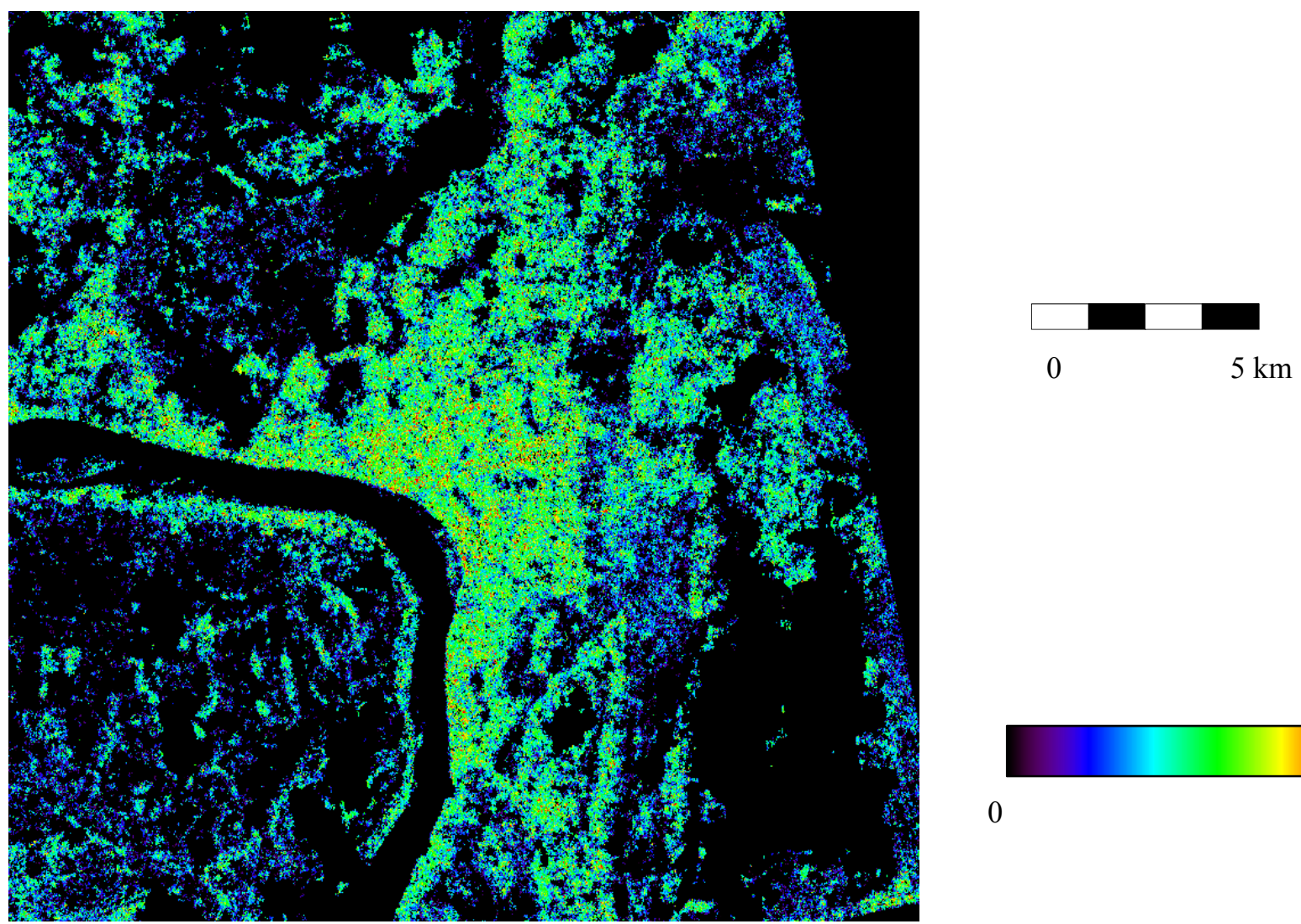

(m) 


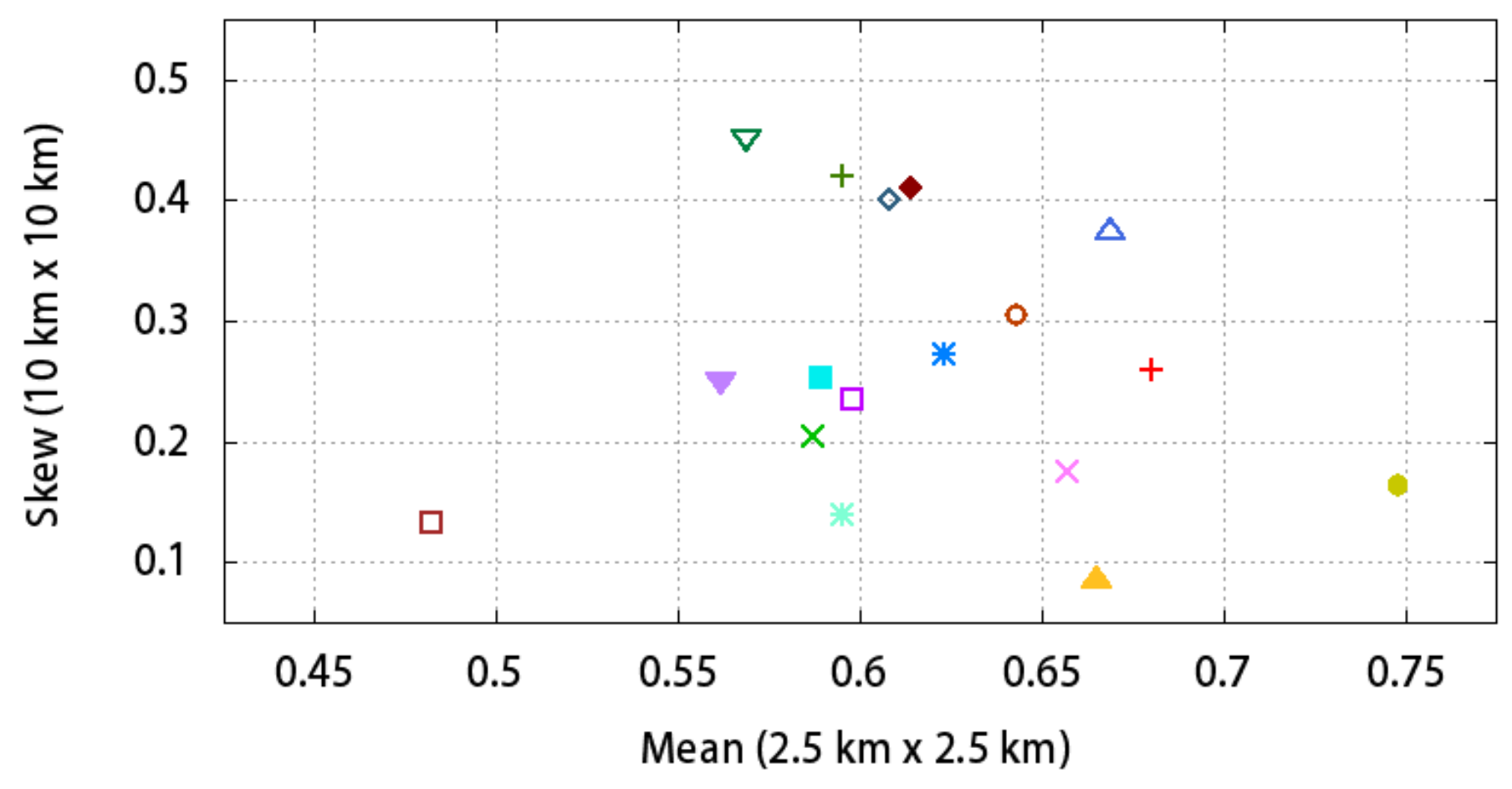

$\begin{array}{rrrr}\text { Tokyo } & + & \text { New York } & \\ \text { Kyoto } & \times & \text { Beijing } & \Delta \\ \text { Nagoya } & * & \text { Ho Chi Minh } & \Delta \\ \text { Kobe } & \square & \text { Melbourne } & \nabla \\ \text { Sendai } & \square & \text { New Delhi } & \nabla \\ \text { Munich } & \circ & \text { Shanghai } & \diamond\end{array}$

Singapore

Sydney +

Taipei

Tehran

Vientiane

(a) 


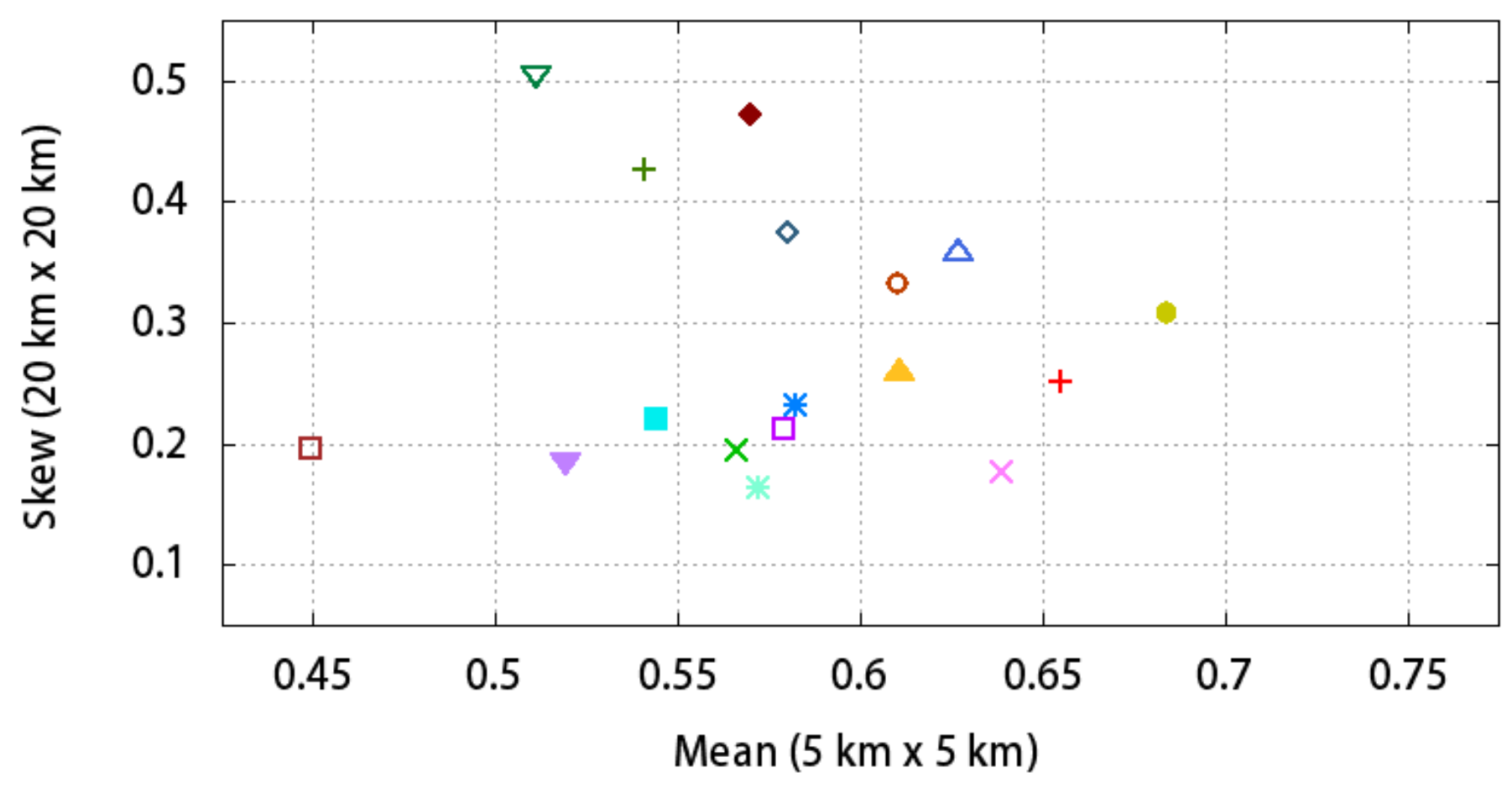

$\begin{array}{rrrr}\text { Tokyo } & + & \text { New York } & \\ \text { Kyoto } & \times & \text { Beijing } & \Delta \\ \text { Nagoya } & * & \text { Ho Chi Minh } & \Delta \\ \text { Kobe } & \square & \text { Melbourne } & \nabla \\ \text { Sendai } & \square & \text { New Delhi } & \nabla \\ \text { Munich } & \circ & \text { Shanghai } & \diamond\end{array}$

Singapore

Sydney +

Taipei

Tehran

Vientiane

(b) 